Archivio

\title{
d'Annunzio
}

Vol. 6

Ottobre 2019

2

Edizioni

Ca'Foscari

O

$\begin{array}{lllllllll}0 & 0 & 0 & 0 & 0 & 0 & 0 & 0\end{array}$

0

$10>0>0>0$

0

o

$0 \longdiv { 0 }$

$0>0$

0 o 0

0

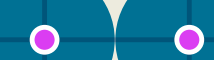

000

○

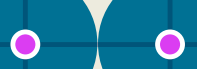

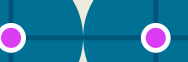

$0>0$

$0 \quad 0$

○

00

0

$0 \longdiv { 0 }$

10

$0 \quad 0$

0 o 0

00

0

ororo o o

○

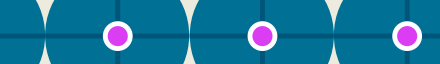

○

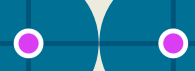

$0 \longdiv { 0 }$

0

10000

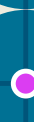
- $0<0$

0

$0>0$

$0 \longdiv { 2 }$

$0 \longdiv { 0 }$

C

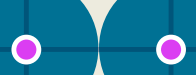

0 o 0

$0>0>0$

$0 \longdiv { 0 }$

00

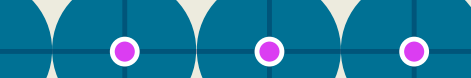

$\circ$

. 0

0

00 


\section{Archivio d'Annunzio}

Direttori

Ilaria Crotti

Pietro Gibellini

Paolo Puppa

Edizioni Ca' Foscari - Digital Publishing Università Ca' Foscari Venezia Dorsoduro 3246, 30123 Venezia

URL http://edizionicafoscari.unive.it/it/edizioni/riviste/annalidi-ca-foscari-serie-orientale/ 


\title{
Archivio d'Annunzio
}

\author{
Rivista annuale
}

Direttori Ilaria Crotti (Università Ca' Foscari Venezia, Italia) Pietro Gibellini (Università Ca' Foscari Venezia, Italia) Paolo Puppa (Università Ca’ Foscari Venezia, Italia)

Comitato scientifico Giuseppina Dal Canton (Università degli Studi di Padova, Italia) Adriana Guarnieri (Università Ca' Foscari Venezia, Italia) Mario Isnenghi (Università Ca' Foscari Venezia, Italia) Tiziana Piras (Università degli Studi di Trieste, Italia) Ricciarda Ricorda (Università Ca' Foscari Venezia, Italia) Alessandro Scarsella (Università Ca' Foscari Venezia, Italia)

Comitato di lettura Raffaella Bertazzoli (Università degli Studi di Verona, Italia) Maria Ida Biggi (Università Ca' Foscari Venezia, Italia) Milva Maria Cappellini (Centro Nazionale di Studi dannunziani, Pescara, Italia) Raffaella Castagnola (UniversitätZürich, Schweiz) Simona Costa (Università degli Studi Roma Tre, Italia) Luciano Curreri (Université de Liège, Belgique) Željko Đurić (Univerzitet u Beogradu, Srbija) Silvia Fabrizio-Costa (Université de Caen-Basse Normandie, France) Monica Giachino (Università Ca' Foscari Venezia, Italia) Maria Rosa Giacon (Centro Nazionale di Studi dannunziani, Pescara, Italia) François Livi (Université Paris-Sorbonne, France) Niva Lorenzini (Alma Mater Studiorum - Università di Bologna, Italia) Paola Martinuzzi (Università Ca' Foscari Venezia, Italia) Gianni Oliva (Università degli Studi “G. D’Annunzio" Chieti-Pescara, Italia) Lucia Re (University of California Los Angeles, USA) Michela Rusi (Università Ca' Foscari Venezia, Italia) Silvana Sinisi (Università degli Studi di Salerno, Italia)

Comitato di redazione Roberta Favia (Università Ca' Foscari Venezia, Italia) Beniamino Mirisola (Università Ca' Foscari Venezia, Italia) Maddalena Rasera (Università degli Studi di Verona, Italia) Elena Sbrojavacca (Università Ca' Foscari Venezia, Italia) Francesca Suppa (Università Ca' Foscari Venezia, Italia) Veronica Tabaglio (Università Ca' Foscari Venezia, Italia) Alessandra Trevisan (Università Ca' Foscari Venezia, Italia) Silvia Uroda (Università Ca' Foscari Venezia, Italia) Alberto Zava (Università Ca' Foscari Venezia, Italia) (segretario) Giulia Zava (Università Ca' Foscari Venezia, Italia)

Direttore Responsabile Michela Rusi (Università Ca' Foscari Venezia, Italia)

\section{Direzione e redazione}

Università Ca' Foscari Venezia, Dipartimento di Studi Umanistici

Dorsoduro 3246, 30123 Venezia, Italia

Editore Edizioni Ca' Foscari - Digital Publishing | Dorsoduro 3246, 30123 Venezia, Italia| ecf@unive.it

Stampa Logo srl, via Marco Polo 8, 35010 Bogoricco (PD)

C 2019 Università Ca' Foscari Venezia

(c) 2019 Edizioni Ca' Foscari - Digital Publishing per la presente edizione

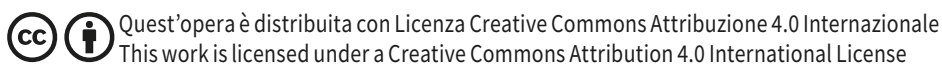

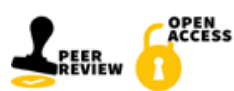

Certificazione scientifica delle Opere pubblicate da Edizioni Ca' Foscari - Digital Publishing: tutti i saggi pubblicati hanno ottenuto il parere favorevole da parte di valutatori esperti della materia, attraverso un processo di revisione anonima sotto la responsabilità del Comitato scientifico della collana. La valutazione è stata condotta in aderenza ai criteri scientifici ed editoriali di Edizioni Ca' Foscari. Scientific certification of the works published by Edizioni Ca' Foscari - Digital Publishing: all essays published in this volume have received a favourable opinion by subject-matter experts, through an anonymous peer review process under the responsibility of the Scientific Committee of the series. The evaluations were conducted in adherence to the scientific and editorial criteria established by Edizioni Ca' Foscari.

Qualunque parte di questa pubblicazione può essere riprodotta, memorizzata in un sistema di recupero dati o trasmessa in qualsiasi forma o con qualsiasi mezzo, elettronico o meccanico, senza autorizzazione, a condizione che se ne citi la fonte.

Any part of this publication may be reproduced, stored in a retrieval system, or transmitted in any form or by any means without 


\section{Sommario}

OFFICINA DANNUNZIANA

La forma breve in d'Annunzio

Introduzione

Ilaria Crotti

«Vogliatemi bene»: lettere di Matilde Serao a Gabriele d'Annunzio

Alessandra Trevisan

Ferrara, Pisa, Ravenna: le «città soavi»

Cristina Montagnani

Dignità del dolore

Esercizi di consapevolezza sul Notturno di Gabriele d'Annunzio

Federica Maria Giallombardo

La memoria 'breve' delle Faville del maglio

Raffaella Bertazzoli

CIVILTÀ DANNUNZIANA

L'eredità di Ovidio in Giovanni Pascoli

e Gabriele d'Annunzio

Maria Belponer

Contro i nuovi Barbari: d'Annunzio, Spengler,

l'ombra di Cesare

Alfredo Sgroi

Stato, problemi, applicazioni critiche

della filologia dannunziana

Elena Maiolini 
CIVILTÀ DANNUNZIANA

Cristina Montagnani, Pierandrea De Lorenzo

Come lavorava d'Annunzio

Elena Maiolini

Cecilia Gibellini

La miglior parte della mia anima. Lettere alla moglie (1883-1893)

Paolo Parachini

Maurizio Serra

D’Annunzio le Magnifique

Veronica Tabaglio 


\section{Introduzione}

\section{Ilaria Crotti}

Il presente fascicolo di Archivio d'Annunzio, il sesto di una serie che è venuta acquisendo negli anni un profilo di tutto rilievo, nell'alternare a fascicoli dedicati a disamine di tenore comparatistico, storiografico ed estetico altri riservati in misura maggiore a interpretazioni testuali e a verifiche filologiche, interroga una delle forme che contrassegna in misura rilevante la produzione letteraria italiana, e fin dalle sue origini, ossia quella distintasi per brevitas: una scelta che chiama in causa anche sinonimi e antonimi implicati nella forma 'breve' e i significati non univoci che essa veicola.

Ecco che nella sezione Officina dannunziana vari apporti si cimentano proprio in detto ambito, che si rivela estremamente proficuo per sondare non solo il laboratorio ideativo dell'Imaginifico ma anche le relazioni, sia testuali-formali sia biografiche e autobiografiche, poste in essere.

Così, il contributo di Alessandra Trevisan, editando e commentando alcuni pezzi epistolari indirizzati da Matilde Serao a d'Annunzio, ne enuclea i risvolti autobiografici più significativi, atti ad appurare altresì determinate ipotesi interpretative, concernenti le prassi creative saggiate. Cristina Montagnani, da parte sua, conduce una fine lettura filologica intertestuale, non dimentica dei taccuini, del cantiere che il compositore delle Laudi pone in essere, mentre focalizza in particolare Ferrara, Pisa, Ravenna, le future Città del silenzio. Mentre è la prosa del Notturno la tipologia testuale presa in esame, soprattutto in accezione autobiografica, da Federica Maria Giallombardo, sono le Faville del maglio e la linea ermeneutica che le induce a dialogare a distanza ravvicinata con Alcyone il campo d'indagine prescelto da Raffaella Bertazzoli, vagliato con acribia sia filologica che interpretativa.

Grazie alla sezione Civiltà dannunziana il presente fascicolo si impreziosisce di apporti ulteriori. Come quello che si deve a Maria Belponer, la quale nelle sue pagine ha saputo cogliere con sagacia il dialogo incessante che ricorre tra il linguaggio del mito classico ovidiano nel Pascoli dei Poemi conviviali, dei quali Belponer nel 2009 è stata attenta curatrice, per un verso, e nel d'Annunzio dell'Alcyone, per un altro. 
Alfredo Sgroi, da parte sua, esplora con acribia la linea ideologicoestetica che intercorre tra il pescarese e Oswald Spengler, nell'attesa messianica di una figura utopica di uomo d'ordine - un novello Cesare cui spetterebbe il compito di risollevare le sorti della civiltà occidentale, segnate dal drastico declino di ideali intellettuali aristocratici, ritenute pertanto al crepuscolo. Infine, si deve al contributo di Elena Maiolini la formulazione di un bilancio estremamente calibrato degli studi più significativi dedicati alla filologia dannunziana: vera e propria rassegna utile ad arricchire il dibattito più avvertito, pronta com'è a puntualizzarne alcuni snodi metodologici.

A compimento del fascicolo, come di consueto, ecco lo spazio riservato alle recensioni, la prima delle quali destinata da Maiolini al recente volume di Cristina Montagnani e Pierandrea De Lorenzo, Come lavorava d'Annunzio (2018), mentre la seconda da Paolo Parachini a una selezione, attentamente curata da Cecilia Gibellini, delle missive inedite inviate dal consorte a Maria Hardouin, la 'principessa di Montenevoso': Lettere di d'Annunzio alla moglie (2018); in chiusura, il ponderoso ritratto dannunziano ad opera di Maurizio Serra in D'Annunzio le Magnifique, nella recensione di Veronica Tabaglio. 


\section{Officina dannunziana}

La forma breve in d'Annunzio 



\title{
"Vogliatemi bene»: lettere di Matilde Serao a Gabriele d'Annunzio
}

Alessandra Trevisan

Università Ca' Foscari Venezia, Italia

\begin{abstract}
Between ca. 1885 and 1927, Gabriele d'Annunzio and Matilde Serao became friends and contributed to journals in Rome and Naples; they met authors in Europe (especially in France), exchanged opinions about their works and also about their private life. She has been a vibrant constant on Vate's experience: she gave him advices and protected his affair with Eleonora Duse, who was also Matilde's friend. Serao's collection of letters, kept by Fondazione II Vittoriale degli Italiani in Gardone Riviera, explains some contours of a crucial friendship.
\end{abstract}

Keywords Lettere. Gabriele d’Annunzio. Matilde Serao. Eleonora Duse. Georges Hérelle.

Percorso non lineare, ricco di sorprese e intersezioni, quello che porta a ricostruire i rapporti intercorsi tra Gabriele d'Annunzio e Matilde Serao, scrittori e giornalisti, personalità curiose di un tempo a cavallo fra due secoli le cui vite, più volte, si intersecarono sia per motivi strettamente lavorativi sia per ragioni private, dovute alle relazioni del Vate con alcune donne amiche di Serao.

La critica, sinora, ha più volte evidenziato punti di contatto e motivi speculari che sembrano proporsi a conferma di un sodalizio amicale e fraterno, proseguito per circa quarant'anni, non privo di alti e bassi. Quest'articolo intende proseguire in quella direzione e ricostruire alcune tappe del rapporto fra i due grazie al Fascicolo Serao conservato alla Fondazione Il Vittoriale degli Italiani e a saggi pregressi che affrontano il tema. I materiali contenuti nella suddetta cartella, anche se in numero esiguo, portano all'attenzione per lo più un dialo-

$\begin{array}{lll} & \text { Peer review } & \\ & \text { Submitted } & 2019-02-23 \\ \text { Edizioni } & \text { Accepted } & 2019-07-15 \\ \text { Ca'Foscari } & \text { Published } & 2019-10-15 \\ & \text { Open access } & \end{array}$

@ 2019 | @(C) Creative Commons Attribution 4.0 International Public License 
go quotidiano e personale, non privo di elementi d'interesse, che intrecciano biografia e opera.

È probabile che Serao e d'Annunzio abbiano scambiato riflessioni di diversa natura soprattutto a voce o ne abbiano lasciato testimonianza in altri luoghi, ancora da scoprire. Soprattutto, considerando la lunga conoscenza, si presumono contatti frequenti con mezzi diversi dalle lettere - ad esempio il telefono. Non mancano, nella loro corrispondenza, valutazioni sugli scritti del poeta, informazioni di carattere pratico e una familiarità che rende vivo quel solido rapporto d'amicizia, costruito senza dubbio nel tempo: un rapporto che cela luci e ombre.

Come si noterà, d'Annunzio fu un punto di riferimento anche per la famiglia dell'autrice napoletana; i suoi figli lo consideravano una "figura di casa" quasi paterna, tanto che, alla morte della madre nel luglio 1927, inviarono un telegramma accorato a Gardone Riviera avvertendolo dell'accaduto.

Come anticipato, nel tracciato che si propone si considereranno pubblicazioni pregresse che hanno messo al centro la presenza, ad esempio, di Georges Hérelle, traduttore del Vate e in contatto con Serao e il marito e scrittore Edoardo Scarfoglio, come indicato da Raffaele Giglio in uno studio del 1977. Altra figura di rilievo pare invece essere stato il conte Robert de Montesquiou, con cui i due intrattennero uno scambio privato negli stessi anni: la comune conoscenza nella $\mathrm{Pa}$ rigi dei primi Novecento è stata proposta, con una pubblicazione dei carteggi, da parte di Pierre De Montera e Guy Tosi ancora negli anni Settanta cui si farà accenno. Volumi più recenti, riguardanti la lettura dell'opera di Serao e del suo contesto di riferimento, hanno ristabilito come necessaria la possibilità di parlare di d'Annunzio attraverso la lente della vita della scrittrice. Inoltre, la prospettiva autobiografica di uno e dell'altra fornisce indicazioni circa l'interpretazione che entrambi danno al proprio lavoro.

Il Fascicolo Serao riporta tre lettere e una busta; sei telegrammi; una cartolina e una ricevuta. Se nel testo dell'articolo sarà presentata una trascrizione, in appendice si propone una scansione dei documenti autorizzata dal Vittoriale. Essi forniscono altri dettagli concernenti il tempo e le relazioni dell'autore con diversi artisti e studiosi, o con altri soggetti, tra cui Eleonora Duse e la Marchesa Casati. Non si rintracciano, tra questi materiali riguardanti il lavoro del Vate, ad esempio, quelli per il quotidiano Il Mattino fondato da Scarfoglio e Serao.

Per ragioni che riguardano probabilmente la fiducia nei confronti di Matilde, nella stessa cartella dell'Archivio si conservano alcune lettere inviate da lei a Luigi Lodi, letterato vicino a Carducci che, grazie a quest'ultimo, ottenne dal 1883 di far parte della redazione del giornale Capitan Fracassa, dov'erano inseriti già sia d'Annunzio sia Scarfoglio. Le missive private testimoniano la relazione amorosa tra Serao e Lodi, forse assecondata in segreto dal Vate, il quale frequentò da vicino anche la moglie del collega, la giornalista Olga Os- 
sani, meglio conosciuta nei carteggi dannunziani con lo pseudonimo di Febea e probabile figura ispiratrice del personaggio di Elena Muti nel Piacere. Anche Serao ebbe modo di conoscerla e di scriverle: una lettera è indirizzata anche a lei. È stata Mariadomenica Verde (2004) a analizzare con puntualità gli estremi delle missive di Serao a Lodi, testimoniando quella relazione accennata nella sua monografia da Anna Banti (1965). La studiosa ha focalizzato da vicino il periodo romano di Serao, 1882-1884, che pare coincidere con il contenuto delle lettere e, soprattutto, con la frequentazione del Capitan Fracassa e con il completamento de La conquista di Roma (1885), romanzo che ebbe un discreto successo. Le ragioni della conservazione delle lettere a Lodi nel fascicolo del Vittoriale possono essere soltanto ipotizzate, dal momento che non hanno nulla a che fare con il proprietario, il quale potrebbe aver mantenuto il riserbo dell'amica salvaguardandola da dicerie. ${ }^{1}$ Ce n'è una, in particolare, in cui Serao invita Lodi a restituirle le lettere scritte; lei avrebbe dovuto fare altrettanto. Eppure ciò non accadde, così come nel Fascicolo non si conservano risposte da parte di lui.

Un altro documento risalente al 1921 risulta forse estraneo a questo gruppo: una lettera rivolta a d'Annunzio da Bice di Colloredo Mels del Torso, figlia del conte e nobile Antonino, signore di Colloredo, Mels e ville annesse. L'inclusione delle precedenti si può ipotizzare essere stata una scelta di d'Annunzio, alla luce di contenuti affini che si andranno via via affrontando nelle prossime pagine. Di certo il milieu comune del Vate e di Serao permette di riconsiderare alcuni passaggi salienti e esporre riflessioni significative nonché prospettive di lettura aggiornate, talvolta chiarificatrici.

I due si conobbero probabilmente durante l'esperienza romana del già citato Capitan Fracassa, su cui il Vate pubblicò la cronaca delle nozze di Matilde con Scarfoglio nel 1885. Quando i coniugi si stabilirono a Napoli, dal 1888, iniziarono a collaborare al Corriere di Napoli e fondarono poi Il Mattino nel 1892. A entrambi partecipò anche d'Annunzio che, dal 1891, iniziò a soggiornare in città rimanendovi fino al 1893. Saranno composti lì anche i romanzi l'Innocente (che uscirà prima per Bideri di Napoli nel 1892, quindi per Treves) e Il trionfo della morte (la prima edizione sarà per Treves nel 1894). Il primo sarà tradotto da Hérelle in francese nel 1892 sulla rivista Le Temps (in volume nel 1893) mentre Il piacere (L'enfant de volupté) era già stato affrontato qualche tempo prima dallo stesso traduttore.

Sono quegli gli anni in cui si concentrano gli incontri con personaggi illustri; oltre a Hérelle anche Henry James, che manterrà un ricordo di

1 Ringrazio gli archivisti del Vittoriale Alessandro Tonacci e Roberta Valbusa per l'aiuto fornitomi. Una verifica da loro svolta porta a confermare che non si conoscono i modi attraverso cui sia stato composto il Fascicolo. 
Serao e d'Annunzio, come conferma Giorcelli $(1968,103)$. La studiosa richiama gli anni in cui il Conte Primoli aveva introdotto i due letterati all'autore americano; il loro incontro, come testimoniato in una lettera del 18 giugno 1894, ebbe luogo - dato degno di nota - a Venezia, dove d'Annunzio, nell'agosto, incontrò anche Hérelle per la prima volta.

Il tentativo di questo articolo sarà di definire ulteriori contorni attorno alle lettere di Serao che saranno proposte, cercando le corrispondenze con quanto la critica ha già affrontato. È senza dubbio complesso renderle parlanti senza possedere le risposte di d'Annunzio, non rintracciabili nel Fascicolo citato. Non esistendo né un Archivio Serao né un Fondo dedicato alla scrittrice non è stato possibile accertarsi dell'esistenza odierna delle risposte del poeta. Eppure si può partire dalla cronologia che antecede le missive, ossia dalla dedica rivolta all'amica esposta come prefazione al Giovanni Episcopo pubblicato nel 1892 proprio a Napoli da Pierro. ${ }^{2}$ Non è un caso che il breve romanzo di d'Annunzio arrivi, in quegli anni napoletani, con dedica alla scrittrice; lei, precocemente, aveva iniziato a pubblicare su Il Mattino alcuni feuilleton tradotti da Honoré de Balzac e Guy de Maupassant, e brani da Dostoevskij, facendo conoscere ai propri lettori quell'orizzonte letterario internazionale di cui d'Annunzio faceva parte. La trama, ispirata alla forma del racconto-confessionale, tra dramma familiare e omicidio sul modello degli autori russi che si stavano diffondendo in Europa, poteva di certo essere compresa e apprezzata da Serao, donna aggiornata sulle tendenze letterarie in auge. Si legga dunque la premessa:

\section{A Matilde Serao.}

Illustre signora, mia cara amica, questo piccolo libro che io vi dedico non ha per me importanza di arte; ma è un semplice documento letterario publicato a indicare il primo sforzo istintivo di un artefice inquieto verso una finale rinnovazione.

Fu scritto a Roma nel gennaio del 1891, dopo quindici mesi di completo riposo intellettuale trascorsi in gran parte fra ozii torpidi e esercizii violenti dentro una caserma di cavalleria. La persona di Giovanni Episcopo era già stata da me osservata e studiata con intensa curiosità, due anni innanzi. Il filosofo Angelo Conti l'aveva conosciuta per la prima volta nel gabinetto d'un medico, all'ospedale di San Giacomo. Io, quel nobile filosofo e il pittore simbolico Marius de Maria avevamo poi frequentato una mortuaria taverna della via Alessandrina per incontrarci col doloroso bevitore. Alcune cir-

2 Una copia è conservata presso la Biblioteca della Fondazione Giorgio Cini di Venezia. È da precisare che, secondo una ricerca nell'OPAC nazionale, la stessa opera uscì forse nello stesso 1892 a Lanciano per Carabba. 
costanze bizzarre avevano favorito il nostro studio. (Angelo Conti appunto aveva provveduto la siringa e la morfina pel povero Battista!) Ma il raro materiale, raccolto con la maggior possibile esattezza, era rimasto grezzo in alcune pagine di note.

Voi, così costante e così fiera lavoratrice, non conoscete forse i gravi turbamenti che porta nella conscienza dell'artefice una lunga interruzione del lavoro. Uscito dalla servitù militare, io durai fatica a riprendere le antiche consuetudini dello spirito, a acquistare una nozione precisa del mio nuovo stato interiore, a raccogliermi, quasi direi a ripossedermi. Compresi allora come sia profonda e inevitabile su noi l'azione pur degli estranei da cui tante diversità ci separano, e come sia più difficile preservare la nostra persona morale che il nostro corpo dai rudi contatti delle moltitudini per mezzo a cui viviamo o passiamo. Nulla, mia cara amica, nulla di quanto crediamo nostro ci appartiene.

Nell'incipit del testo d'Annunzio riporta notizie circa il tempo della scrittura, il modello realistico cui il personaggio principale si rifà - nodo critico che sarà centrale di qui in avanti - e un periodo di mancata ispirazione che precedeva l'arrangiamento dell'opera in questione. Curiosa è la presenza del filosofo Angelo Conti, che d'Annunzio aveva incontrato al Capitan Fracassa e figura esperta d'arte, con cui si avviò un dialogo per lui proficuo nel tempo rispetto alle tendenze introdotte in Italia e che avrebbero poi fatto parte del simbolismo pittorico italiano. Nella prosecuzione estende i motivi del vuoto lavorativo e spiega in che modo sia giunto a una svolta letteraria:

Mi pareva che tutte le mie facoltà di scrittore si fossero oscurate, indebolite, disperse. Mi sentivo in certe ore così profondamente distaccato dall'Arte, così estraneo al mondo ideale in cui un tempo avevo vissuto, così arido, che nessuna instigazione valeva a scuotermi dall'inerzia pesante e triste in cui mi distendevo.

Qualunque tentativo riescì vano: nessuna lettura valse a fecondarmi. Le pagine predilette, che un tempo avevano provocato nel mio cervello le più alte ebrezze, ora mi lasciavano freddo. Di tutta la mia opera passata provavo quasi disgusto, come d'una compagine senza vitalità, la quale non avesse più alcun legame col mio spirito e pure mi premesse d'un intollerabile peso. Certi brani di stile, in qualche mio libro di prosa, mi facevano ira e vergogna. Mi parevano vacue e false le più lucide forme verbali in cui m'ero compiaciuto. Mai artefice ripudiò la sua opera passata con maggior sincerità di disdegno, pur non avendo ancóra in sé l'agitazione dell'opera futura né la conscienza del nuovo potere.

$\mathrm{Ma}$ in noi esseri d'intelletto un lavorio occulto si compie, le cui fasi lente non sono percettibili talvolta neppure in parte dai più vi- 
gili e dai più perspicaci. Se sul nostro intelletto pende di continuo la minaccia spaventevole o d'una improvvisa lesione o d'una progressiva degenerazione degli organi, in compenso questi medesimi fragili mutevoli organi sono mossi al servizio dell'Arte da attività misteriose e prodigiose che a poco a poco elaborano la materia quasi amorfa ricevuta dall'esterno e la riducono a una forma e a una vita superiori. E l'una e l'altra possibilità, la tragica e la felice, hanno comune il campo oscuro ed immensurabile della nostra inconscienza bruta.

Una sera di gennaio, stando solo in una grande stanza un poco lugubre, io sfogliavo alcune raccolte di note: materiale narrativo in parte già adoperato e in parte ancóra vergine. Una singolare inquietudine mi teneva. Se bene io fossi occupato alla lettura, la mia sensibilità era straordinariamente vigilante nel silenzio; e io potei osservare, nel corso della lettura, che il mio cervello aveva una facilità insolita alla formazione e alla associazione delle imagini più diverse. Non era quella la prima volta che accadeva in me il fenomeno, ma mi pareva che mai avesse raggiunto un tal grado d'intensità. Incominciavo a vedere, in sensazione visiva reale, le apparenze imaginate. E l'inquietudine si faceva, di minuto in minuto, più forte.

La lunga confessione che anticipa la forma del romanzo espone il decadentismo di d'Annunzio autore ma anche le derive della nascita di questo suo lavoro. La mancanza di relazione con l'Arte, prima, e il guizzo di risoluzione successivo quasi dichiarano - con una sorta di captatio benevolentiae rivolta al lettore, che avrebbe poi tenuto da conto questa dedica a stampa - da un lato l'evoluzione personale e lavorativa dell'autore, interna a un 'fare' - almeno in quel momento - pregno d'incostanza, dall'altro l'intuizione che sta alla base della costruzione del libro. L'unione di materiale edito e inedito ma anche la formula dell'inquietudine' come tensione necessaria per metter mano alle «note» sono gli strumenti di cui egli si serve per l'elaborazione. La creazione del personaggio, dalla realtà alla pagina scritta, con tanto di nomi propri e riferimenti a eventi precisi, d'Annunzio la spiega in questi termini:

Quando lessi sul frontespizio di un fascicolo il nome di Giovanni Episcopo, in un attimo, come nel bagliore d'un lampo, vidi la figura dell'uomo: non la figura corporea soltanto ma quella morale, prima di aver sotto gli occhi le note, per non so qual comprensiva intuizione che non mi parve promossa soltanto dal risveglio repentino d'uno strato della memoria ma dal segreto concorso di elementi psichici non riconoscibili a alcun lume d'analisi immediata. Allora quell'uomo dolce e miserabile, quel Christus patiens, si mise a vivere (innanzi a me? dentro di me?) d'una vita così profonda che la mia vita stessa ne restò quasi assorbita.

Mai, signora, mai da creatura terrestre avevo ricevuta una più violenta commozione. Mai avevo assistito a un più alto e più spon- 
taneo miracolo dell'intelligenza: alla perfetta ricostituzione d'un essere vitale nello spirito di un artefice repentinamente invaso dalla forza creatrice. Mai Giovanni Episcopo era stato più vivo.

E con lui Giulio Wanzer, Ginevra, Ciro, il vecchio, respiravano, palpitavano: avevano i loro sguardi, i loro gesti, le loro voci, un odore umano, qualche cosa di miserevolmente umano che doveva rendere indimenticabili i loro aspetti. E ciascun episodio del dramma doveva aver la potenza di suscitare un brivido non somigliante a alcun altro. E quella corsa del padre e del figlio, sotto il sole feroce, nel silenzio, nel deserto, a traverso i terreni ingombri di macerie, fra le pozze di calce abbacinanti; e quel loro entrare nella casa muta, luminosa e vacua; e quell'aspettazione misurata mortalmente dai palpiti delle loro arterie; e il grido selvaggio, e il fanciullo avviticchiato al gran corpo di quel bruto, e i colpi di coltello in quella schiena possente, e lo schianto, e il gorgoglio del sangue; e l'agonia di Ciro, in quella stanza, nel crepuscolo, al conspetto dell'ucciso; e poi, nell'ore che seguirono, il padre solo con quei due cadaveri...

A ben vedere la costruzione narrativa della premessa, estetizzante e frenetica, si allinea al testo, nel segno di un impulso creativo composto dall' intuizione' e dalla 'memoria di eventi psichici non riconoscibili' ma anche di quell'«innovazione» che già Serao desiderava per il suo giornale, e che sarà continuata da d'Annunzio con le opere successive. La rivelazione di un argomento trascurato e degno di essere ripreso, dalle note al romanzo, è ciò che mette in moto il lavoro:

Ah, mia cara amica, perché ebbi una sì fiera visione e feci una sì debole opera? Perché su la pagina quel gran tutto di forza si attenuò e si spense?

La mattina dopo, mi misi al lavoro. Lavorai con una strana energia, per alcuni giorni, senza altra interruzione che quella del sonno e dei pasti. E avevo sempre d'innanzi agli occhi viva, specialmente nella notte, la figura di Giovanni.

Ecco, mia cara amica, la genesi di questo piccolo libro che io vi dedico. Penso che troverete qui i primi elementi di una rinnovazione proseguita poi nell'Innocente con più rigore di metodo, esattezza di analisi, semplicità di stile.

Tutto il metodo sta in questa formula schietta: - Bisogna studiare gli uomini e le cose DIRETTAMENTE, senza transposizione alcuna.

Ma chi vorrà studiare? Quanti ancóra in Italia intendono il significato di un tal verbo? Quanti sentono la necessità di rinnovarsi? Quanti hanno fede nella loro forza e sicurezza nella loro sincerità?

Pure, non mai come oggi fu imperioso il dilemma: - O rinnovarsi o morire.

A voi, signora, a voi che ricercando il meglio date in Italia l'esempio di una operosità così virile, dedico dunque un documento publi- 
cato a indicare il primo sforzo istintivo di un artefice inquieto; il quale tanto è appassionato dell'Arte che non può rassegnarsi a morire.

G. d'A.

Napoli: nell’Epifania del 1892.

In studi recenti a cura di Verdile (2017) e Rocco Carbone (2018) si conferma che Serao non percepiva affatto la propria operosità come «virile». La sua postura ferma era quella di donna conscia dei propri mezzi, tanto sicura di sé da esporre la propria soggettività senza timore. D'Annunzio la definì anche una «costante e fiera» lavoratrice.

Gli strumenti della giornalista e della scrittrice, nel 1892, erano già collaudati e lei poteva vantare numerose pubblicazioni a suo nome, nonché numerosi riconoscimenti. Ciò che interessa, in questo caso, è la direzione del discorso che d'Annunzio dà, portando all'attenzione la doppia presenza in antitesi di 'innovazione-morte' che vige anche nella sua poetica, provocando gli studiosi del tempo e aggiungendo una domanda che può riferirsi anche a Serao: «Quanti hanno fede nella loro forza e sicurezza nella loro sincerità?». Inoltre, attraverso la 'formula schietta' "Bisogna studiare gli uomini e le cose DIRETTAMEN$T E$, senza transposizione alcuna», rimarca il valore assoluto di un'indagine in presa diretta, parallela al positivismo, che aveva dato vita al Verismo in quegli anni e cui d'Annunzio pare rifarsi in termini di realismo, ma che è ancora lontana da quel simbolismo che sembrerà abbracciare in seguito e che culminerà con Il trionfo della morte (1894).

Secondo una nota formula, infatti: «tra il 1892 e il '93 gli scritti critici di d'Annunzio sono tutti da leggere in rapporto a questa trasformazione di gusto e di ideologia che segna insieme la fine del naturalismo» (Raimondi 1976, 36); lo scarto individuato qui pare non accordarsi con la dedica-premessa al Giovanni Episcopo o, addirittura, segnalare per mano dello scrittore la fase di rimaneggiamento dei propri testi. Nell'Innocente egli indica un miglioramento dovuto a «più rigore di metodo, esattezza di analisi, semplicità di stile» di quello applicato al Giovanni Episcopo, che sarà ripreso per essere pubblicato a breve distanza dal sopraccitato romanzo. Tuttavia la dichiarazione di poetica pare sottratta al simbolismo: risulta, anzi, 'fuori campo' e, per questa ragione, d'interesse. A proposito della prima opera, si veda l'analisi di Giammarco (2004).

Circa le corrispondenze fra il romanzo di d'Annunzio e la trasposizione filmica Il delitto di Giovanni Episcopo del 1947 di Alberto Lattuada ci si può rifare all'esaustiva lettura di Borin (2017), il quale guarda all'una e all'altra opera con un confronto aperto fra linguaggi, personaggi e temi, proponendo un repertorio di similitudini e differenze. Dall'originale dannunziano è tratto anche il dramma Episcopus di Puppa (2005), creato per una trasposizione teatrale del testo. 
Per tornare invece al Fascicolo: la prima lettera di Serao coincide con una parte del carteggio con Hérelle, ma può essere anche letta separatamente. Il volume di Giglio, tuttavia, fa da cartina di tornasole a certi passaggi di lavoro. Da esso si ricavano alcuni dettagli: d'Annunzio e l'intellettuale francese si erano già incontrati nel 1894; invece Serao e Scarfoglio l'avevano conosciuto una prima volta a Napoli già nel 1891; lei intratterrà poi con Hérelle rapporti epistolari dal 1895 ai primi anni del Novecento. Ė indispensabile leggere alcuni argomenti trattati intersecando i diversi carteggi, per riconoscere quale fecondo rapporto di stima vi fosse tra i tre, come si vedrà.

Napoli, lì 18 dicembre 1898

Carissimo Gabriele,

mi sono immediatamente occupata del vostro passaggio sul Margherita per Alessandria d'Egitto: oggi è domenica quindi non ho potuto avere nessuna risposta ma da domani, lunedì o dopodomani, io posso telegrafarvi il tutto a Settignano: avrete poi il tempo di arrivare comodamente a Napoli per sabato. Il vecchio Treves caverà fuori le sue monete che, certo, egli fonde come gli antichi ebrei suoi antenati? Voi siete degnamente il suo enfant cherì: e non dubito dell'esito.

Quanta gioia procurerà alla nostra amica il vostro arrivo, colà! Mio carissimo Gabriele, ella non solo vi ama quanto è possibile amarci, in tutte le forme della tenerezza e della passione muliebre, ma la ritengo la sola donna capace di amarvi bene, come si deve amare voi: la sola donna capace di comprendere, rispettare, adorare il vostro genio e il vostro amore. Le sue belle mani non possono che comporre il vostro bene e non vi domandano, in cambio, che quella dolcezza consolatrice di cui ella ha bisogno!

La Gioconda è un'opera di vita. La celeste figura di Silvia vinta strappa le lacrime: ma anche Lucio e Gioconda hanno ragione. L'hanno: tutti hanno ragione, nella vostra tragedia perché la esistenza è così, perché il poeta deve sentire tutte le voci umane. Testualmente parlando - il tutto è una schifosa cosa, ma dobbiamo considerarlo - ritengo il secondo e terzo atto magnifici. Forse è più bello, poeticamente parlando, il quarto: a me, solingamente, piace più di tutti. L'amica nostra renderà questo quarto atto in maniera divina. Beata astuzia vostra, con cui avete scritto questa cara Gioconda!

Edoardo è a caccia nel San Gaal, cioè nella foresta di Monticchio: tornerà martedì.

Vogliatemi bene

Matilde $^{3}$

3 In Fondazione Il Vittoriale degli Italiani, Archivio Iconografico, Serao, Matilde - XLII 3. Lettera manoscritta datata come da indicazione; presenta le sottolineature riportate. 
È quello il periodo in cui l'autore si trova a Settignano, nella sua Villa La Capponcina (già dai primi mesi del 1898) ma è anche il momento che precede il passaggio da Napoli per imbarcarsi e raggiungere Alessandria d'Egitto il 24 dicembre 1898, dove, come si sa, lo attendeva Eleonora Duse. Serao si occupa di organizzare i viaggi e vigilare sui fatti privati del poeta, con fare di complice. In questa lettera, inoltre, Serao dapprima invita d'Annunzio a chiedere del denaro a Treves - loro comune editore - e, in seguito, fa di certo riferimento alla travagliata relazione con l'attrice, che mise in scena appunto, nel 1899, La Gioconda. Di lei amica, oltre che tra le interpreti predilette del Vate, sembra incarnare l'amante congeniale dal punto di vista di Serao, che ne illustra le qualità con un certo orgoglio. Particolare risulta anche l'approccio alla lettura privata del testo della tragedia citata: la scrittrice è dunque un'interlocutrice privilegiata del poeta e, come altri, legge in anteprima le sue opere.

Il carteggio tra la Serao e la Duse è stato pubblicato da Tortora (2004); in esso si rintracciano, tuttavia, solo materiali più tardi: un telegramma del 1901 e alcune missive inviate alla Duse tra il 1920 e il 1921; mancano invece quelle a Serao. La studiosa ha ipotizzato degli scambi più frequenti nello stesso periodo indicato e possono essere plausibili anche materiali che vadano dal 1878 - anno della loro mutua conoscenza a Napoli - al 1920. Negli anni Venti la relazione con d'Annunzio non è materia di conversazione; lo sono invece il lavoro d'attrice di Eleonora, l'esperienza di vita di entrambe e l'elogio di Serao nei confronti del lavoro dell'amica.

L'intenso epistolario coevo con Hérelle, come già asserito e come riporta Giglio, non contiene lettere di d'Annunzio, raccolte invece da Tosi (1946). La ricostruzione del primo studioso permette di conoscere soprattutto quali fossero i giudizi sull'opera del poeta abruzzese ma anche circa le sue relazioni sociali.

Un passaggio chiave risulta quello della lettera del 15 maggio 1899, in cui Serao nomina La Gioconda come opera riuscita, mentre della tragedia La Gloria «non ne parliamo, abbiamo sofferto enormemente per quella» (Giglio 1977, 160). Nella stessa lettera si indica il bisogno di denaro di d'Annunzio, che aveva iniziato a occuparsi di libretti d'opera. Entrambe rappresentate dalla Duse, le opere paiono strettamente legate alla relazione con lei anche nelle parole di Serao, la quale più tardi, il 6 gennaio 1900, scriverà ancora a Hérelle:

Nessuna notizia di Gabriele. Dopo l'insuccesso della Gloria, a Napoli, ha finito per odiare questo paese: allo stesso modo, credo, ami poco anche noi, che abbiamo fatto il possibile per salvarlo! Credo 
che la mia grande Amica sia nuovamente alla Capponcina: questa relazione li rende molto infelici, tutti e due. Non posso pensarvi, che con grande tristezza. (167)

Come si ricostruirà dalla lettura dell'intero epistolario, tra Serao e la Duse non vi saranno sempre rapporti distesi, forse a causa di quest'ultimo appunto. Il 14 gennaio, tuttavia, ancora lei scriveva: «Non potete credere che piacere io provi per lui, vedendolo finire il Fuoco; uscirà da questa terribile empasse, vittorioso, farà degli altri romanzi, evviva, evviva!» (168). L’appoggio all'amico, dunque, resiste.

In quel momento Hérelle traduce in francese anche Grazia Deledda; un altro dei suoi autori sarà Fogazzaro. La restante corrispondenza, infatti, oltre a alcune opere di Serao e altre attività che li vedono coinvolti, non menziona d'Annunzio fino al 1906.

Son da riportare, dunque, le successive lettere di Serao al Vate dal Fascicolo di cui ci si sta occupando, che risalgono invece al biennio 1903-1904. Questi documenti indicano l'attenzione riservata dall'autrice al lavoro dell'amico, in linea con quelle al traduttore:

28 avril 1903

\section{Caro Gabriele,} viene da voi, a Settignano, un gentiluomo francese, della grande società parigina, Georges Rodier: giovine intelligentissimo, innamorato dell'Italia, della poesia italiana e quindi innamoratissimo di voi! Potete aprirgli la casa e tendergli la mano: non vuole né pubblicare una intervista né descrivere i vostri mobili!

È vero che l'Amica è malata a Rapallo? Le scrivo!

E le Laudi?

Vogliatemi bene

Matilde Serao ${ }^{4}$

Serao in poche righe propone almeno due punti chiave. Il primo: l'incontro con il filosofo Georges Rodier, che sarà docente universitario presso la Sorbona di Parigi. ${ }^{5}$ La datazione manoscritta fa pensare a un soggiorno francese di Serao in quel momento, la quale spedisce all'amico una breve missiva per annunciargli l'arrivo di Rodier. Ancora

4 Ivi, con medesima segnatura come i prossimi documenti citati.

5 La lettera indica chiaramente questo nome. Il filosofo era nato nel 1864 e apparteneva alla generazione precedente rispetto a quella di Serao; tali dati si possono desumere dal catalogo della Bibliotéque Nationale de France: https://data.bnf.fr/fr/12400012/ georges_rodier/ (2019-02-21). Nella stessa cartella si rintraccia una missiva firmata dallo stesso - si presume - George Rodier che non riporta l'anno ma la data del «23 janv.»; qui Rodier ringrazia per le dediche personali e dice di sentirsi soddisfatto rispetto all'incontro. 
una volta la scrittrice indica il titolo di un'opera letta in anteprima: le Laudi, che usciranno per Treves di lì a pochi mesi, e che proprio nella primavera del 1903 trovano un loro compimento.

Infine: la formula di congedo «Vogliatemi bene» è ripresa anche a distanza di tempo, segno di un rapporto stabile e duraturo. In effetti, la presenza dell'«Amica» fa intendere quella complicità già postulata, ovvero la possibilità che Serao facesse da anello di congiunzione fra il Vate e la Duse, con cui intratteneva ancora una relazione in quegli anni, come testimonia Guerri (2008), pur avendo già incontrato Luisa Casati. Si ipotizza dunque, in questa sede, che l' «Amica» sia ancora Eleonora Duse.

Meno di un anno dopo, Serao torna a scrivere a d'Annunzio:

Carissimo Gabriele, esco[no] due dispacci - ieri e oggi - dell'Amica. Hélas, essa è veramente malata! Andrò a vederla fra quattro o cinque giorni, di ritorno da Milano.

Restituitemi i due dispacci ma mettete un motto e la firma sopra questo foglio: esso serve per la Duchessa di Fiano, la fulgida beltà! Vi benedico teneramente,

Matilde Serao

L'autorevolezza di Serao emerge nella fermezza delle richieste ma è anche d'interesse il rimando alla Duchessa di Fiano, che d'Annunzio aveva incontrato durante il suo periodo di lavoro a Roma per La Tribuna e dunque negli anni Ottanta dell'Ottocento, come puntualizza Calderoni (2012); la studiosa si concentra sull'analisi degli articoli del Vate rivolti al mondo del costume da lui conosciuto nella Città Eterna.

Nel 1906, ancora nel carteggio di Serao a Hérelle, si fa riferimento alla Duse e a d'Annunzio. La prima poiché dedicataria e «anima gloriosa» di Dopo il perdono, la tragedia dell'autrice napoletana; a proposito di questa, che sarà tradotta in francese, l'affondo sul teatro dannunziano non manca ad arrivare in una lettera del 17 giugno 1906: «E d'Annunzio? Nessun romanzo? Nessuna pièce moderna? Ah, miseria delle miserie, la via degli artisti superbi e tristi, come lui!» (Giglio 1977, 206). Senza lusinghe Serao si riferisce al lavoro dell'amico, che pare giunto a un punto morto. Probabile che, in quel momento, lo fosse anche il rapporto tra i due, di cui né Banti né Giglio conoscono i risvolti successivi.

Dal contatto del 1898 - almeno secondo il Fascicolo del Vittoriale - trascorreranno diversi anni prima di rintracciare un nuovo segnale di avvicinamento; ad esempio, nel telegramma datato dall'Archivio 12-8-1912, inviato da Saint Moritz: «Matilde Serao e Luisa Casati non possono vivere senza di voi venite subito». Anche in questo caso, due donne centrali nella biografia del poeta si ritrovano insieme. Il 14 settembre 1912, con una cartolina da Interlaken, nel cantone di 
Berna, Serao scrive a d'Annunzio, allora alloggiato a Saint-Jean-deLuz, in Francia: «Vi mando, carissimo Gabriel, un fraterno saluto [...]. Fra quattro giorni sarò a Parigi, Hotel Lotti, e vi rimarrò due settimane. [...] Luisa Casati ed io e tutti vi abbiamo atteso vanamente a Saint Moritz. Amica vostra Matilde Serao». Il 27-9-1912 ancora Serao invia un altro telegramma manoscritto scrivendo: «Felicissima rivedervi rimango fino primo ottobre hotel Continental». In quegli anni, perciò, i due restano in contatto d'estate e si scambiano brevi messaggi per incontrarsi. Con loro anche la Marchesa Casati, che ebbe appunto in quel periodo una relazione con d'Annunzio.

È quello il tempo delle lettere a Robert de Montesquiou, che intrecciano anche quelle a Hérelle soprattutto dal lato di Serao, soprattutto nel 1904. Siamo all'interno del decennio di successo francese del Vate, da cui l'argomento delle principali missive al sopraccitato conte.

Lultimo gruppo di telegrammi dal Fascicolo Serao risale invece al 1926-1927. Il primo è manoscritto di lei al Vate e datato 22-11-1926: «Salute gioia e gloria al nostro grande fratello! Matilde Serao»; in quell'anno, il 21 giugno, vi fu la costituzione dell'Istituto Nazionale per l'edizione di tutte le opere di d'Annunzio. Questo messaggio pare dunque riferirsi a un evento più tardo: probabilmente alla monografia dedicata al suo lavoro da Francesco Flora, uscita in quell'anno presso l'editore napoletano Ricciardi, con cui l'autore antifascista suscitò la considerazione da parte del Vate, che lo definì «un nemico che vale la pena di avere».

Il 26 luglio 1927 d'Annunzio ricevette dai figli di Serao un telegramma manoscritto: «Madre nostra morta improvvisamente questa sera. Antonino Paolo Carlo Michele Scarfoglio»; a questo ne fa seguito un altro dell'11 agosto 1927: «In memoria della vostra grande amica ed ammiratrice vi abbracciano affettuosamente i suoi figli che sono anche i vostri figli. Antonino Paolo Carlo Michele Scarfoglio». Nel 1926 la scrittrice aveva dato alle stampe per Treves Mors tua, il suo ultimo romanzo, che la critica ha presentato più volte come un'opera d'ispirazione dannunziana, sebbene vi si rintracci quella che Filia ha descritto come un testo:

Liberato dalla retorica aulica della lingua letteraria italiana dell'epoca, da quel misto di realismo compassionevole e occhio decadente di un certo dannunzianesimo di maniera, questo romanzo sarebbe stato una lama ancor più affilata nel dramma della Grande Guerra. (2016)

C'è da credere che il lungo rapporto d'amicizia, costante e accorto da parte di lei, possa anche aver determinato un'influenza del poeta sul suo stile o forse un'adesione inconsapevole, come «ammiratrice».

Quanto fino a qui tracciato si propone come una focalizzazione di alcuni aspetti biografici e di lavoro che riguardano entrambi gli autori, 
in cui non mancano - trattandosi appunto di documenti privati - considerazioni personali, appunti di lavoro, richieste e aperture su questioni strettamente personali. Non sarà dunque improprio citare quella lettera del 1921 di Bice di Colloredo, in cui ella pareva proprio sostenere un'idea di femminile operoso, simile a quella incarnata da Serao: «Finiamola colle donne-corolles e mettiamo di moda la signorile dama-madre e donna, intelligentemente attiva!».

\section{Bibliografia}

Amedeo, Giovanni (2008). «Amori e fuga di Gabriele D’Annunzio durante il soggiorno napoletano». la Repubblica. URL https://ricerca.repubblica. it/repubblica/archivio/repubblica/2011/10/06/il-mondo-nascosto-che-annunzio-voleva.html (2019-02-20).

Banti, Anna (1965). Matilde Serao. Torino: UTET.

Calderoni, Elisabetta (2012). «D’Annunzio romano, dandy imperfetto». Poetiche, 14(36-3), 263-94.

D’Annunzio, Gabriele (1995). Giovanni Episcopo. A cura di Gianni Oliva. Roma: Newton Compton Editori. URL https://www.liberliber.it/online/autori/autori-d/gabriele-dannunzio/giovanni-episcopo/ (201902-20).

De Montera, Pierre; Tosi, Guy (1972). D’Annunzio, Montesquiou, Serao (documents inédits). Roma: Edizioni di storia della letteratura.

Filia, Francesco (2016). "Mors tua, Matilde Serao». Poetarum Silva, s.p. URL https://poetarumsilva.com/2016/11/21/mors-tua-matilde-serao/ (2019-02-20).

Giammarco, Marilena (2004). «Sul limitare dell'Ombra. Il giardino dannunziano

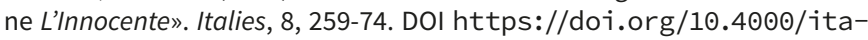
lies.1998.

Giglio, Raffaele (1977). Per la storia di un'amicizia: d’Annunzio, Hérelle, Scarfoglio, Serao. Napoli: Loffredo.

Giorcelli, Cristina (1968). Henry James e l'Italia. Roma: Ed. di storia e letteratura.

Guerri, Giordano Bruno (2008). D’Annunzio, l'amante guerriero. Milano: Mondadori.

Puppa, Paolo (2005). Episcopus. Molto liberamente tratto dal romanzo "Giovanni Episcopo" di Gabriele d'Annunzio. Salerno: Plectica Editrice.

Raimondi, Ezio (1976). «D’Annunzio e il simbolismo». Mariano, Emilio (a cura di), D'Annunzio e il simbolismo europeo = Atti del convegno di studio (Gardone Riviera, 14-15-16 settembre 1973). Milano: Il Saggiatore, 26-35.

Rocco Carbone, Lorenza (2018). Cara Matilde: la Serao, la scrittura e la vita. Napoli: Kairòs Edizioni.

Verde, Mariadomenica (2004). «Pretesto sopra un breve epistolario inedito di Matilde Serao a Luigi Lodi». Rupino, Angelo R. (a cura di), Matilde Serao: le opere e i giorni = Atti del convegno di Studi (Napoli, 1-4 dicembre 2004). Napoli: Liguori, 399-407.

Verdile, Nadia (2017). Matilde Serao. Lucca: Pacini Fazzi.

Tortora, Matilde (2004). Matilde Serao a Eleonora Duse: lettere. Napoli: Graus.

Tosi, Guy (1946). Gabriele D’Annunzio à Georges Hérelle: correspondance accompagnée de douze sonnets cisalpins. Paris: Denoël. 

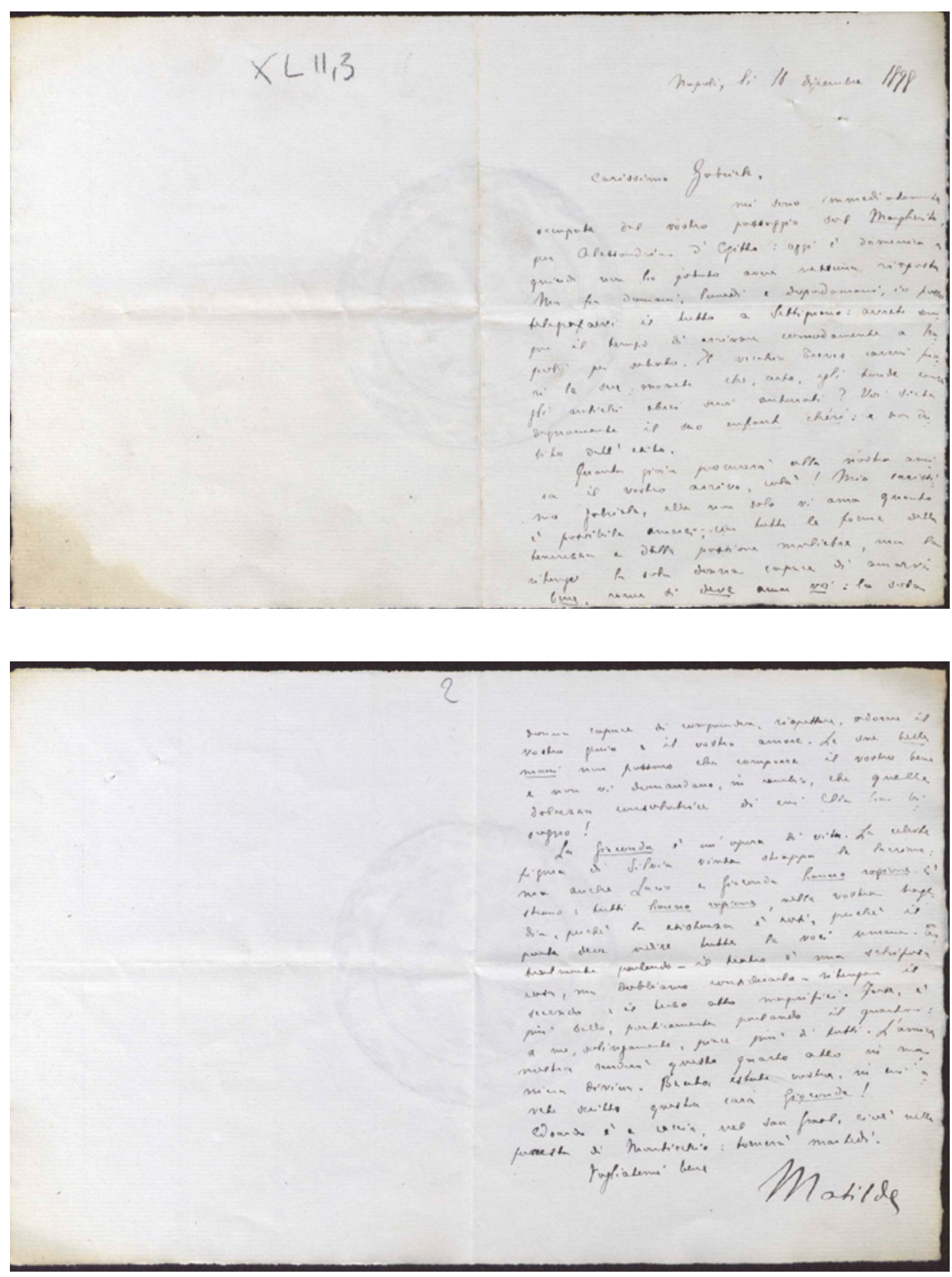
Alessandra Trevisan

«Vogliatemi bene»: lettere di Matilde Serao a Gabriele d'Annunzio

$g^{w^{3}}$

21 aval lyo3

Pare forisich,

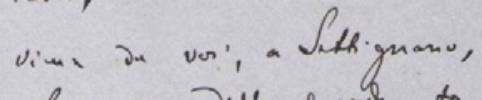

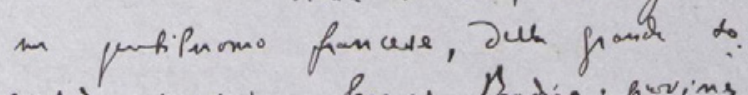
cith" pariginu, feonges Bodien: forvins

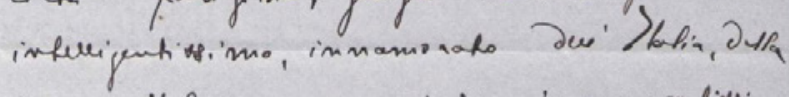

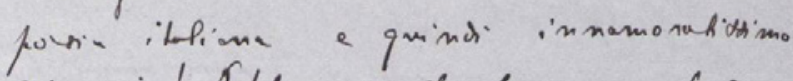

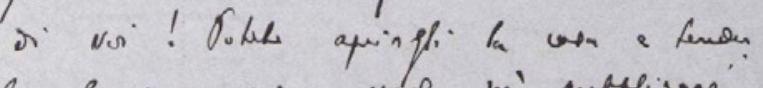

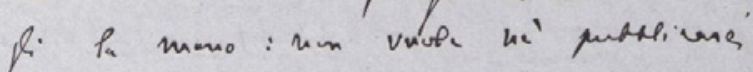

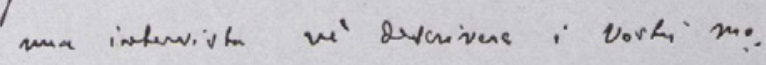

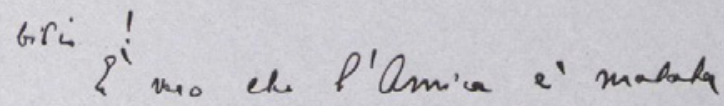
a Hopale? a verino! its tans?

Vopliatem. beve

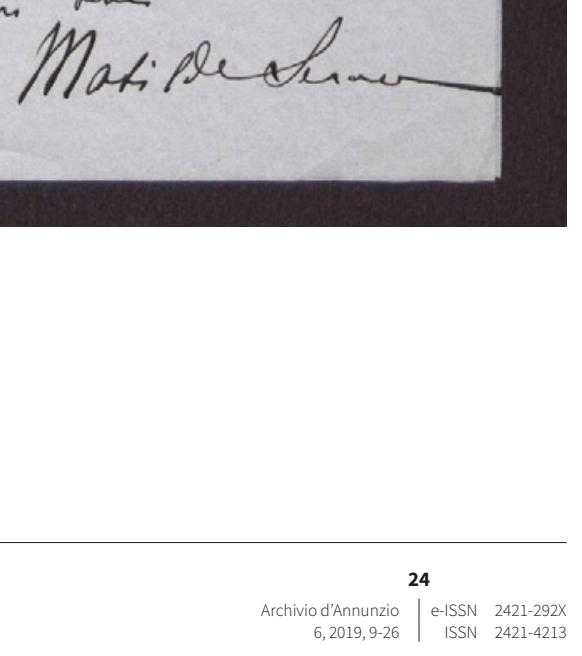




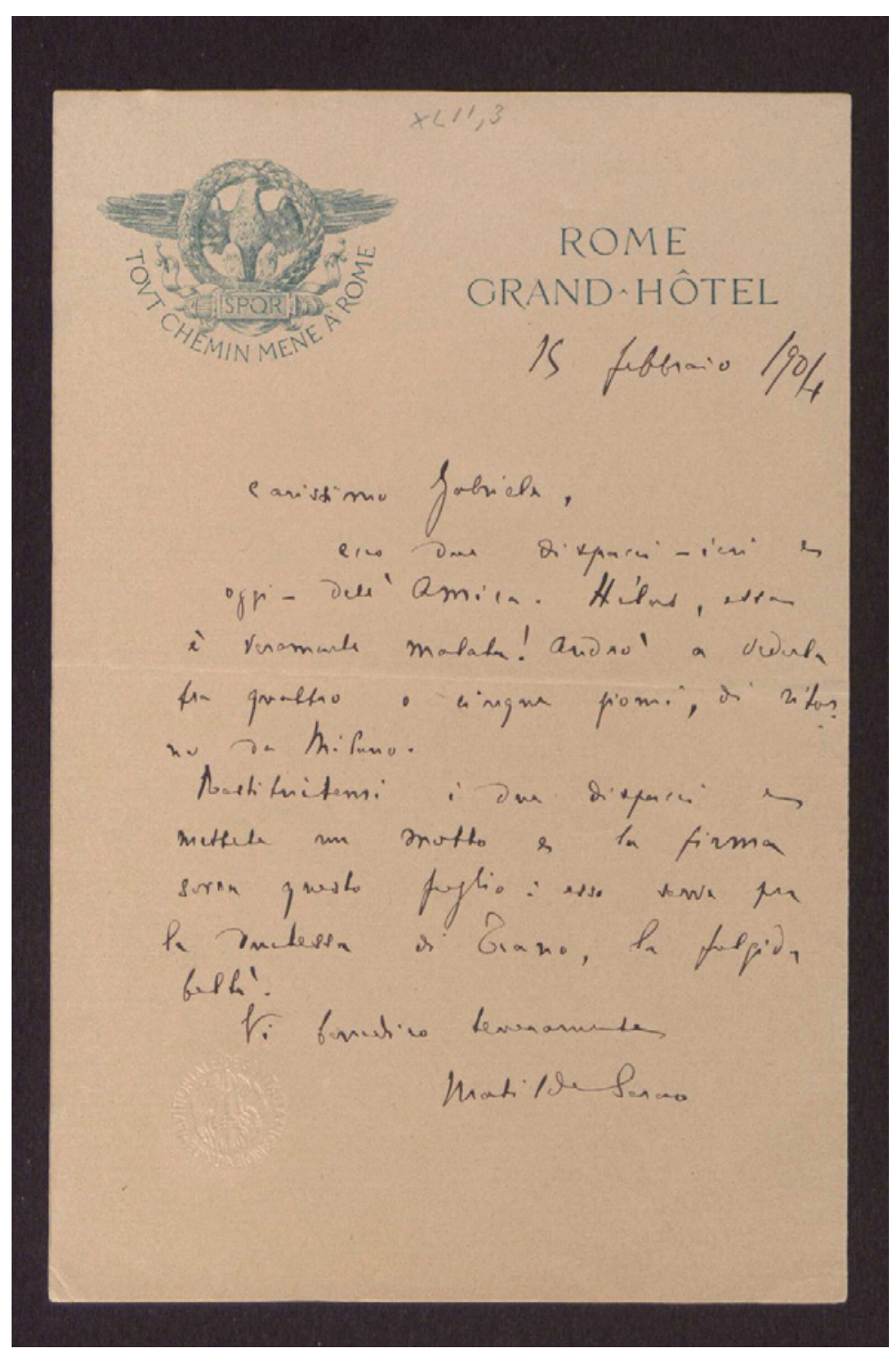





\section{Ferrara, Pisa, Ravenna: le «cittá soavi»}

Cristina Montagnani

Università degli Studi di Ferrara, Italia

Abstract Setting out from a poem that will be published as part of Elettra, this paper deals with the close relationship that exists among d'Annunzio's Laudi since their first appearance in 1899. As a case study, I will focus on Ferrara, Pisa, Ravenna, later published as Città del silenzio.

Keywords D’Annunzio. Maia. Elettra. Alcyone. Philology.

È l'autunno del 1899 quando d'Annunzio estrae dal crogiuolo i primi materiali delle future Laudi, ${ }^{1}$ e sono materiali di tutto rispetto: su $\mathrm{La}$ Nuova Antologia del 16 novembre 1899 (dieci giorni dopo la lettera a Giuseppe Treves ricordata in nota) viene pubblicato uno specimen di sette pezzi anepigrafi, preceduti dal titolo «Laudi del cielo del mare della terra e degli eroi» (Nuova Antologia, 84, 670: 195-212): I. Udite, udite, o figli de la Terra, udite il grande [L'Annunzio; secondo dei pezzi proemiali nella princeps di Maia, ma con funzione di prologo alle Laudi nel loro complesso] / II. [LII] Il mattino balzò, come la gioia di mille titani [Canto augurale per la nazione eletta] / III. [LXVIII] O deserta bellezza di Ferrara [Ferrara] / O Pisa, o Pisa, per la fluviale [Pisa] /

1 Il progetto laudistico si affaccia nell'estate dello stesso anno nelle lettere a Giuseppe Treves; qui d'Annunzio rievoca a uso dell'amico editore l'epifania delle prime laudi «figlie delle acque e dei raggi, tutte penetrate di aria e di salsedine» (lettera del 7 luglio. Oliva 1999, 547). Ma in novembre il poeta pensa già alle sue mitiche creature riunite in forma di libro: «Le Laudi si comporranno di sette libri: Alcione, Merope, Sterope, Celeno, Elettra, Taigete e Maia. I sette libri saranno divisi in tre volumi. Il primo - di prossima pubblicazione - si comporrà dei primi tre libri. Non è interamente compiuto, ma è composto in gran parte» (lettera del 6 novembre 1899, 554).

$\begin{array}{lll} & \text { Peer review } & \\ & \text { Submitted } & 2019-02-20 \\ \text { Edizioni } & \text { Accepted } & 2019-06-03 \\ \text { Ca'Foscari } & \text { Published } & 2019-10-15 \\ & \text { Open access } & \end{array}$

(C) 2019 | () Creative Commons Attribution 4.0 International Public License 
Ravenna, glauca notte rutilante d'oro [Ravenna] / IV. [LXX] Bocca di donna mai mi fu di tanta [Bocca d'Arno] / V. [LXXIX] Fresche le mie parole ne la sera [La sera fiesolana]. Si presentano tutti come pezzi lirici, finalizzati alla costruzione di una forma-canzoniere, e proprio per questo motivo lo specimen non accoglie frammenti di Maia, il poema che costituirà poi il primo libro delle Laudi. Ma sappiamo che d'Annunzio lavora all'inizio della Laus Vitae negli stessi giorni in cui prepara la silloge per la Nuova antologia: in occasione del matrimonio di Angiolo Orvieto con Laura Cantoni (18 ottobre 1899), nel «Quaderno di nozze» che gli amici del Marzocco offrirono agli sposi, ${ }^{2}$ leggiamo infatti, di mano del poeta, versi molto famosi (337-357): «Furonvi città soavi | su colli ermi, concluse | nel lor silenzio» eccetera, preceduti e seguiti da una significativa serie di puntini sospensivi. Versi che ci portano, alla stessa altezza cronologica dello specimen di canzoniere, nel cuore della prima parte della Laus.

Se tutto questo, dopo l'edizione critica di Maia (d'Annunzio 2006), è noto, può essere interessante studiare il serrato intreccio fra l'assieme di questi testi, o almeno una buona parte di essi, a partire, una volta tanto, da un componimento che confluirà in Elettra. Il rapporto fra Maia e Alcyone, infatti, è stato fatto oggetto di molti studi, paralleli alla elaborazione delle rispettive edizioni critiche (d'Annunzio 1988 e d'Annunzio 2006) o anche indipendenti da esse (penso soprattutto a Gavazzeni 1980), ma in questa complessa partita del dare e dell'avere Elettra ha finito con l'essere in sostanza esclusa, o quanto meno tenuta ai margini. La recente edizione a cura di Sara Campardo (d'Annunzio 2017), mi consente invece un piccolo focus che ha al suo centro Ferrara, Pisa, Ravenna, pubblicato del 1899 diviso nelle sue tre componenti, poi ricomposto, assieme ai più tardi sonetti sullo stesso tema, sotto l'etichetta complessiva delle Città del silenzio.

Il legame con i versi di Maia appena ricordati è evidente: le «città soavi», cui Maia riserva uno spazio piuttosto circoscritto, diventano in Elettra le Città del silenzio, destinate a ben altro sviluppo. Il rapporto con la Laus Vitae, però, è più profondo, sia perché coinvolge diverse porzioni testuali, sia perché condivide (spartisce?) con il poema parecchio materiale genetico desunto dai Taccuini, soprattutto da quelli ferraresi del novembre del 1898.

In maniera affatto analoga funziona il rapporto con Alcyone, che vede implicati testi compresi nello specimen laudistico della Nuova Antologia (soprattutto Bocca d'Arno), assieme al taccuino pisano del febbraio 1898.

2 Cf. Garoglio 1899, dove sono riportati i versi dannunziani; il Quaderno è oggi conservato presso il Fondo Orvieto dell'Archivio Contemporaneo A. Bonsanti del Gabinetto Vieusseux di Firenze (cassetta 229). Cf. Del Vivo, Assirelli 1983, 72; Del Vivo, Assirelli 1985. 
L'analisi comparata di questo materiale, e del regesto variantistico così come ce lo offrono le tre edizioni critiche, consente un affondo inedito, almeno quanto alla prospettiva, nell'officina del poeta in anni precocissimi rispetto alla elaborazione dei tre libri nel loro assetto finale.

Il primo dato che si impone con evidenza nel trittico di Elettra è il tema della lode, che a questa altezza cronologica è ben attestato anche in Alcyone, seppure in una forma più prossima al Cantico di San Francesco che alla lode stilnovistica. In ogni caso, le congruenze con La sera fiesolana, che è datata al 17 giugno 1899, sono profonde, al di là della ripresa «ti loderò»/«loderò» che fa pendant con l'incipit del ritornello della Sera: «Laudata sii». Accomuna i due testi soprattutto l'antropomorfismo spinto, che lascia trasparire dietro la bellezza della città estense quella di un «volto» (Ferrara, v. 2) femminile, immediatamente sovrapponibile al «viso di perla» della Sera (v. 15). La coincidenza, che già non è da poco, viene poi ribadita al verso 6 , dove la «sfera d'aere e d'acque» - sicuramente implicata con l'idea di Ferrara come città pregna d'acqua che emerge dai Taccuini (d'Annunzio 1965, 1976; d'ora in poi siglati rispettivamente $T$ e numero romano e $T$ e numero arabo): «I fossi dove l'acqua riluce», «I viali seguitano, molli d'acqua», «Falciano il prato subacqueo, e le erbe falciate vengono a galla», «Si sente la presenza verde dell'acqua sottostante» (T XX e XXI, passim) - evoca l'immagine dell'«acqua del cielo» (Sera, v. 17) riflessa negli occhi della donna-Sera. La prima strofe di Ferrara si chiude sul motivo della «melanconia» (v. 8), collegandosi così idealmente alla fine del brano sulle «città soavi» in Maia: «Frutti, musiche pe' nostri agi; | e le melancolie» (vv. 356-257).

L'originaria presenza delle «Grazie» in luogo delle «donne» nella redazione finale del verso 11 di Ferrara rimanda forse al taccuino appena ricordato, dove, nella descrizione di Schifanoia (T XIX, 6 novembre $1898,257)$ si apre uno spazio dedicato alle «donne»: «La vita, il movimento, la moltitudine delle donne, le eleganze, le fierezze» e subito sotto «Il gruppo delle Grazie splende su l'altura». La presenza delle donne è un motivo forte del taccuino ferrarese; se ne veda una conferma in un passo poco lontano, dedicato a Casa Romei: «Donne belle e serene, dalle belle mani mute» (T XIX, 6 novembre 1898, 259). Non è difficile leggere non già uno spunto per il componimento di Elettra, bensì le sinopie di uno dei passi più celebri di Maia, contiguo a quello sulle «città soavi» da cui siamo partiti: «Furonvi donne serene» (v. 274) e, più avanti, l'idea delle mani: «furonvi altre con mani smorte», «altre con mani esigue | e pieghevoli» (vv. 295-296).

Riprendo la lettura della seconda strofe di Ferrara proprio a partire dalla presenza femminile: «E loderò quella che più mi piacque | delle tue donne morte | e il tenue riso ond'ella mi delude | e l'alta imagine ond'io mi consolo | nella mia mente» (vv. 10-14). 
Tante donne morte nei taccuini ferraresi, ${ }^{3}$ dicevo, ma una forse si impone sulle altre: «La Certosa [...] i chiostri roggi [...] i prati pieni di tombe [...] Finalmente su una parete circondata di marmi colorati ecco la lapide nera di Marfisa a lettere d'oro» (T XIX, 6 novembre 1898, 256; il brano è contiguo ad uno sicuramente utilizzato ai vv. 19$22)$, senza dimenticare però, nel taccuino datato al giorno successivo: «Presso l'altare sono le lapidi, murate nel pavimento, con le iscrizioni funebri; di Lucrezia, di Eleonora, d'Isabella d'Este» ( $T$ XX, 7 novembre 1898, 265). Il verso 11 in prima redazione suonava «il desio che m'illude», settenario come molti altri versi del componimento, con -ude in rima con il «chiude» del verso 7, ma sottilmente implicato con un'altra delle Laudi «figlie delle acque e dei raggi», e precisamente Bocca d'Arno (del 6 luglio 1899, vv. 36-37): «la memoria | degli inganni fugaci in che s'illuse». La «favola bella» della Pioggia è ancora di là da venire, ma il tema dell'illusione è ben presente anche in queste prime prove laudistiche. Prettamente ferraresi sono i «chiostri» del verso 15, una presenza ricorrente nei Taccuini, come nella città che li ha ispirati: quelli della Certosa, di Casa Romei, del Monastero del Corpusdomini, per ricordare solo i primi che occorrono.

Nei taccuini ferraresi è però assente il Tasso (se non per un suo madrigale dedicato a Marfisa, citato di sfuggita), ${ }^{4}$ che è invece ragionevole supporre sia «l'usignolo ebro furente» dei versi 17-18. Una possibile ragione può essere indicata nella importante presenza del Tasso nella carducciana Alla città di Ferrara, apparsa nel 1898 in Rime e Ritmi, ma già stampata il 10 maggio 1895: difficile che d'Annunzio si avvicinasse di tanto, e così palesemente, a un testo altrui.

La terza strofa ci riporta di nuovo ai Taccuini, e all'intreccio con la Laus Vitae: «Loderò le tue vie piane, | grandi come fiumane, | che conducono all'infinito chi va solo [...] | e quel lor silenzio» (vv. 19-23). Lo spunto di partenza è ancora nel taccuino ferrarese: «Non ritrovano il respiro se non nel quadrivio dell'Infinito, là dove sorge il Palazzo dei Diamanti e dove le quattro strade deserte vanno ciascuna verso una meta di mistero» (T XIX, 6 novembre 1898, 253). E un passaggio de Le donne in Maia trae materia dallo stesso tassello memoriale: «Furonvi donne serene | [...] infinite | nel lor silenzio | come le contrade | piane ove scorre un fiume» (vv. 274-278); in questo caso è forse ragionevole supporre che la scrittura del testo di Elettra preceda quella di Maia (il ragionamento, se esatto, si può estendere al brano

3 E le ritroveremo nella Favilla accolta nel 1924 nel Secondo amante di Lucrezia Buti: «Quale delle donne morte mi cercherà fra i trifogli lo scongiuro di quattro foglie?» (d'Annunzio 2005, 1: 1260) e poco dopo: «Ho trascorso un pomeriggio di lugubre voluttà coi nomi delle donne estinte» (1271-2).

4 Assente anche nella Favilla, salvo il riferimento (1264) allo stesso madrigale citato nel taccuino. 
nel suo complesso). Nell'elaborazione di Ferrara, infatti, d'Annunzio esordisce con un verso lasciato in tronco «Loderò le tue», e solo in un secondo momento precisa il complemento oggetto in «vie» (che forse saranno state sin dall'inizio «piane», per via della rima baciata con «fiumane»). Sembrerebbe, ma il condizionale è d'obbligo, che il taccuino, dove è attestato il più prosaico «strade», sia stato rapidamente abbandonato, ma ancora senza un termine che lo sostituisse. Il passo di Maia, invece, è da subito lontano dal dato di realtà: le «strade» diventano prima «paesi», poi più esattamente «contrade», e soprattutto il dettato si distanzia sia dallo spunto memoriale del taccuino sia dalla sua rivisitazione poetica in Ferrara. L'Infinito, che pure nel testo di Elettra dismette la maiuscola testimoniata dal taccuino, vira decisamente per farsi attributo sia di «donne» che di «contrade»; e anche il «silenzio» ora connota le figure femminili, non più i luoghi.

Ma la filigrana del taccuino continua a essere ben presente in Ferrara: «se il fabro occulto batta su l'incude» (v. 25) non è un'immagine esornativa, come per esempio il «sogno di voluttà» del verso successivo, ma recupera, decontestualizzandola, una osservazione relativa alla Palazzina di Marfisa d'Este: «Nella Palazzina. Primo ingresso, in un cortile ove lavora un fabbro e s'ode il gemito del ferro sotto la lima» (T XIX, 6 novembre 1898, 260).

Anche Pisa, come la sorella, è fatta di poco: accorte dilatazioni di spunti memoriali, una accusata antropomorfizzazione e raffinati intrecci con le altre Laudi del 1899. L'apertura del testo, per esempio, è strettamente connessa alla prima strofa di Bocca d'Arno (composta il 6 luglio): «O Pisa, o Pisa, per la fluviale | melodia che fa sì dolce il tuo riposo | ti loderò come colui che vide | immemore del suo male | fluirti in cuore» (vv. 28-32) e nel testo che sarà poi alcyonio: "Qual donna s'abbandona | (se non tu, se non tu) sì dolcemente | come questa placata correntìa? | Ella non canta, | e pur fluisce quasi melodìa | all'amarezza. || Qual sia la sua bellezza | io non so dire, | come colui che ode | suoni dormendo e virtudi ignote | entran nel suo dormire» (vv. 6-16). ${ }^{5} \mathrm{La}$ «fluviale melodia», originariamente plurale, altro non sembra che una variazione davvero minima della «placata correntia» alcyonia che «fluisce quasi melodia», e anche l'avverbio «dolcemente» si specchia nel «dolce» «riposo» di Pisa. Quest'ultima è però acquisizione tarda, perché il verso conosce vari sviluppi: «melodie che cullano i tuoi riposi», «melodie che fan profondi i tuoi tiepidi riposi», «melodia che fa sì dolce il tuo sospiro» e infine la lezione a testo. Il «dolce» che connota il «riposo» di Pisa, oltre che con il «dolcemente» del futuro testo

5 Difficile in questo caso anche solo ipotizzare i rapporti cronologici fra i due testi; si può solo rilevare che nell'autografo custodito alla Nazionale di Roma (A.R.C.21.32/5) questi versi di Bocca d'Arno non recano in sostanza interventi d'autore (ringrazio Pietro Gibellini per l'informazione). 
di Alcyone, potrebbe essere implicato con il taccuino del 21 febbraio 1998 dedicato alla città: «E sempre, dalle finestre, davanti agli occhi, il dolce fiume» $(T 9,95)$, taccuino importante anche per altre ragioni, più solide di questa singola coincidenza.

Ma è di nuovo Bocca d'Arno che emerge con i versi successivi: «come colui che vide | immemore del suo male» di Pisa (vv. 30-31) è affine a «come colui che ode | suoni dormendo» (vv. 14-15), non solo per l'identica formulazione iniziale, ma anche per la condizione di inconsapevolezza del soggetto maschile che contempla la bellezza femminile del contesto naturale.

La conclusione della prima strofe, invece, è implicata col taccuino pisano appena ricordato: il «pianto delle stelle» del verso 35 deriva senz'altro dalla annotazione «Il cielo è purissimo, e vi rampolla il pianto delle stelle» $(T 9,95)$; solo l'aggettivo è fatto oggetto di una progressiva definizione da parte del poeta, che si muove comunque su un terreno lessicale a lui ben familiare: il «pianto» prima è «armonioso», poi l'attributo si sposta sulle «stelle» «verginali», sino all'approdo alla lezione definitiva.

Strettamente legata ai Taccuini è tutta la genesi della seconda strofe di Pisa, a partire dall'immagine femminile in apertura, che di nuovo è assunta quale equivalente della città: «Quale una donna presso il davanzale, | socchiusa i cigli, tiepida nella sua vesta» (vv. 37-38), che rimanda alla notazione, assai concreta, delle «Donne malate alle finestre» del taccuino $(T 9,95)$. La congruenza fra i due passi, pure nel tono profondamente diverso, è ribadita dal percorso variantistico, perché «il davanzale» è acquisizione più tarda di un originario «la finestra». E nella trasfigurazione onirica della città che si specchia sul fiume, l'immagine dei «santi marmi» che «ascendono leggeri» (v. 43) sviluppa una notazione ben più concreta del taccuino: «Il campanile, al tramonto, si tinge d'una delicata biondezza, diventa sì leggero che la sua inclinazione sembra dovuta allo slancio ch'egli sia per dare prendendo il vento, come un aerostato.» $(T$ 9, 94). Anche i versi immediatamente precedenti potrebbero dipendere dal taccuino, ma il rapporto è meno puntuale: «tale su le bell'acque pallido sorride | il tuo sopore» (vv. 41-42) e «l'acqua dell'Arno si fa verde. Ė immobile, come stagnante. Vi si riflettono le nuvole, i palazzi, i fanali i 3 archi del ponte» $(T$ 9, 95), o anche «L'Arno, visto dal ponte, lucido come uno specchio tenebroso: un cielo riverso.» $(T$ 9, 95).

Ultimo tassello prelavato dal taccuino pisano è quello dei versi 468: «Ma il tuo segreto è forse tra i due neri | cipressi nati dal seno | de la morte» che potrebbero essere quelli descritti nella notazione sul Camposanto: «Camposanto. 16 gennajo. Il sole illustra le finestre, le colonne sottili delle quadrifore. Nell'erba le giunchiglie gialle. I due grandi cipressi muti dalle coccole innumerevoli» (T VI, Pisa 16 gennaio 1996, 82). I versi immediatamente successivi in Pisa: «incontro alla foresta trionfale | di giovinezze e d'arbori che in festa | l'artefice creò 
su i sordi e ciechi | muri come su un ciel sereno» confermano questa ipotesi, giacché paiono con buona probabilità riconducibili alla parte in basso a destra del Trionfo della morte, la scena cortese con alberi e giovani, appunto. O invece l'assieme dei versi di Pisa, dai cipressi, ai giovani festanti, cieli compresi, potrebbe essere avvicinato alle osservazioni dannunziane sugli affreschi di Benozzo Gozzoli (che ovviamente il poeta descrive prima del loro gravissimo danneggiamento del luglio 1944): «Tutto il popolo di Benozzo a traverso le quadrifore, Benozzo solleva nei cieli verdi delle sue storie snelli alberi sovraccarichi di frutti - fra i cipressi acuti e sottili» (82) e più avanti, sempre a proposito di Benozzo: «i cani giocano, i fanciulli sorridono, i giovinetti belli portano in pugno falconi» (82).

Lo spirito di Ravenna è radicalmente diverso da quello delle prime due città: in luogo delle atmosfere oniriche che evocano gli incanti di Alcyone, troviamo una accusata dimensione eroica, ${ }^{6}$ affine certo a altri testi dannunziani, ${ }^{7}$ ma che soprattutto richiama, o anticipa, la temperie di Maia. Non la parte della Laus di cui abbiamo parlato sino a ora, fra donne serene e città soavi, ma il suo nucleo più profondo, ulissiade e dantesco. È senz'altro vero che solo per i primi 525 versi di $\mathrm{Ma}$ ia è possibile ipotizzare una data antecedente il 18 ottobre $1899{ }^{8} \mathrm{ma}$ è evidente che l'idea di rievocare in chiave mitica il viaggio in Grecia dell'estate del 1895 d'Annunzio l'avrà avuta anche prima di scrivere i versi sull'incontro con Ulisse nelle acque di Leucade (vv. 630-718). Si pensi solo all'attacco dell'episodio di Maia: «E incontrammo un Eroe», e il reiterarsi di «un altro eroe» ai versi 69 e 71 di Ravenna; e ancora il tema dell'arco, del tendere di nuovo l'arco dell'antico eroe, in $\mathrm{Ra}$ venna: «O prisca, un altro eroe tenderà l'arco | dal tuo deserto verso l'infinito» (vv. 69-70) e in Maia: «Mettimi a prova. E, se tendo | l'arco tuo grande, | qual tuo pari prendimi teco» (vv. 705-707). Certo, non tutto torna, come spesso succede all'Immaginifico, perché l'«eroe», in una variante del verso 71 detto un «vate», non è Ulisse, che neppure la sfrenata fantasia dannunziana poteva far arrivare a Ravenna,

6 Evidente sino dall'incipit «Ravenna, glauca notte rutilante d'oro» (v. 55), che allude certo al mosaico del sepolcro di Galla Placidia col suo cielo blu cosparso di stelline dorate, ma ne offre una peculiare connotazione: «sepolcro di violenti custodito | da terribili sguardi» (vv. 56-57), che si attaglierebbe senz'altro di più, dimensione cromatica a parte, al mausoleo di Teodorico.

7 Notevole, per esempio, una derivazione diretta da La nave, la prima delle Odi navali (d'Annunzio 1893), segnalata per primo da Ezio Palmieri nel suo commento alle Città del silenzio in Elettra (d'Annunzio 1943): «Va, va! Con la tua prora attingi i confini de l'acque! | Tu porti un terribile incarco. | Tutte, o Nave, le glorie degli uomini, tutte le glorie | degli uomini ne la carena | profonda con gran rombo sul gorgo oceanico porti» (vv. 11-15), che sostanzia i versi 58-62 della prima strofa dedicata a Ravenna, dopo l'allusione a Galla Placidia: «cupa carena grave d'un incarco | imperiale, ferrea, construtta | di quel ferro onde il Fato | è invincibile, spinta dal naufragio | ai confini del mondo».

8 Si veda la dimostrazione nell'Introduzione a d'Annunzio 2006. 
e quindi l'immagine dell'arco rimane, come dire, un po' spaesata, assunta più che altro in senso metaforico.

E invece Dante ad essere concretamente presente, evocato, oltre che dal ricordo implicito della sua sepoltura ravennate, dalla disseminazione della «selva selvaggia» fra i versi 67 e $68 ;{ }^{9}$ sulle sue orme il vate eroe è poi d'Annunzio stesso, ormai prossimo a dedicarsi al «suo poema» (v. 72). Un poema scritto «per vincer solo il furibondo | Mare e il ferreo Fato» (vv. 80-81), o, come leggiamo in Maia alla fine dell'episodio di Ulisse: «E io tacqui | in disparte, e fui solo; | per sempre fui solo sul Mare» (vv. 724-726). Del resto la connessione Ulisse-Dante (o meglio Ulisse-Dante-d'Annunzio) è un tema forte di Maia, sin dai testi proemiali: basta scorrere Alle Pleiadi e ai Fati, che non a caso, a lungo, si intitolò proprio Il rogo d'Odisseo, poi Il rogo d'Ulisse e solo sulle bozze arrivò al titolo definitivo.

Tutto il lessico di Ravenna è eroico e, anche in assenza di derivazioni puntuali, fa riferimento alla stessa lingua poetica di cui è intessuta Maia: si veda per esempio l'evocazione della nave, di cui abbiamo già segnalato l'origine nelle Odi navali, «cupa carena grave d'un incarco | imperiale, ferrea, construtta | di quel ferro onde il Fato | è invincibile, spinta dal naufragio | ai confini del mondo» (vv. 58-62) che andrà a riflettersi nella Preghiera a Erme di Maia, ${ }^{10}$ "O Erme, nave catafratta | or galleggia e naviga senza | vela né remi [...] | come cittadella munita, | corbame e fasciame di ferro [...] | La carena ha un cuore di fuoco» (vv. 2484-2495). O ancora i versi 66-68 di Ravenna: «Ti loderò pel mistico presagio | che è nella tua selva quando trema, | che è nella selvaggia febbre in che tu ardi» non lontani da un altro passaggio della stessa Preghiera di Maia: «L'opera attendemmo diversa, | nata da un'incognita febbre, | [...] di presagi animosa» (vv. 2807-2811).

In qualche modo, quindi, il trittico pubblicato sulla Nuova Antologia mostra una natura bifronte: contiene in nuce canzoniere e poema, e fonde temi e poetiche anche piuttosto lontani fra loro; il rapporto fra le Laudi appare dunque profondo e serrato, non solo nel fatidico passaggio fra 1902 e 1903, ma subito, sino dai primi, indimenticabili, affioramenti dei capolavori futuri.

9 «Che è nella tua selva quando trema, | che è nella selvaggia febbre in che tu ardi». 10 Il passo è sicuramente successivo alle Città del silenzio. 


\section{Bibliografia}

D’Annunzio, Gabriele (1893). Odi navali. Milano: Treves.

D’Annunzio, Gabriele (1943). Elettra. Con commento di Ezio Palmieri. Bologna: Zanichelli.

D’Annunzio, Gabriele (1965). Taccuini. A cura di Enrica Bianchetti e Roberto Forcella. Milano: Mondadori.

D’Annunzio, Gabriele (1976). Altri taccuini. A cura di Enrica Bianchetti. Milano: Mondadori.

D’Annunzio, Gabriele (1988). Alcyone. A cura di Pietro Gibellini. Milano: Mondadori.

D’Annunzio, Gabriele (2005). Prose di ricerca. 2 voll. A cura di Annamaria Andreoli e Giorgio Zanetti. Milano: Mondadori.

D’Annunzio, Gabriele (2006). Maia. A cura di Cristina Montagnani. Gardone: Il Vittoriale degli Italiani.

D’Annunzio, Gabriele (2017). Elettra. A cura di Sara Campardo. Gardone: Il Vittoriale degli Italiani.

Del Vivo, Caterina; Assirelli, Marco (1983). "Il Marzocco". Carteggi e cronache fra Ottocento e avanguardie (1887-1913). = Catalogo della mostra documentaria (Palazzo Strozzi, 19 novembre 1983-14 gennaio 1984). Firenze: Tipografia Mori.

Del Vivo, Caterina; Assirelli, Marco (1985). «Gli Orvieto: dalle prime riviste alla prima guerra mondiale». "Il Marzocco". Carteggi e cronache fra Ottocento e avanguardie (1887-1913) = Atti del seminario di studi (12-13-14 dicembre 1983), a cura di Caterina Del Vivo. Firenze: Leo S. Olschki Editore, 3-36.

Garoglio, Diego (1899). «Per le nozze di un poeta». Il Marzocco, 4(40), 2-3.

Gavazzeni, Franco (1980). Le sinopie di "Alcione". Milano; Napoli: Ricciardi.

Oliva, Gianni (a cura di) (1999). Lettere ai Treves. Milano: Garzanti. 



\section{Dignità del dolore Esercizi di consapevolezza sul Notturno di Gabriele d'Annunzio}

Federica Maria Giallombardo

Università degli Studi di Torino, Italia

Abstract Starting from the affirmations of Marziano Guglielminetti, the study intends to reread the autobiography of the Notturno by Gabriele d'Annunzio. Subsequently, It proposes an analysis on the poetics inside this peculiar literary work, in particular on the dignity of the conception of 'sorrow' that the Notturno expresses, comparing with other interpretations of 'grief' in Italian literature (Giovanni Pascoli and Guido Gozzano); and also reflecting on the d'Annunzio repertoire in general with respect to the Notturno.

Keywords Gabriele d'Annunzio. Notturno. Autobiography. Marziano Guglielminetti. Guido Gozzano. Giovanni Pascoli. Sorrow.

Sommario 1 Introduzione. Considerazioni sull'autobiografismo dannunziano. - 2 Dignità del dolore. Poetica della memoria e della sensazione. - 3 Conclusioni. Il Notturno come opera dell'impossibilità critica'.

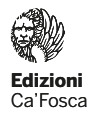

Peer review 


\section{Introduzione. Considerazioni sull'autobiografismo dannunziano}

Fino alla pubblicazione dell'accurato saggio di Marziano Guglielminetti, A chiarezza di me. D'Annunzio e le scritture dell'Io (1993), l'autobiografismo del Vate venne pressoché indistintamente interpretato, secondo i canoni del Decadentismo più struggente - per cui la convertibilità di arte e vita è una forza gravitazionale inevitabile - come forma totalizzante che culmina nell'utopia, affinché «la distanza tra manufatto artistico e realtà esistenziale possa essere soppressa e sostituita da una identificazione così assoluta da non lasciare residui» (Pupino 2002, 222; corsivo aggiunto). L'enunciazione vale pienamente, con privilegio di dibattito, per i Taccuini, definiti «libro primo della memoria» (Guglielminetti 1993, 20); e per il Solus ad solam, dove «se vita c'è, è ancora tutta affidata alla memoria» (50); ma si avverte un sottile stridore se si accostano le parole sopra citate al Notturno.

Il bisogno di verità implica in d'Annunzio la percezione e la notazione dell'istantaneo e del puntuale (lo dimostrano i circa 10.000 cartigli, strisce di carta su cui il Notturno è stato composto), riverberata nella struttura stessa (Prima, Seconda, Terza Offerta) e nella ripartizione dei chiari e delle ombre. Si afferma perciò una composizione estremamente risoluta, rendendo percepibile il dramma perpetuo della vita fisica - la lotta del giorno e della notte, parafrasando l'espressione di Victor Hugo: di tonalità cupa, ma dai colori magnifici. ${ }^{1}$ Le datazioni non scattano sotto il segno dell'epigrafe; il gusto mondano rimane sopito, sensibilizzato e annerito da svolte inattese riversate nella ricerca di suggestioni focali; ${ }^{2}$ l'ambiguità del trauma diviene strategia di comunicazione estatica; il lessico divampa qua sfuggente e là improvviso, prestigiosamente luminoso e al contempo puntuale; la tessitura vocale è ritmata, calcata, raccolta, con fiorettature e svolazzi inibiti o prosciugati. Il Vate vuole insomma dimostrare che il genio impetuoso degli artisti titanici può irradiarsi e penetrare i delicati segreti dell'anima con più consumata maestria di quanto riescano a fare coloro che eleggono la serenità della retrospettiva a territorio privilegiato della creazione. I ricordi sorgono improvvisi come oggetti saldati al buio: i periodi brevissimi sono il segno di un tono volutamente raccolto, rievocatore del passato e per questo strumento idoneo a una rilettura globale della sua vita. Decisiva l'aspirazione verso le immagini sonore in grado di cogliere una totalità, un'interez-

1 «Le relazioni sociali sono ne I Miserabili una scena nella quale, con travestimenti diversi, si rappresenta l'ancestrale lotta senza quartiere fra due principi atemporali, metafisici e religiosi: il bene e il male, Dio e il Diavolo, il cielo e l'inferno» (Vargas Ilosa 2004, 88).

2 «Ho sfiorato il prodigio con le ciglia, ho toccato il prodigio con la mano» (d'Annunzio 1921, 420). 
za, con un aggiustamento simile alla conclusione prettamente simbolista della transposition, ovvero alla selezione e all'isolamento dell'oggetto esterno trasformato per metafora o metonimia in soggetto; per il poeta si tratta di afferrare finalmente una totalità, cioè l'attimo imponderabile in cui l'estetica riunisce modelli e tensioni differenti in un unico sentimento, aspirando a una consistenza tanto naturale e perfetta da farla apparire partecipe di ognuno di essi (Debussy 1971, 46). Un esempio per tutti potrebbe essere: «Approdiamo ai Giardini per camminare un poco. Suolo duro e sonante. Alberi spogli e disperati. Se guardo i rami, mi sembra che sieno essi per spezzarsi di dolore, del mio medesimo dolore» (d'Annunzio 1921, 79-80).

Ciò che preme al d'Annunzio del Notturno è offrire l'immagine adeguata di sé e di conseguenza della propria scrittura, trafilando una sorta di 'anti-commentario' - o «commentario delle tenebre ${ }^{3}$ - ossia una rilettura globale della sua vita nel momento in cui non è vivibile, che anche nella finzione disinnescata risulti convincente, con brevitas e maestria da professionista del narrare. Per tornare all'affermazione di Pupino, citata in apertura, quella del Notturno è una rilettura che lascia sì residui: gli stratagemmi celebri e un po' consunti non si risolvono in strumenti e materiali da confessione; anzi, se è certo che alcune maschere si guastano alla luce di coscienti rivisitazioni, una pur rimane da restituire ancora e ancora, nella contraddizione di un'indole edonistica adombrata dal mistero continuo, un'attenzione «quasi miracolosa, ma anche tecnica» (Guglielminetti $1993,63)$ tipica di chi con la scrittura affida ai posteri il ritorno delle cose memorabili che lo riguardano e lo trascendono nello stesso tempo. ${ }^{4}$ Residui, in sostanza, sospesi tra il «tono dolce, dimesso, carezzevole del verso spezzato, rotto, dei periodi brevi, delle pause frequenti e stanche» (Bernini 1932, 60) del Poema Paradisiaco e il timbro «che mira alla verità, che ha il sigillo della verità» e che «deve venire dall'al di là» (Guglielminetti 1993, 51) del Solus ad solam. Si aggiunga che proprio in virtù di questi residui si precisa con straordinaria limpidità «l'idea di processo e mezzi di produzione del manufatto letterario, del 'mondo possibile', ${ }^{5}$ della 'vita superiore'» (Pu-

3 Felice definizione di d'Annunzio presente nell'Annotazione e interpretata magistralmente da Riccardi (1986, 37-9).

4 «Lo spirito umile e superbo tremava nel considerare la risma compatta e intatta da trasmutare in libro vivente» (d'Annunzio 1921, 12).

5 «Mondo possibile» che si riflette nel repertorio linguistico dannunziano. Tra la corrispondenza che reca asserzioni di lingua e di stile, impossibile tralasciare il carteggio con l'editore Treves: «Ma tu hai torto a concedermi il diritto d'inventar parole e modi. Di quel diritto io non ho mai usato. Gli italiani non vogliono ancor persuadersi che io non ho mai adoperato né foggiato un 'neologismo'. Tutte le mie parole io le ho tratte dalla più pura fonte materna, sempre [...]. Io do l'esempio del 'purismo' più rigoroso: e me ne glorio [...]. In questi tempi di futurismo, io resto fedele alla vecchia retorica dei 
pino 2002, 238) che d'Annunzio rinnova nell'opera come un atto di continua creazione.

Si è consapevoli delle parole di Gianfranco Contini, secondo cui per il «continuo e certo non accidentale passaggio dal piano letterario a quello pratico, lo schema della carriera poetica e quello strettamente biografico non possono essere separati neppure al livello più elementare» (Contini 1968, 319); altresì dell'opinione di Giorgio Bàrberi Squarotti, per il quale «raccontare la scrittura dannunziana significa raccontare anche la vita di d'Annunzio: e, naturalmente, l'affermazione vale anche all'inverso» (Bàrberi Squarotti 1992, 32); ma in questa sede l'attenzione verterà sulle differenze strutturali e etiche singolari nel genere autobiografico dannunziano, che sfidano la coincidenza di arte e vita con un invisibile sfasamento tra auto-referenzialità letteraria e referenzialità autobiografica - un apparente e premeditato scombussolamento che riporta a fuoco la vita con la lente della scrittura e che si ricalibra dialetticamente per mezzo della vita stessa. ${ }^{6}$

Due dati sono infine da richiamarsi, a dimostrazione di una stesura differente rispetto alle altre velature dell'io dell'autore. Il primo riguarda la Dedica dell'opera: «All'amore al dolore e alla morte di mia madre queste pagine scritte col sangue consacro». Come ha notato Genette, la dedica «relève [...] de la démonstration, de l'ostentation, de l'exhibition» (vedi Guglielminetti 1993, 61); ovvero «non è una dedica propriamente figliale, e quindi non favorisce una fruizione domestica del Notturno» (61), contrariamente alla fruizione professata in precedenti occasioni, ispirata ai libri di resoconti e ricordi mercantili ben noti a d'Annunzio. Colpisce che la dedica del Notturno, svilendosi, non travalichi completamente l'accezione di 'avvertenza ai lettori', tale è la sua profonda consapevole esplorazione: la madre, che dovrebbe essere ideale confidente di una scrittura definibile 'diaristica', pare trasfigurata in un correlativo oggettivo della collettività, chiamata in causa a testimoniare una stretta di mano allo specchio tra l'autore e se stesso. ${ }^{7}$

Gesuiti; e credo che l'arte letteraria ha le sue leggi e le sue regole e specialmente le sue tradizioni...» (13 aprile 1913). A tal proposito, si segnala lo studio di Caliaro (2001). 6 In una lettera al De Carolis del 10 febbraio 1917, d'Annunzio scrive: «È il diario della mia sofferenza, attraversato da un lirismo che talvolta è quasi delirio». Non a caso, Guglielminetti definì il Notturno «libro ostentatamente funereo» (Guglielminetti 1993, 60).

7 Beatrice Didier dedica un capitolo del suo journal intime a «le refuge matriciel»: «Le journal est un lieu sécurisant, c'est le refuge contre le reste de l'univers, contre ce vide, ce vertige qui risque de vous happer, contre ce saut vers l'inconnu, la multiplicité, la dispersion. L'intimité conquise, c'est l'intimité utérine et maternelle retrouvée et le recours à l'écriture pour traduire ce discours» [ «Il giornale è un luogo sicuro, è il rifugio contro il resto dell'universo, contro questo vuoto, questa vertigine che potrebbe catturarti; contro questo salto verso l'ignoto, la molteplicità, la dispersione. L'intimità conquistata è l'intimità materna uterina ritrovata, e si ricorre alla scrittura per tradurre questo discorso», Didier 1976, 91]. 
Così il primo si collega al secondo esempio: il dualismo, la frammentazione e la lacerazione di senso di errore e perdono, di tempo e soggetto, il cui globale riassestamento disilluso è il fine ultimo dell'opera. Si moltiplicano le reminiscenze colpevolizzanti e le considerazioni espiatrici. Innanzitutto, il ricordo di Beppe Miraglia, l'amico precipitato durante un volo di prova, è intenso al punto che d'Annunzio si immedesima nel suo cadavere: «Il mio compagno è morto, è sepolto, è disciolto. Io sono vivo, ma esattamente collocato nel mio buio com'egli nel suo [...]. Così la sua morte e la mia vita sono una medesima cosa» (d'Annunzio 1921, 25). L'identificazione dell'autore con l'amico - ma anche del 'poeta' con il 'soldato' - si decifra con la riflessione sul mistero di vita e morte, dicotomia divenuta inscindibile e concepita come un unico grande evento: ${ }^{8}$ «La morte è infatti presente come la vita, è calda come la vita, è bella come la vita, promettitrice, trasfigurartice» (267-8); «una tal sete di vivere è simile al bisogno di morire e di eternarsi» (267). Riguardo alla dimensione temporale, molti sono i ricordi, accanto a quello di Beppe Miraglia, dei compagni caduti: l'asse cronologico del Notturno è sicuramente quello della memoria, dove il soggetto è un centro che, a causa della cecità e dell'immobilità fisiche, si staglia e si sclerotizza nella regressione metaforica, fino al raggiungimento di senso. Difatti l'autore afferma: «solo il passato esiste, solo il passato è reale come la benda che mi fascia, è palpabile come il mio corpo in croce» (22). Dimensione tipicamente autobiografica, certamente; ma nel Notturno tempo e soggetto si confondono in un punto sinestetico che racchiude percezioni irripetibili e insieme emozioni rievocabili, come se i ricordi fossero l'«unica valvola di sfogo a tanta sensualità sotto pressione» (Parente 1992, 222). Ecco quindi che passato degli eventi e dolore del soggetto si nutrono l'uno dell'altro in maniera inedita: «I giorni d'angoscia, le notti di veglia ritornano. Il passato è presente con tutti i suoi aspetti [...]. Risoffro il mio dolore, rimpiango il mio pianto» (d'Annunzio 1921, 37); «Questa è la mia magia. Nel dolore e nelle tenebre invece di diventar più vecchio divento sempre più giovane» (229).

Date tali premesse - necessarie per comprendere la visione di chi in questa occasione scrive, lanciando un sassolino a Golia - il carattere della lettura che si appresta a esporre riguarderà un aspetto intimamente indispensabile che attraversa il Notturno: la dignità del dolore. 


\section{Dignità del dolore. Poetica della memoria e della sensazione}

È stato scritto di Debussy che la sua concezione filosofica consiste nell'idea del giorno come apice della rivelazione del segreto notturno, poiché «quando la luce è al culmine, c'è un mistero dell'evidenza suprema in cui si può leggere una profezia della notte» (Jankélévitch, Berlowitz 2012, 165); l'escatologia debussiana richiama come correlato «sia l'eternità glaciale, sia il non-essere» (Jankélévitch 1991, 39). L'aristocrazia espressiva del compositore è affine e ribaltata di segno rispetto a quella del Vate: l'immaginazione passionale ai limiti dell'audacia nel Notturno lampeggia, ferisce e acceca, insostenibilmente ricca pur senza provenire dalla consueta regione dello sfarzo cerimoniale. D'Annunzio chiama a raccolta riflessione e ingegno a alimentare il suo sforzo creativo; non tenta, però, di impadronirsi della varietà infinita di quel che splendidamente e magnificamente rileva intorno a sé, foss'anche nelle nobili e dotte combinazioni alla Tommaseo-Bellini. Nella cecità, nella mancata conquista, si apre lo spazio della poesia, del riassorbimento dei suoi tópoi: a tentoni, si cerca qualcosa che risvegli la facoltà profetica e desti il veggente nell'artista, che consenta di riappropriarsi dell'ego più profondo. Si cerca, insomma, un'arte che riemerga da qualunque patetico «indomabile dolore» (Jankélévitch 1991, 39; corsivo aggiunto).

Il dolore è identità, riconoscimento e comunicazione; un'avvolgente cognizione del limite, intrinseco all'umano, che scaturisce dal poeta e fiorisce nella bellezza. ${ }^{9} \mathrm{E}$ un'occasione di estroversione del sentimento, un'«urgenza del cuore» (Raimondi, Fenocchio 2004, 304) prima che un malessere fisico, un'esigenza di conoscere esternata spesso dall'autore: «Quando il silenzio fu fatto in me ed intorno a me, quando ebbi abbandonata la mia carne e ritrovato il mio spirito dalla prima ansia confusa risorse il bisogno di esprimere, di significare» (d'Annunzio 1921, 1). Torna insistente nel Notturno il desiderio non solo di inventare, ma appunto di significare: è nei suoi movimenti incompiuti, nelle gettate di luci e nei gesti interrotti che d'Annunzio rende la forma biologica della cecità sintomo e simbolo dell'intenso, tragico e squisito pensiero della psiche. Nel dolore «l'anima guarda il meraviglioso viso che ora è veramente il suo viso» (19). Una cristallina consapevolezza del male senza riserve permette il controllo sull'Esistenza: tutta la genealogia dell'anima - dalla concezione, di matrice euripidea e sofoclea, dell'amore come malattia; alla visione,

9 «È il mio viso, come nello specchio, come nella luce vivida, ma carico di una vecchiaia quale non patì mai alcun essere perituro. Da qual fondo di dolori e di colpe mi ritorna?» (d'Annunzio 1921, 173). 
sia poetica che medica, della melanconia secondo Galeno; ${ }^{10}$ al motivo della cecità come mancato discernimento tra realtà e apparenza, risultato artificioso dell'accurato maquillage barocco - dipende dall'onesta nozione dell'abisso, dalla realizzazione che la sofferenza sembra essere (ed è) inseparabile dall'uomo.

Dignità del dolore: nonostante sia un'esperienza comune a tutti gli individui, d'Annunzio ricerca la lirica perfetta e unica per sospingere al massimo la fantasia sensuosa, da cui la sensualità è necessariamente assente. Un'accettazione poetica che restituisce al dolore, «che ha la struttura e l'aspetto di un essere vittorioso» (118), i suoi diritti, la sua forza e la sua raffinatezza, ${ }^{11}$ costruendo e definendo l'architettura delle riflessioni dell'individuo che lo avverte, lo conosce e lo memorizza in modo soggettivo. Nel dolore, insomma, il soggetto non muta d'intensione, ma arricchisce il proprio punto di vista sull'oggetto in estensione; per questo motivo nelle fulminee intuizioni, nella rapida dizione dell'angoscia, l'anima del Notturno supera quella delle altre scritture dell'io dannunziane. Si ricorda qui una delle affermazioni testimoni: «L'aria ridiventa di cristallo gelido sotto l'acuto diamante della rapidità. Riprendo la mia corsa. Mi sembra di essere semivivo. La metà dell'anima è transita; l'altra metà è in via, è curiosa della materia di quaggiù, osserva la macchina della sua tragedia» (119). L'orizzonte del discorso è talmente annebbiato da rendere più facile avvicinarsi progressivamente alla riva che ormeggiare per asserzioni didascaliche; è sembrato quindi utile delineare il pensiero dannunziano alla base del Notturno attraverso due esempi significativi, quasi agli antipodi, che definiscono e influenzano l'atmosfera lirica novecentesca.

Emilio Cecchi scrisse che la creazione dell'identità e dell'universo poetico pascoliano tenta di soddisfare «un bisogno continuo, una fame acuta, della propria profondità interiore» (Cecchi 1912, 21). Del resto, se si annettono alcune dichiarazioni di Pascoli, come la celebre «Il ricordo è poesia, e la poesia non è se non ricordo» (Pascoli 1965, 1: 169), la tentazione di accomunare il poeta delle Myricae a

10 «Può darsi che la medicina, intesa come un insieme di scienza e arte del curare, possa identificare nel vasto terreno delle sofferenze dell'uomo la componente più conosciuta, individuabile con grande precisione e, relativamente, la più rispondente ai metodi del 'reagire' (cioè alla terapia). Tuttavia, questo è solo un aspetto della sofferenza. Il terreno della sofferenza umana è molto più vasto, e pluridimensionale: l'uomo soffre in modi diversi, non sempre contemplati dalla medicina, neanche nelle sue più avanzate ricerche» (Mercadante 2006, 8).

11 Scrive Herbert Marcuse su Freud: «La metapsicologia di Freud restituisce all'immaginazione i suoi diritti. Come processo psichico fondamentale e indipendente, la fantasia ha un proprio valore di verità, che corrisponde a un'esperienza propria, è il superamento cioè della realtà umana antagonistica. L'immaginazione tende alla riconciliazione dell'individuo col tutto, del desiderio colla realizzazione, della felicità colla ragione» (Marcuse 2001, 156-7). 
quello dell'Alcyone è comprensibile e non del tutto sconveniente. Infatti, da una parte «solo arginando il potere dell'immagine, o meglio, solo subordinandola alla sua volontà, l'autore può rintracciarsi quale soggetto nello spazio della propria rappresentazione» (Contini Bonacossi 1990, 66); dall'altra «paradossalmente, la 'perdita' [...] non è tanto quella, esplicitamente dichiarata dal poeta, del passato [...] ma viceversa, quella della individuale, attuale, identità del soggetto e della sua immagine che, ricercata [...] viene perennemente occultata, schermata, dalla insistenza con cui si presentano i ricordi» (67). Tuttavia, se per Pascoli sono proprio le immagini mnestiche, rievocate, a ostacolare il recupero sulla pagina e a garantire il moto vitalistico di permanente desiderio della loro effettiva rappresentazione (la cosiddetta dynamis della poesia pascoliana), per il d'Annunzio del Notturno l'immagine del poeta nell'opera non protegge la realtà dell'io dalla sua ontologica infermità e dalla sua misera finitezza. Il dolore non si plasma, ma si attraversa pateticamente e si estende a partire dal poeta stesso; esso è 'tutto e sempre', e l'opera non può 'schermare', superare o negare un male segreto che segreto non è più, che si manifesta e che condiziona il pensiero: pertanto il Notturno raggiunge una catarsi superiore a quella delle tragedie dannunziane. ${ }^{12}$ Inoltre, la consapevolezza dell'autore non coincide con la risposta vitale a fondamento del cristianesimo 'pascoliano',13 «considerato essenzialmente come religione sacrificale e caritativa, capace di tramutare il dolore in amore e di vincere l'arcaica soggezione istintuale all'aggressività» (Gioanola 2000, 75); piuttosto, è un nuovo mito, «il più bello» (d'Annunzio 1921, 19). Non si può contraddire il limite della realtà, nemmeno con gli stratagemmi fantasmatici forniti dalla scrittura: nell'infermità pesa ciò che non si è esperito quanto ciò che si è vissuto; ha importanza tanto chi si è perduto quanto chi riceverà testimonianza della conversione da «lungo dolore» (19) ad «apice di purità» (19). ${ }^{14}$

12 D'Annunzio ha penetrato l'animo umano come un grande drammaturgo, imparando a conoscere le sue gioie e i suoi dolori, la sua esultanza e la sua disperazione; rendendo ogni rappresentazione della passione umana. Per approfondimenti, si segnala l'Introduzione di Donato Pirovano alla Francesca da Rimini (d'Annunzio 2018, 7-18).

13 Elio Gioanola afferma che «Pascoli sente forte, lui agnostico, il fascino del sacerdozio» poiché «è lui stesso un prete, avendo preso i voti, forzosi, di castità e obbedienza». Ragionamento all'opposto quello del Notturno, che fa apparire Pascoli «un pessimo D'Annunzio o un Marinetti ante-litteram [...] con il flavour tutto suo di un sentimentalismo da brividi» (Gioanola 2000, 279).

14 Tornano alla mente, allora, le parole di Goethe nella Zueignung (Dedica) del Faust, precisamente nella seconda e terza strofa (Goethe 2004, 1: 2-3): «Ihr bringt mit euch die Bilder froher Tage, | Und manche liebe Schatten steigen auf; | Gleich einer alten, halbverklungnen Sage | Kommt erste Lieb und Freundschaft mit herauf; | Der Schmerz wird neu, es wiederholt die Klage | Des Lebens labyrinthisch irren Lauf | Und nennt die Guten, die, um schöne Stunden | Vom Glück getäuscht, vor mir hinweggeschwunden. | Sie hören nicht die folgenden Gesänge, | Die Seelen, denen ich die ersten sang; | Zer- 
Di matrice schopenhaueriana, l'impossibilità di districare il nesso tra rappresentante e rappresentato nella poesia di Guido Gozzano mette in discussione il rapporto tra verità e finzione, vita e letteratura. Come per d'Annunzio, anche secondo l'autore piemontese scrivere è «entrare nello spazio narcisistico» (Contini Bonacossi 1990, 80) e ambivalente della raffigurazione; ma il raggiungimento dello statuto dell'opera d'arte autoreferenziale è conseguito con evoluzioni opposte. Gozzano non può che smarrirsi nell'ambivalenza intrinseca della parola, nell'alienazione della soggettività nonché nella specularità della propria scrittura, che coincide sia con il sogno che con il ricordo - si legge nella poesia La via del rifugio: «Non agogno | che la virtù del sogno: | l'inconsapevolezza» (Gozzano 1983, 70). Inconsapevolezza come «unica tregua concessa all'Io nei confronti dell'eterno affermarsi del presente» (Contini Bonacossi 1990, 80) e, all'opposto, consapevolezza come «eterno affermarsi della morte, che priva il soggetto della volontà e della possibilità di agire» (81). In d'Annunzio il gioco temerario dell'immaginazione, che mira al sublime nelle regioni sovrasensuali, non parte da un oggetto ma dal soggetto che, in una sorta di camera oscura mentale, ha rifratto il ricordo della visione di tale oggetto; in altre parole, la contemplazione non è dell'avvenimento in sé, benché esso sia traumatizzante e attiri molte energie. ${ }^{15}$ Allo stesso modo in cui un colorista smorza tutti i contrasti inquietanti con sfumature intermedie, le scintille lacrimose del Notturno vengono filtrate e nebulizzate dall'osservazione della sensazione che l'avvenimento procura: «Nel mio occhio piagato si rifucina tutta la materia della mia vita, tutta la somma della mia conoscenza. Esso è abitato da un fuoco evocatore, continuamente in travaglio» (d'Annunzio 1921, 22); «Il mio dolore riceve, aggira e rapisce come un vortice le sue parole misurate» (63). Entrambi gli autori fanno derivare, in un modo o nell'altro, il presente della loro scrittura dal passato;

stoben ist das freundliche Gedränge, | Verklungen, ach! Der erste Widerklang. | Mein Leid ertönt der unbekannten Menge, | Ihr Beifall selbst macht meinem Herzen bang,| Und was sich sonst an meinem Lied erfreuet, | Wenn es noch lebt, irrt in der Welt zerstreuet» («Vi seguono immagini di giorni sereni e più di una cara ombra sale su con voi. Simili ad una vecchia e svanita saga, risorgono in me e il primo amore e l'amicizia. Il dolore si rinnovella, il rimpianto ripercorre l'errabondo e labirintico corso della vita e enumera i nomi dei buoni che il destino defraudò delle ore felici e sono scomparsi prima di me. Le anime per le quali cantai i primi canti, non udiranno i nuovi che seguiranno; lo stuolo degli amici è disperso, spenta ne è, ahimè, la prima eco. Il mio dolore si espande verso una folla ignota; persino il suo applauso opprime il mio cuore, e quelli che, un tempo, si rallegravano del mio canto, se ancora vivono, errano dispersi per il mondo»). Si potrebbe ingenuamente dedurre che il tema del Notturno sia un calzante prosieguo della melanconia romantica; ma l'idea di meditazione della e nella convalescenza dannunziana poco concorda con il motivo vorticoso dello scoramento, con la fiammante apparizione famelica e con la teutonica Sehnsucht.

15 «Nuova nozione del tempo. Lotta fra l'immagine viva, continuamente creata dal ricordo, e il corpo immobile» (d'Annunzio 1921, 64). 
ma Gozzano distrae l'Io dal presente dell'esistenza e la sua scrittura è «passiva riproposizione di una realtà solo osservata» (Contini Bonacossi 1990, 82). Il riflesso dell'immagine di Gozzano è sfuggente; la sua parola specchiata è insieme illusione e menzogna. L'immagine di d'Annunzio, invece, si rifiuta di essere intimidita da qualunque difficoltà di un'autentica rappresentazione ideale. Sia Gozzano che d'Annunzio affidano la propria effige di autore «a un futuro che la conserva intera oltre la morte» (96); ma la salvaguardia nel presente dall'opera di decadenza e di vanificazione, che la vita inevitabilmente impone, dal primo è rifiutata mentre dal secondo è tenacemente conquistata. Entrambi fondamentalmente credono nell'immodificabilità del tempo e in un passato vividissimo; ma il dolore di Gozzano è sterile e afferma l'assenza del desiderio proprio perché tutto proiettato nel passato, in una realtà romanzesca e ironica; rappresenta il Vivente nella morte, il presente verso la morte. Il dolore di d'Annunzio è, invece, un'epifania della morte; esso abita e anima naturalmente, con perpetua agnizione, il Vivente. Il tempo del dolore di d'Annunzio è formato da sincopi, è quello della dignitas; quello di Gozzano, è il tempo zero, o tempo della miseria. ${ }^{16}$

\section{Conclusioni. Il Notturno come opera dell'‘impossibilità critica'}

In questa occasione si è tentato di tracciare alcune linee di un bozzetto irrimediabilmente incompleto: non a caso, tra i maggiori studiosi dell'autore, pochi recentemente hanno dedicato studi di ampio respiro al Notturno. ${ }^{17}$ La scelta non è solamente dettata da ragioni di capacità interpretativa o di mero gusto stilistico: l'opera dannunziana in esame vanifica in ogni riga, a ogni inciampo, se stessa. ${ }^{18} \mathrm{Il}$ suo meccanismo interno, sulla soglia del prototipo, risulta sorprendente rispetto alle altre letture dannunziane e rende il Notturno opera dell'impossibilità critica', speculare alla biografia dell'autore e perciò, nella sua inafferrabilità, magneticamente affascinante (nonostante i voli alle volte troppo ardimentosi).

D’Annunzio non era soddisfatto se non sforzandosi di soggiogare qualunque disposizione d'animo a una copia fedele della sua idea poetica: il Notturno invece mostra una fase più rischiosa, in cui il puro

16 Interessante, a tal proposito, lo studio di Meroi (2018).

17 Fra i critici e storici della letteratura italiana che hanno trattato l'argomento spicca, per competenza e gentilezza di espressione, Carla Riccardi (Riccardi 1986, 1988), che ha pubblicato vari saggi in preparazione dell'edizione critica del Notturno per l'Edizione Nazionale delle Opere di Gabriele d'Annunzio.

18 «L'anima attonita allude a sé stessa» (d'Annunzio 1921, 316). 
potere creativo subordina il genio, richiedendo l'assistenza talvolta di uno sforzo stupefacente, di un'autentica rivelazione da parte del lettore. Ravvisare la manifestazione del miracolo quotidiano dell'eccezionale bagliore della notte paga, insomma, il prezzo dell'anomalia. ${ }^{19}$ Come il più moderno degli uomini e ormai come il più nobile, il Vate non concepisce il potere del male in sembianze ripugnanti; bensì in un'ottica salvifica e di redenzione laica, mai devozionale.

Nel breve e assolutamente non esaustivo studio proposto, la concentrazione, allorché didascalica, è stata sempre guidata da una passionale aderenza al tema della dignità del dolore - da esercizi di consapevolezza che si eseguono tra le pieghe dell'indole e che chi legge impara dalle mosse meditative dell'autore. L'educazione alla sofferenza, intesa come comprensione culturale dei limiti naturali e rivalutazione del fallimento in quanto occasione di affinamento di prospettiva, è un obiettivo principe nella contemporanea formazione sentimentale; un argomento che oggi, in un'epoca di superomismo estenuante, di prepotenza e di rifiuto della fragilità umana, proprio l'opera di un d'Annunzio frainteso potrebbe aiutare a risanare e esorcizzare.

\section{Bibliografia}

\section{Opere letterarie}

D’Annunzio, Gabriele (2018). Francesca da Rimini. A cura di Donato Pirovano. Roma: Salerno.

D’Annunzio, Gabriele (1921). Notturno. Milano: Treves.

Debussy, Claude (1971). Monsieur Croche et autres écrits. Paris: Gallimard.

Goethe, Johann Wolfgang (2004). Faust e Urfaust, vol. 1. Traduzione di Giovanni Vittorio Amoretti. Milano: Feltrinelli.

Gozzano, Guido (1983). Tutte le poesie. Milano: Mondadori.

Pascoli, Giovanni (1965). Tutte le opere di Giovanni Pascoli, vol. 1. Milano: Mondadori.

\section{Opere critiche}

Anglani, Bartolo (1996). I letti di Procuste. Teorie e storie dell'autobiografia. Roma-Bari: Laterza.

Bàrberi Squarotti, Giorgio (1992). La scrittura verso il nulla. D’Annunzio. Torino: Genesi.

Battistini, Andrea (1990). Lo specchio di Dedalo. Autobiografia e biografia. Bologna: il Mulino.

Bernini, Ferruccio (1932). Commento alle Poesie liriche di Gabriele D’Annunzio. Bologna: Zanichelli.

19 «V'è un luogo dell'anima, là dove il nero fiume e il fiume chiaro confluiscono [...]. Le nostre immagini vi si rispecchiano e vi si confondono» (27) 
Bigi, Emilio (1967). Le genesi del Canto notturno e altri studi su Leopardi. Palermo: Manfredi.

Caliaro, Ilvano (2001). L'amorosa guerra. Aspetti e momenti del rapporto Gabriele D’Annunzio-Emilio Treves. Venezia: Istituto Veneto di Scienze, Lettere ed Arti.

Caputo, Rino; Monaco, Matteo (a cura di) (1997). Scrivere la propria vita. L'autobiografia come problema critico e teorico. Roma: Bulzoni Editore.

Cecchi, Emilio (1912). La poesia di Giovanni Pascoli. Napoli: Ricciardi.

Compagnon, Antoine (2000). Il demone della teoria. Letteratura e senso comune. Torino: Einaudi.

Contini, Gianfranco (1968). Letteratura dell'Italia unita. 1861-1968. Firenze: Sansoni.

Contini Bonacossi, Valentina (1990). La parola di Narciso. Roma: Bulzoni Editore.

Didier, Béatrice (1976). Le journal intime. Paris: PUF.

Gioanola, Elio (2000). Giovanni Pascoli. Sentimenti filiali di un parricida. Milano: Jaca Book.

Guglielminetti, Marziano (1993). A chiarezza di me. D’Annunzio e le scritture dell'lo. Milano: FrancoAngeli.

Jankélévitch, Vladimir; Berlowitz, Béatrice (2012). Da qualche parte nell'incompiuto. Traduzione di Valeria Zini. Torino: Einaudi.

Jankélévitch, Vladimir (1991). Debussy e il mistero. Traduzione di Carlo Migliaccio. Bologna: il Mulino.

Marcuse, Herbert (2001). Eros e civiltà. Traduzione di Lorenzo Bassi. Torino: Einaudi.

Mercadante, Silvia (2006). Il dolore. Valutazione, diagnosi e trattamento. Milano: Masson.

Meroi, Fabrizio (2018). Tra miseria e dignitas. Immagini della follia da Alberti a Voltaire. Pisa: Edizioni ETS.

Parente, Rosa (1992). «Colori al buio. Appunti sul codice cromatico del Notturno». Oliva, Gianni (a cura di), D’Annunzio. Per una grammatica dei sensi. Chieti: Solfanelli, 221-8.

Pupino, Angelo Raffaele (2002). D’Annunzio. Letteratura e vita. Roma: Salerno Editrice.

Raimondi, Ezio; Fenocchio, Gabriella (2004). Da Pascoli a Montale. Vol. 1 di La letteratura italiana. Il Novecento. Milano: Mondadori.

Riccardi, Carla (1986). «L'elaborazione del Notturno. Il delirio lirico organizzato». D'Annunzio notturno = Atti dell'VIII Convegno di Studi dannunziani (Pescara, 8-10 ottobre 1986). Pescara: Fabiani, 37-61.

Riccardi, Carla (1988). «La prima forma del Notturno». Paragone-Letteratura. Firenze: Mandragora, 24-31.

Vargas Ilosa, Mario (2004). La tentazione dell'impossibile. Victor Hugo e "I Miserabili". Milano: Scheiwiller. 


\title{
La memoria 'breve' delle Faville del maglio
}

\author{
Raffaella Bertazzoli \\ Università degli Studi di Verona, Italia
}

\begin{abstract}
The present work traces an hermeneutic line between the poem «La morte del cervo" in Alcyone and some Faville del maglio, prose texts in which d'Annunzio experiences a form of autobiography and the celebration of poetic self. Starting from the Favilla, dated 1907, celebrating the sculpture of Clemente Origo, this essay establishes links with some texts, written much later, in which the poet turns poetic creation into a mythical 'event'. In the last favilla, the symbolic act of the poet, who becomes a centaur, can be read allegorically as a poetic act.
\end{abstract}

Keywords Faville. Event. Myth. Enthousiasmos. Poetry.

Sommario 1 Vivo, scrivo. - 2 La parola in tre dimensioni. - 3 «Trasumanar significar per verba». -4 II mito vissuto.

$\begin{array}{lll} & \text { Peer review } & \\ & \text { Submitted } & 2019-02-04 \\ \text { Edizioni } & \text { Accepted } & 2019-05-28 \\ \text { Ca'Foscari } & \text { Published } & 2019-10-15 \\ & \text { Open access } & \end{array}$

(C) 2019 | (a) Creative Commons Attribution 4.0 International Public License 
Vaincus, mais non domptés, exilés, mais vivants, Et malgré les édits de l'Homme et ses menaces, Ils n'ont point abdiqué, crispant leurs mains tenaces Sur des tronçons de sceptre, et rôdent dans les vents. (Paul Verlaine, Les Dieux, vv. 1-4)

\section{Vivo, scrivo}

Citata come dichiarazione della genesi delle Faville, l'intervista del 4 giugno 1909, rilasciata da d'Annunzio a La Tribuna, contiene un importante cambio di passo, dando inizio a una nuova forma di prosa, stilisticamente scarna, legata all'impressione estemporanea e alla ricerca del 'tempo perduto'. Gli abbozzi, stesi come bacino memoriale e in forma ancillare per l'elaborazione di altre opere, diventano autonoma materia poetica:

Spessissimo, a ogni disegno che mi sorge nell'animo, butto giù frammenti, abbozzi di scene, ecc. E in riguardo a tutti questi frammenti di cose rimaste allo stato di disegno, ho con Treves l'impegno di un'opera dal titolo Le faville del maglio in cui faccio la storia delle cose che non ho scritte, e che verrà a essere come una specie di mia storia interiore, un edificio documentale del mio spirito e del mio istinto. (d'Annunzio 2003, 2: 1433)

Il primo nucleo di faville (Faville del maglio-Memoranda), scritte da d'Annunzio in Francia, nel suo rifugio di Arcachon, affacciato sull'Atlantico, venne consegnato alle pagine del Corriere di Luigi Albertini tra il 1911 e il $1914 .{ }^{1}$ Sono prose elaborate dai Taccuini, ma con una fisionomia autonoma, tutta dentro uno stretto giro retorico. Il riaffiorare del passato si articola nelle forme di un diario intimo, dove l'introspezione diviene visionaria e sciolta spesso dal mero dato temporale. Le faville, scrive d'Annunzio in una lettera a Emilio Treves del 19 marzo 1913, sono «saggi d'analisi interiore, a cui il luogo e la data non sono se non pretesti» (d'Annunzio 1999b, 474; corsivi nell'originale). Le Faville-Memoranda rappresentano, in fase embrionale, l'anticipazione di quella diffusa e ininterrotta linea autobiografica che diviene caratteristica ineludibile del d'Annunzio 'notturno'. ${ }^{2}$

1 Per i dati filologici e un'attenta analisi critico-stilistica si veda il saggio di Martignoni 1987, 63-81; per le dinamiche testuali del passaggio delle Faville dal Corriere della sera al volume, tra il 1924 e il 1929, cf. Martignoni 1977, 308-53; Martignoni 2014, 65-75.

2 Andreoli scrive che nelle Faville: «deflagra un narcisismo che vede nell'autobiografia lo sbocco obbligato. Non più una serie discontinua di 'apparizioni' [...] al passato si ritorna con l'intento di cogliervi i 'segni dell'alta sorte', il presagio di un destino d'eccellenza» (d'Annunzio 2005, 2: 3321-2). 
Da questi primi lacerti di memoria, scritti per il Corriere, d'Annunzio passa negli anni del Vittoriale a un disegno editoriale organico. Interviene in profondità a riscrivere la materia estemporanea, facendo dilagare il dato personale. Nell'Avvertimento preposto al Venturiero senza ventura e altri studi del vivere inimitabile (1924), il poeta giustifica come atto di «superbia» la raccolta delle sue «prose [...] le più ardite, arditamente estratte dal libro della mia memoria» (d'Annunzio 2005, 1071). Si tratta del più appariscente e narcisistico atto di autocelebrazione che il poeta compie sotto l'urgenza creativa: «Sono, nel tempo medesimo, beato e disperato. M'è impossibile di arrestare la vena» (d'Annunzio 1999b, 718-19). ${ }^{3}$ Nel Libro segreto (1935), avrebbe riconosciuto ancora una volta l'ambigua dualità del suo carattere, agonisticamente attratto verso l'oscuro territorio dell'invasamento. La «vena», di cui diceva all'editore, attinge alla manìa, la finzione poetica dell'artefice 'mostro' in agone con l'ordine del logos:

pur nella più tenue e nella più potente ode di Alcyone non è tanto mistero quanto nei numeri della mia prosa recente; ove io aduno gli arcani della Magìa e quelli della Poesia non dissimili. [...] Postero di me medesimo confido ai posteri candidi o torbidi queste due formule. E séguito a vivere, studiosamente voluttuosamente sprezzantemente: nel tempo medesimo più mostruoso del mostro e lineare come la perfezione. (d'Annunzio 2005, 1878-9)

I ricordi, le riflessioni, gli stati d'animo stesi da d'Annunzio, oltre alle prose del Venturiero senza ventura andranno a ingrossare il volume del Compagno dagli occhi senza cigli (1928), sorta di anamnesi testamentaria della propria esistenza. ${ }^{4} \mathrm{Al}$ centro è sempre il soggetto che ritrova nella memoria i segni della predestinazione. Faville dell'ego di un artista del «vivere inimitabile» che attinge linfa nell'indissolubile ebbrezza esistenziale e creativa. Le descrizioni meta-letterarie, riferite alle opere composte o da scrivere, traggono forza da un vitalismo che era stato, a suo tempo, celebrato nei grandi libri delle Laudi: eroe prescelto per un contatto diretto con il mito, dove l'esperienza immaginifica lambisce i contorni del reale. L'io eroico viene rappresentato nell'atto sublime, l'io erotico nel pieno dell'esperienza sensuale. Vivere e scrivere si sovrappongono e si fondono:

3 La lettera del 4 giugno 1924 è indirizzata a Guido Treves.

4 Le Faville del maglio, dopo una pubblicazione parziale in riviste, uscirono in due tomi. (T. 1, Il venturiero senza ventura e altri studii del vivere inimitabile; T. 2, Il compagno dagli occhi senza cigli e altri studii del vivere inimitabile). Il progetto di un terzo tomo, che si sarebbe dovuto intitolare La serva meschinella dal gran cuore e altri studii del vivere inimitabile, non si realizzò; un gruppo di Faville, denominate involate, furono stampate sul Secolo XX il 20 ottobre del 1928. 
Vivo, scrivo. Le mie vene pulsano, i miei polmoni respirano, la mia penna scorre, nello stesso mistero continuo, nello stesso prodigio misurato, nel medesimo gioco inimitabile. Scrivere è per me il bisogno di rivelarmi, il bisogno di risonare, non dissimile al bisogno di respirare, di palpitare, di camminare incontro all'ignoto nelle vie della terra. Scrivere è per me obbedire alla legge profonda dell'essere. (d'Annunzio 2005, 1428)

Partendo dalle forme d'inventio visionaria, presenti nell'alcionia Morte del cervo, e al commento della favilla intitolata La risurrezione del centauro (1907), prenderemo in considerazione altri due testi, scritti molto più tardi e inseriti in luoghi contigui nel Secondo amante di Lucrezia Buti: L'ommorto e il centauro e Il dèmone mimetico (1924). Nostro intento è di analizzare il rapporto che esiste tra il testo poetico, composto il 24 agosto 1902 a mezzanotte, e le faville, che riconducono l'esperienza visionaria del testimone alcionio alla più intima e interna storia dell'io. Seguendo le coordinate di alcuni lavori che indagano il rapporto tra mito e modernità, proviamo a tracciare una linea interpretativa tra il testo poetico e le faville, in un décalage di esperienza interiore. Cercheremo di capire come d'Annunzio traduca l'inverarsi del mito nella scrittura prosastica, sia come espressione di ermeneutica poetica sia come lacerto di manìa creativa.

\section{La parola in tre dimensioni}

La favilla La resurrezione del centauro, datata 1907, nasce come commento alla scultura di bronzo di Clemente Origo, intitolata La morte del cervo, presentata alla VII Esposizione internazionale di Venezia. ${ }^{5}$ L'opera è dedicata all'omonima lirica dannunziana di Alcyone. Si tratta di uno scritto con il taglio di una breve prosa saggistica, il cui valore simbolico si traduce nel rapporto tra la natura plastica della parola poetica e l'aspetto tridimensionale dell'opera scultorea, espressione assoluta dell'apollineo. ${ }^{6}$ Nell'explicit, il concetto prende forma nella retorica del chiasmo:

5 La prosa, con il titolo «Le forze e le forme. La resurrezione del centauro» esce sul Corriere della Sera il 21 aprile 1907; poi, nello stesso anno, in opuscolo (Roma, Staderini) con il titolo definitivo e accompagnato dalla lirica alcionia La morte del cervo. Il testo in 99 esemplari è impreziosito dalle xilografie di Adolfo de Carolis (cf. Martignoni 1987, 310). La favilla viene poi accolta definitivamente in volume.

6 Sulla natura plastica della parola, d'Annunzio cita il volgarizzamento trecentesco delle Metamorfosi di Arrigo Simintendi, che legge nell'edizione del 1850. Il testo è presente nella biblioteca del Vittoriale ed è abbondantemente segnato. Sul Marzocco del 14 gennaio 1906 compare «L'encomio di bronzo», posto nel volume a seguito della «Resurrezione del centauro». L'esperienza della colata di bronzo richiama volutamente il luogo della Vita del Cellini in cui si parla della fusione del Perseo. 
Qui veramente la parola è formata di tre dimensioni. E qui si vede come veramente tutte le arti, quando sviluppano la massima energia espressiva, si riducano a quella 'unità ritmica' che abolisce il mezzo materiale. L'arte dà la qualità alla materia, non la materia all'arte. Come il verbo perde la sua inconsistenza, così il bronzo perde la sua fissità. L'imagine statica e l'imagine dinamica non sono create se non da due ordini di ritmi puri. (d'Annunzio 2005, 1579)

Descrivendo la fusione del gruppo statuario del centauro in lotta con il cervo, d'Annunzio rinvia al suo testo poetico, nato dal sentimento del thaumàzein, dallo stupore/terrore provato assistendo alla scena della lotta tra la figura mitica del centauro e un cervo, sulle rive del fiume Serchio. Il tempo e lo spazio sono annullati nel «grande tempo mitico», che Roger Caillois dice «situato nel contempo all'inizio e al di fuori dell'avvenire» (Callois 2001, 99). L'apparizione, colta nell'attimo del kairòs, è subito svanita nella favola: «Sparve Ombra labile | verso il Mito nell'ombre del crepuscolo» (d'Annunzio 1995, 280, vv. 159-160).

Nella Morte del cervo, d'Annunzio fissa l'evento', il modo in cui il divino si manifesta agli uomini come 'evidenza'. Lo spazio del simbolo estetico diventa cifra dell'artifex additus artifici, la cui critica non razionalistica del mito diventa espressione 'salvifica' del mito.

Siamo nel tempo ciclico delle epifanie, in cui l'origine sacra della poesia si fa fondamento ontologico in grado di esprimere ciò che il linguaggio metafisico non è più in grado di nominare. Un evento epifanico che per Károly Kerényi «può essere concepito in greco anche così: quando esso accade è dio» $(2001,107) .^{8}$

Nella riscoperta del fondamento tragico della cultura, Aby Warbug parla della percezione degli dèi come di un'«onda mnemica» che «dalla regione del passato» lo investe (Warbug 1984, 46). Roberto Calasso ricorda (citando l'Odissea) che «Non a tutti appaiono gli déi in piena evidenza». E prosegue istituendo un rapporto tra epifania e poesia: "Enargés è il terminus technicus dell'epifania divina: aggettivo che contiene in sé il bagliore del 'bianco', argós, ma finirà per designare una pura indubitabile 'evidenza'. Quella specie di 'evidenza' che poi verrà ereditata dalla poesia. Ed è forse il

7 «Ogni spirito vivente compie ancora oggi e sempre compirà in futuro l'atto al quale gli dèi delle religioni debbono la loro esistenza; la differenza è soltanto che le creazioni della nostra fantasia per noi non sono più esseri reali. Tanto meno si può negare al vero artista e poeta così come al fruitore assorto la forza di riunire ancora una volta le figure non più credute del mito storico, di ripetere l'atto già eseguito della loro creazione». (Vischer 1922, 435)

8 Elabora il tema delle varie forme del riconoscimento in letteratura, il volume di Boitani (2014). 
tratto che la differenzia da ogni altra forma» (Calasso 2001, 17). ${ }^{9}$

Le riflessioni di Kerényi e di Calasso richiamano il pensiero di Heidegger per il ruolo fondante che il filosofo assegna al poeta. Nel saggio «Wozu Dichter?» a commento dell'elegia Brot und Wein di Hölderlin, Heidegger elabora il concetto di viaggio nel profondo, spazio dove è possibile seguire le tracce degli dèi fuggiti. In queste regioni della memoria sommersa, il pensare poetante della denkende Dichtung di Hölderlin è in grado di ritrovare i vestigi degli dèi che hanno abbandonato il mondo. Nella loro prossimità con il divino, i poeti «più arrischianti», come Hölderlin, «dicono il taciuto», rendono ragione del Sacro nel tempo della povertà:

I più arrischianti sono coloro che nella mancanza di salvezza si rendono conto del nostro essere-senza-protezione. Essi apportano ai mortali la traccia degli Dei fuggiti nelle tenebre della notte del mondo. I più arrischianti, in quanto cantori della salvezza, sono «poeti nel tempo della povertà». (Heidegger 1982, 296)

Tutta la poesia di Hölderlin, scrive Mittner, è una «teofania vespertina» (Mittner 1971, 713). Per uscire da questa «notte del mondo», è necessario che il poeta intraprenda questo viaggio nella regione dell'Offenbarkeit della rivelazione dell'essere, cogliendo gli dèi nel pieno della loro «evidenza».

Ma torniamo a d'Annunzio. L'autobiografismo del poeta-testimone dell'«evidenza», nei cui versi si era formalizzata la 'bestialità' del mito, cede la parola all'artifex che sorveglia nella favilla il circolo ermeneutico: «Tuttavia quel centauro apparso nella pineta tirrena non era un'idea esemplare ma era un vero corpo bimembre» (d'Annunzio 2005, 1578).

Dichiarata la natura dell'essere biforme, apparso all'improvviso nel fiume alle ombre del crepuscolo, d'Annunzio passa alla sua descrizione:

Mezzo uomo mezzo cavallo ma non mostro appariva l'ospite della selva estiva; ché in quella parte ove l'uomo era commesso al cavallo un miracolo assiduo di vita si compieva senza discordanza, con la perfezione dell'innesto superando la bellezza dell'una e dell'altra forma distinte. (d'Annunzio 2005, 1576) ${ }^{10}$

Sono riprese le tessere lessicali del XII libro ovidiano delle Metamorfosi, che avevano fatto da tessuto al testo poetico. La fonte classica è accolta da d’Annunzio a due livelli: è il luogo della meraviglia per

9 Sul 'mito' del Rinascimento tra Warburg, Pater, d'Annunzio, si veda la tesi di Brugnara 2010.

10 Sulla fenomenologia del metamorfico in d'Annunzio cf. Balducci 1989. 
la prodigiosa vicenda metamorfica che investe tutte le cose; ma anche il luogo della violenza ermeneutica e della sovra-interpretazione. L'uso della fonte diviene suggestione creativa e cifra del pathos che la riscrittura forza nel senso di un processo continuo di riprese e di rimaneggiamenti.

La figura altera del centauro è forgiata sulla doppia connotazione mitica di bestialità e di sapienza, descritta come: «una sola volontà di rapina e di conoscenza» (d'Annunzio 2005, 1576). ${ }^{11}$ Il centauro, modello di naturalità e forza primigenia, vestigio di un tempo, fuori del tempo, è il mito più vicino all'uomo moderno in cui convivono le due forze creative del dionisiaco e dell'apollineo, dell'istinto e della techne:

Fraterna tra tutte le creature generate dal suolo mitico! Nessuna ci tocca, anche oggi, più a dentro; nessuna ci sembra meglio rappresentare la più recente delle aspirazioni umane, meglio significare il nuovo aspetto della vita terrestre, poiché l'uomo moderno non è se non un centauro storpio e mutilato il quale ricostituisce il mito primitivo riconnettendo indissolubilmente il suo genio all'energia atroce della Natura. (d'Annunzio 2005, 1: 1576-7)

L'uomo moderno è inverato nel centauro dannunziano che cerca di riconnettersi alla dualità costitutiva dell'essere, riportandolo alle sue energie primordiali: modo simbolico di vivere il mito come possanza istintuale del corpo e abilità della tecnica.

Il mito eclissato non è estinto: può rivivere come forma possibile nell'opera del poeta, nel 'pensare poetante' (dichtendes Denken) che va in cerca delle tracce degli dèi.

Nella parola immaginale si realizza la rinascita del Sacro, si dà forma alla dimora degli dèi, accogliendo il senso del linguaggio originario che per Heidegger è un ritorno al «gioco di pensiero [...] più vincolante del rigore della scienza» (Heidegger 1973, 1: 104-5):

Allora il testimone di tanto spettacolo cercò di foggiare un suo poema in una massa di materia ritmica, giusta la simiglianza dei due esseri vivi; e operando riconobbe l'identità della sua arte poetica

11 Probabile l'influenza di Guérin, Le Centaure: «un jour que je suivais une vallée où s'engagent peu les centaures, je découvris un homme qui côtoyait le fleuve sur la rive contraire. C'était le premier qui s'offrît à ma vue, je le méprisai. Voilà tout au plus, me dis-je, la moitié de mon être ! Que ses pas sont courts et sa démarche malaisée ! Ses yeux semblent mesurer l'espace avec tristesse. Sans doute c'est un centaure renversé par les dieux et qu'ils ont réduit à se traîner ainsi» (de Guérin 1840, 506). Qualche nesso intertestuale è riscontrabile con Le vase di Henri de Régnier (Jeux rustiques et divins): «Une autre fois, | un centaure passa la rivière à la nage, | l'eau ruisselait sur sa peau d'homme et son pelage; | il s'avança de quelques pas dans les roseaux, | flaira le vent, hennit, repassa l'eau; | le lendemain, j'ai vu l'ongle de ses sabots | marqué dans l’herbe...» (de Régnier 1897, 117). 
con l'arte plastica cui tendeva il suo sforzo di rilievo e di saldezza. (d'Annunzio 2005, 1: 1578)

\section{3 «Trasumanar significar per verba»}

Nella favilla L'ommorto e il centauro (Secondo amante di Lucrezia Buti, con data fittizia 1907), d'Annunzio ci riporta al 1902, all'estate di Romena, nel Casentino, la grande stagione delle Laudi. L'idea del poiéin si condensa nel momento delle «metamorfosi immortali», sempre al limite della sperimentazione. La favilla ci immette nel tempo 'festivo', dove la vita reale urge nell'allegoria della creazione poetica. D’Annunzio si fa evento e con questa sensazione forte crea poesia: «La colata è di sangue bono; e ne' rami di gitto, nei numeri del verso, mi discende dal cervello. Tanto fervore, tanto vigore, tanto furore [...] Dentro ci voglio ritrovare il dio vero» (d'Annunzio 2005, 1221).

In questa ricerca, come dirà poco più avanti, il creatore sottrae l'esistenza all'errore del tempo:

Ero al colmo della vita [...] Rimemorare non è per me aver vissuto né rivivere; ma è vivere nel vivere. [...] nel senso del potere in ogni attimo dal vertice abbracciare tutto il giro di essa vita. [...] Io vivo, a me sempre piacque di vivere, su l'orlo del rischio e su l'orlo del segreto. Su l'uno e su l'altro è intieramente abolito il comun senso del tempo. Su l'uno e su l'altro come sul vertice, non vige se non quella specie di tempo che è la fluidità stessa della vita interiore. (d'Annunzio 2005, 1224)

In più passaggi del suo saggio, Kerényi (2014) riflette sulla necessità per l'uomo moderno di rifondare il rapporto (Umgang) col Sacro. Attraverso il dialogo, che egli intrattiene con Jung, istituisce un collegamento tra mitologia e psicologia del profondo, riandando a quel tempo primordiale che è la vera sorgente del 'mito genuino'. La festa e l'enthousiasmos sono esperienze connesse.

Sulla linea di Jung, James Hillman (1977) vede nell'altrove, nella regione della nostra psiche, il luogo dal quale affiora la narrazione mitica come archetipo dell'anima: «Soltanto quando il mito è ricondotto nell'anima, soltanto quando il mito assume importanza psicologica diviene una realtà vivente, necessaria per la vita, e cessa d'essere un artificio letterario, filosofico o religioso» (Hillman 1977, 29; corsivi nell'originale). Seguendo la «via psichica al mito», l'uomo moderno esce «completamente dal pensiero temporale e dalla storicità e [si muove] verso una regione immaginale, un differenziato arcipelago di ubicazioni, dove gli Dei sono, e non quando essi furono o saranno» (16; corsivi nell'originale). 
Come nella lirica di Alcyone, nella favilla d'Annunzio fa dell'evento l'essenza della 'festa':

balzato in sella, aderito al mantel sauro, sùbito mi sentii mezzo uomo e mezzo cavallo, sùbito si sentì egli mezzo cavallo e mezzo uomo. [...] Ero un cavaliere? Ero un formatore? Formavo me stesso dal limo della terra? Rimpastavo il mio limo e ne facevo una figura incognita? Veramente m'innestavo nella bestia? Imparavo l'arte degli innesti dall'inventore greco del centauro? [...] mi godo d'una specie di fervore muscolare, d'una sorta di ebrezza 'scùltile o conflàtile', ponendomi in un accordo equestre così perfetto che quasi è come s'io mi provassi in commettermi al cavallo e in ottener la perfezione dell'innesto. (d'Annunzio 2005, 1220-1)

Scrive Kerényi che la poesia stessa è 'evento' festivo «proprio in quanto è arte autentica e pura, e possiede realmente un carattere creativo» (Kerényi 2001, 100).

In quella stessa stagione, rimemorata nella favilla (siamo nel 1902), d'Annunzio concepisce il disegno di una tragedia intitolata Cola di Rienzi. ${ }^{12}$ Il Proemio, originariamente succinto, viene dilatato nell'edizione del 1912, assecondando quella tensione autobiografica che è cifra caratterizzante delle prose, a partire da quegli anni. Il passo è nel segno dell'imbestiare elevato al significato di «trasumanare» con riferimenti precisi ai testi alcionii:

Anche là, in quella stalla chiusa, tutto era lontananze e apparizioni dello spirito, tutto era disegni e scritture dello spirito, azioni mutue tra me e gli iddii subitanei. Anche là sentivo il mio cuore divenire più profondo e il mio occhio riacquistare la limpidità infantile [...] come al Gombo, come nella Versilia, come quando nasceva dal mio respiro Undulna. Era ben là Undulna, trasfigurata in una grande cavalla baia che meritava il nome della pieghevole dea «dai pie d'ali»; [...] ma per entro i suoi belli occhi biechi scoprivo l'essere sconosciuto e divino che mi spiava come io un giorno tra le canne del Serchio spiavo il Centauro. [...] Hai tu mai pensato che imbestiare può in un certo senso essere un modo di trasumanare? [...] Vedo che il mio segreto lirico è in una sensualità rapita fuor de' sensi. (d'Annunzio 1999a, 17-8)

La sperimentazione del «trasumanare» ha un suo altissimo vertice lirico nel Meriggio (composto tra il luglio e l'agosto 1902). Il testo

12 La stesura finale prende la forma di una biografia quella che, preceduta da un breve Proemio, vede la luce in tre puntate sul Rinascimento (1 e 15 dicembre 1905, 5 gennaio 1906). 
poetico è una delle espressioni più intense della 'evidenza' del divino. Nell'ora della liturgia pànica, nel tempo delle epifanie divine, in uno scenario immerso nel silenzio e nell'immobilità assoluti, il poeta sperimenta la perdita della propria identità umana. Annullandosi nella Natura smarrisce la propria individualità:

E non ho più nome.

E l'alpi e l'isole e i golfi

e i capi e i fari e i boschi

e le foci ch'io nomai

non han più l'usato nome

che suona in labbra umane.

Non ho più nome né sorte

tra gli uomini; ma il mio nome

è Meriggio. In tutto io vivo

tacito come la Morte.

E la mia vita è divina.

(d'Annunzio 1995, 150-1, vv. 99-109)

I riferimenti a Nietzsche sono evidenti, per quella capacità del superuomo d'indiarsi nella Natura al di là di ogni limite umano, entrando nel flusso dell'esistenza. Per Nietzsche: «Sotto l'incantesimo del dionisiaco non solo si restringe il legame fra uomo e uomo, ma anche la natura estraniata, ostile e soggiogata celebra di nuovo la sua festa di riconciliazione col suo figlio perduto, l'uomo» (Nietzsche 1972, 26).

Questo rapporto stretto con il Sacro viene interpretato da Hillman nel segno dell'ineluttabilità: «non possiamo toccare il mito senza che ci tocchi a sua volta» (Hillman 1977, 30).

\section{$4 \quad$ Il mito vissuto}

Nel Dèmone mimetico, d'Annunzio va oltre i confini del testo scritto e si fa protagonista del mito in un processo di costante e inesauribile ripresa dell'atto poematico. Da creatore di metamorfosi diviene soggetto metamorfico che supera il limite umano, rappresentandosi. ${ }^{13}$ Il demone dannunziano si rivela anche nei termini dell'ethos anthropoi daimon di Eraclito, che Giorgio Colli interpreta, traducendo: «la propria qualità interiore, per l'uomo, è un dèmone» (Colli 1993, 103).

Nella favilla, l'accadimento divino si manifesta nel non-tempo, dove gli dèi appaiono, dove si diventa dèi. Soffermandosi sulla polarità e sulla presenza di modelli archetipici junghiani, James Hillman

13 Un saggio interpretativo, a ampio raggio, sul rapporto tra d'Annunzio, scrittura saggistica e ideologia si trova in Lonardi 1988, 77-96. 
commenta i due estremi del comportamento istintuale:

nell'uno abbiamo un modello di comportamento coatto e arcaico: nell'altro le immagini archetipiche. Sicché l'istinto agisce e nello stesso forma un'immagine della sua azione. Le immagini fanno scattare le azioni: le azioni sono modellate sulle immagini. Perciò ogni trasformazione delle immagini incide sui modelli di comportamento, talché ciò che facciamo nella nostra immaginazione possiede rilevanza istintuale. [...] Agendo sull'immaginazione, partecipiamo alla natura 'dentro di noi'. (Hillman 1977, 62-3)

D’Annunzio si rappresenta in una posizione agonale, nella furia che spezza la 'penna-freccia'. La poesia nasce dalla radice magica di una dea-maga di metamorfosi: Circe. La finzione/immaginazione (l'immaginale di Hillman) si manifesta nelle forme di manìa creativa, di delirio panico. D’Annunzio ne riconosce la valenza fino al limite e mimesis è allo stesso tempo poiésis, crezione e poesia.

La scena che d'Annunzio mostra al lettore/spettatore può trovare una sua possibile ermeneutica in alcuni passi dei dialoghi platonici che definiscono la funzione del poeta dentro il concetto di enthousiasmos. Condizione che lo porta a 'indiarsi', a essere entheos (riempito dal dio). Platone intende per follia divina quella del poeta preso da invasamento, il quale scopre in sé improvvise energie creative. La sua poesia è dono divino. Entusiasmo, dunque, è manìa poetica:

l'invasamento e la mania che provengono dalle Muse, impossessatesi di un'anima tenera e pura, la destano e la traggono fuori di sé nell'ispirazione bacchica in canti e in altre poesie, e, rendono onore a innumerevoli opere degli antichi, istruiscono i posteri. Ma chi giunge alle porte della poesia senza la mania delle Muse, pensando che potrà essere buon poeta in conseguenza dell'arte, resta incompleto, e la poesia di chi rimane in senno viene oscurata da quella di coloro che sono posseduti da mania. (Platone 1998, 245a)

Nel Dèmone mimetico, protagonista è il corpo che si manifesta nel dionisiaco nietzschiano dell'annullamento dell'identità, del principium individuationis, entrando nel flusso continuo, nel ciclo inarrestabile della Natura primigenia (Veronesi 2003):

Ora m'imbestio. Di non so che divina bestialità m'inebrio, e di non so che semifera poesia pasturata dell'erbe di Circe, magata di pastura favolosa. Ora la finzione è demenza; e di abbandonarmi alla mia demenza pànica ho una tema puerile. (d'Annunzio 2005, 1222)

Nella Repubblica (10: 617e), Platone dice che «Non sarà il demone a scegliere voi, ma sceglierete voi il vostro demone». In particola- 
re per il culto di Dioniso, Platone parla dell'atto mimetico come processo di alienazione che consente agli iniziati di identificarsi con gli dèi. Il poeta, posseduto dal demone, dice Platone, cade in uno stato di alienazione mentale che lo porta a superare l'intelletto e la techne, collegandolo alla sfera irrazionale dove la creazione è dono divino.

Nella «qualità della [...] finzione materiata», il poeta entra nello stato mentale di estasi, quella in cui si percepisce di avere «un dio dentro di sé». L'imbestiamento, che porta al doppio mostruoso, significa delirio imitativo:

Che più m'importa della mia arte se io stesso divengo la materia della mia arte, la qualità della mia finzione materiata? [...] Una specie di demone mimetico mi possiede. La sua veemenza mi respinge dalle mie carte, mi prende, mi tiene. Mi preme la nuca, mi piega la schiena, m’abbassa le braccia, mi ponta le mani aperte su l'impiantito di mattoni, mi cangia le mani e i piedi in quattro zoccoli, m'avviluppa la lingua tra parola che rigna e nitrito che parla, mi chiude nel contorno vocale della mia strofe che io chiudo. (d’Annunzio 2005, 1222-3)

Scrive Warburg che: «L'ethos apollineo germoglia insieme con il pathos dionisiaco quasi come un duplice ramo da un medesimo tronco radicato nelle misteriose profondità della terramadre greca» (Warburg, 1980, 306-7).

Misterioso, mostruoso, divino: sono le contraddizioni connotative di Dioniso, che Giorgio Colli vede

nell'immediatezza, nell'interiorità di un cacciatore che si slancia spietato e di una preda che sanguina e muore, tutto ciò vissuto assieme, senza prima né dopo, e con pienezza sconvolgente in ogni estremo [...] Nel crearlo l'uomo è stato trascinato a esprimere se stesso, tutto se stesso, e qualcosa ancora al di là di sé. Dioniso non è un uomo: è un animale e assieme un dio, così manifestando i punti terminali delle opposizioni che l'uomo porta in sé. Qui appunto sta l’origine oscura della sapienza. (Colli 1995, 15)

Per Massimo Fusillo, l'esperienza dionisiaca si caratterizza per l'ibridazione tra pensiero (logos) e pathos, portando alla destabilizzazione. L'esperienza dionisiaca implica uscire dai limiti della propria persona, dalla nozione d'identità individuale verso l'alterità:

Il nucleo profondo del mondo dionisiaco è proprio la coesistenza fra l'esplosione violenta della passione e la sua ricodificazione: fra il caos e il ritorno all'ordine, fra il magma e la sua espressione, fra i linguaggi non verbali del corpo, della musica, dello sguardo, e le strategie della retorica. (Fusillo 2006, 8) 
Ragionando sulla categoria estetica del dionisismo, Massimo Fusillo scrive che: «emozione e pensiero sono due logiche che si ibridano di continuo fra di loro, e che hanno bisogno l'una dell'altro per potersi esprimere e per potersi alimentare» (Fusillo 2006, 8). Nella favilla, il linguaggio non verbale del corpo si fonde nell'ibridare: «la lingua tra parola che rigna e nitrito che parla» (d'Annunzio 2005, 1: 1222). In questo mimetismo, l'evento si fa parola poetica che 'significa'. D'Annunzio si rappresenta nel testo, «nel contorno vocale della mia strofe che io chiudo. - Ma l'uom co' pugni avea divaricato $\mid e$ divelto le corna del nemico» (1222; corsivi nell'originale).

Nell'immagine plastica il termine rivelatore è «mistero», che il poeta colloca nel «fondo dei tempi», in cui la possessione è la forma di conoscenza più rischiosa. Il rito violato, scrive d'Annunzio, espone a un «attimo indicibile di vergogna e quasi di schianto, che mi rapisce a patire nel fondo dei tempi il sacrilegio del mistero interrotto, del segreto violato» (d'Annunzio 2005, 1223). La favilla si conclude nella rappresentazione della fisicità spaesata dell'invasamento, mentre lo stato di spossessamento circoscrive l'evento, si scioglie nel rito della festa:

mi rotolo, mi rannicchio, mi nascondo il viso tra le braccia; ricopro la mia confusione e il mio rossore; mi sembra d'esser ferito, mi sembra d'esser blandito; non so s'io abbia voglia di piangere, s'io abbia voglia di ridere. [...] non so se mi convenga di ostinarmi nel mio cruccio puerile o di lasciarmi sciogliere dalla grazia festevole. (d'Annunzio 2005, 1: 1223)

Alla conclusione della favilla il poeta scrive: «Non so più nulla di me, non so più nulla del mio prodigio né del mio delirio» (d'Annunzio 2005, 1: 1223).

Parecchi anni dopo, ritornando nel Libro segreto sulla potenza dell'eros, d'Annunzio riconosce la compresenza di umano e divino, d'istintualità e mistero nelle forze che governano l'esistenza: «la bestia è una forma del divino, anzi il più misterioso aspetto del divino» (1864). Imbestiare è dunque anche una forma del trasumanare. 


\section{Bibliografia}

Balducci, Marino Alberto (1989). Il sorriso di Ermes. Studio sul metamorfismo dannunziano. Firenze: Vallecchi.

Boitani, Piero (2014). Riconoscere è un dio. Scene e temi del riconoscimento nella letteratura. Torino: Einaudi.

Brugnara, Gabriella (2010). 'Un'imprecisione simile al sogno': La Ninfa nell'opera di Gabriele d'Annunzio e nella cultura del suo tempo [PhD dissertation]. Trento: Università degli Studi di Trento.

Caillois, Rager (2001). L'uomo e il sacro. Trad. di Laura Guarino; a cura di Ugo M. Olivieri. Torino: Bollati Boringhieri.

Calasso, Roberto (2001). La letteratura e gli dèi. Milano: Adelphi.

Colli, Giorgio (1995). La sapienza greca, vol. 1. Milano: Adelphi.

d'Annunzio, Gabriele (2005). Alcione. A cura di Pietro Gibellini, pref. e note di Ilvano Caliaro. Torino: Enaudi.

d'Annunzio, Gabriele (1999a). La vita di Cola di Rienzo. A cura di Pietro Gibellini; note di Maria Pertile, Milano: Mondadori.

d'Annunzio, Gabriele (1999b). Lettere ai Treves. A cura di Gianni Oliva. Milano: Garzanti.

d'Annunzio, Gabriele (2003). Scritti giornalistici. A cura di Annamaria Andreoli e Giorgio Zanetti, Milano: Mondadori.

d'Annunzio, Gabriele (2005). Prose di ricerca, vol. 1. A cura di Annamaria Andreoli e Giorgio Zanetti. Milano: Mondadori.

de Guérin, Maurice (1840). «Le Centaure». Revue des Deux Mondes, 15 de mai, 506-9.

de Régnier, Henri (1897). Jeux rustiques et divins. Paris: Société de Mercure de France.

Fusillo, Massimo (2006). Il Dio ibrido-Dioniso e le «Baccanti» nel Novecento. Bologna: il Mulino.

Heidegger, Martin (1982). Sentieri interrotti. Trad. di Pietro Chiodi. Firenze: La Nuova Italia.

Heidegger, Martin (1973). In cammino verso il linguaggio. Milano: Mursia.

Hillman, James (1977). Saggio su Pan. Milano: Adelphi.

Kerényi, Károly (2001). Religione antica. Milano: Adelphi.

Kerényi, Károly (2014). Il rapporto con il divino. A cura di Fabio Cicero. Milano: Bompiani.

Lonardi, Gilberto (1988). Alcibiade e il suo demone. Parabole del moderno tra d'Annunzio e Pirandello. Verona: Essedue.

Martignoni, Clelia (1977). «Sull'elaborazione delle "Faville del maglio"». D’Annunzio, il testo e la sua elaborazione. Quaderni del Vittoriale, 5-6, ottobre-dicembre, 308-53.

Martignoni, Clelia (1987). «Le prime "Faville del maglio" (1911-1913)». D’Annunzio Notturno. Pescara: Centro Nazionale di Studi Dannunziani, 63-81.

Martignoni, Clelia (2014). «Intorno alle Faville del maglio e alla sperimentazione diaristica». Gabriele d'Annunzio 150. "Vivo, scrivo". A cura di Giordano Bruno Guerri. Milano: Silvana Editore, 65-75.

Mittner, Ladislao (1971). Storia della letteratura tedesca. Torino: Einaudi.

Nietzsche, Friedrich (1972). La nascita della tragedia. Trad. di Sossio Giametta; a cura di Giorgio Colli. Milano: Adelphi.

Platone (1998). Fedro. A cura di Giovanni Reale. Milano: Bompiani. 
Raffaella Bertazzoli

La memoria 'breve' delle Faville del maglio

Platone (2001). Ione. Prefazione, saggio introduttivo, trad., note e apparati di Giovanni Reale. Milano: Bompiani.

Veronesi, Matteo (2006). Il critico come artista dall'estetismo agli ermetici. d'Annunzio, Croce, Serra, Luzi e altri, vol. 3. Bologna: Azeta Fastpress.

Vischer, Friedrich Theodor (1922). «Das Symbol». Kritische Gänge, vol. 4. A cura di Robert Vischer. München: Meyer und Jessen, 420-56.

Warburg, Aby (1980). La rinascita del paganesimo antico. Trad. di Elio Cantimori. Firenze: La Nuova Italia.

Warburg, Aby (1984). «Burckhardt e Nietzsche». Trad. di Roberto Calasso. Aut aut. Rivista di filosofia e cultura, 199-200, 46-9. 

Civiltà dannunziana 



\title{
L'eredità di Ovidio in Giovanni Pascoli e Gabriele d'Annunzio
}

\author{
Maria Belponer \\ Università Ca' Foscari Venezia, Italia
}

Abstract The contribution analyses the influence of the Ovidian work, especially the Metamorphoses, on Giovanni Pascoli and Gabriele d'Annunzio, emphasising how the ancient poet is understood in different ways. In the diversity of interpretation and reformulation of the myths of the Latin poet, both Pascoli and d'Annunzio come to the creation of narratives and figures that reflect central aspects of their poetic world, often far from the original inspiration.

Keywords Myth. Allusion. Mythopoeia. Sources. 
Nella prefazione ai Poemi conviviali, dedicati a Adolfo De Bosis, Giovanni Pascoli ricorda l'invito che aveva ricevuto da De Bosis stesso e da Gabriele d'Annunzio a condividere l'esperienza del Convito, rivista di breve vita, che nasce nel 1895, anno intenso per Pascoli per molte ragioni, prima fra tutte l'elaborazione e la pubblicazione, appunto nel Convito, di Solon, il primo dei Poemi conviviali. In quella prefazione Pascoli menziona d'Annunzio con l'invocazione «O mio fratello, minore e maggiore, Gabriele»: minore d'età, otto anni di meno, e maggiore di fama, concessione che Pascoli fa volentieri, data la vicenda artistica già matura di d'Annunzio, a confronto con la sua (e la prefazione stessa innesta qualche elemento di sofferenza da questo punto di vista, per esempio nel riferimento alla Minerva oscura, opera dalla sorte infelice, che getta addirittura un'ombra sui Conviviali).

Basterebbe questa evocazione a giustificare l'associazione dei due poeti, quasi rituale negli studi su quel periodo, ma c'è molto altro: per esempio le vicende, testimoniate dalle apparizioni letterarie e dall'epistolario, che, nonostante le fratture, corrono parallele. E del resto lo stesso d'Annunzio dedica il suo Alcyone a Pascoli, nel congedo del Commiato, in cui l'amico/avversario poeta, definito «figlio di Virgilio», è rappresentato mentre «ad un cipresso [...] tacito siede». Soprattutto c'è una modalità parallela nel leggere il mondo antico, con scelte diverse e una differenza di sguardo e di voce, che si coglie anche nel rapporto con Ovidio, a costruire due mondi dissimili, pur nell'utilizzo non così divergente della fonte.

Se è vero che «Il mito, in Ovidio, è il campo di tensione in cui [...] forze si scontrano e si bilanciano» (Calvino 1979, 9), proprio questa percezione del mito come campo di tensione vale per Pascoli come per d'Annunzio, così come vale l'idea di un sistema di allusioni intrecciate: il modello cui entrambi si riferiscono, quando ricorrono alla modalità allusiva $o$ alla ricodificazione di un mito, non è mai solo Ovidio, ma per esempio sono Ovidio e Omero, Ovidio e Dante; per entrambi la realizzazione poetica della lettura delle fonti è la capacità di evitare il centone, il mosaico realizzato con le pietruzze antiche: è l'espressione di un rapporto del poeta col mondo antico e di una sua idea originale.

Al primo sentore, per così dire, Ovidio non sembra essere un autore che possa rientrare nella sensibilità pascoliana: egli non è tra quelli studiati nelle antologie scolastiche, alcune delle quali costituiscono l'unica testimonianza della filologia pascoliana; o meglio, il progetto di occuparsi della poesia elegiaca, e dunque di Ovidio, resta allo stato di ideazione nella collana Nostrae litterae che Pascoli voleva dedicare alla Scuola classica, di cui comparvero solo il sesto e il primo volume, rispettivamente Lyra, dedicata alla poesia lirica, ed Epos, all'epica. Infatti, nonostante la «Nota» premessa a Lyra, a partire dall'edizione 1899, affermi: «il secondo [volume], in due sezioni, conterrà nella prima sezione gli Epyllia e gli Idyllia, nella seconda una larga scelta delle Metamorfosi (sic) di Ovidio», a questo progetto non fu dato se- 
guito, ma della «Nota» resta preziosa l'indicazione delle Metamorfosi come opera di riferimento per l'esemplificazione della poesia ovidiana, e quest'opera comparirà, in tracce di diversa natura e ampiezza, nei Poemi Conviviali.

La presenza di Ovidio nei Conviviali è riscontrabile in diverse forme, che vanno dalla disseminazione allusiva, intesa come inserimento di un tassello testuale circoscritto, che rimanda a un significato più ampio in rapporto al testo cui si allude, alla riproposizione e ricodificazione di un mito ovidiano, alla creazione di miti metamorfici, sulla scorta dell'ideazione delle Metamorfosi stesse.

Nel secondo dei Conviviali, «Il cieco di Chio», il poeta divenuto cieco è accomunato all'alcione:

Né già la bianca tua beltà celasti

a gli occhi della sua memore mano:

non vista ad altri, che a lui cieco e, forse,

al solitario tacito alcïone.

(Pascoli 2009, 29, vv. 15-18)

La giovane ninfa Deliàs rivela la sua bellezza alle mani del poeta, che suppliscono alla cecità dei suoi occhi, e all'alcione, uccello marino frutto della metamorfosi della sposa di Ceice, Alcione appunto, trasformata in uccello in seguito al lungo dolore per l'assenza e la morte del marito (Ov., Metamorfosi, XI, passim). L'allusione coglie la consonanza della situazione tra il poeta e Alcione: la frustrazione della giovane sposa si riflette in quella del poeta, destinato a immaginare la bellezza della ninfa senza poterla cogliere, mentre percepisce per la prima volta il limite connesso alla sua condizione, analogamente ad Alcione, che deve infine rassegnarsi alla perdita del marito.

Una lettura in un certo senso rovesciata del testo ovidiano si riscontra in un passo dell'Ultimo viaggio, nel poema VII, «La zattera» :

E l'uomo allora udì di contro un canto

di torte conche, e divinò che dietro

quelle il nemico, il truce dio del mare, venìa tornando ai suoi cerulei campi.

Lui vide, e rise il dio con uno schianto

secco di tuono che rimbombò tetro;

e venne.

(Pascoli 2009, 124, vv. 270-277)

L'espressione «torte conche» traduce quasi letteralmente cava bucina tortilis, con cui, in Ovidio (Met., I, vv. 335-336), Tritone placa il mare per ordine di Posidone, ma qui esse vengono attribuite a Posidone, «il truce dio del mare», che solleva la tempesta contro Odisseo, con un richiamo a Odissea, V, v. 291, al quale si aggiunge l'enig- 
matico riso del dio nei confronti di Odisseo che sogna di riprendere la navigazione.

La riproposizione di miti ampiamente narrati nelle Metamorfosi assume diverse forme, a partire dalla combinazione, per così dire, delle allusioni ai miti di Procne e Filomela, evocati nel «Poeta degli Iloti», a sua volta elaborato sulla traccia dell'Agone di Omero ed Esiodo, composto su materiale antico e tramandato nei Corpora esiodei e omerici. Il racconto anonimo narra che, nonostante il successo più evidente di Omero, «il re incoronò vincitore Esiodo sostenendo che era giusto che vincesse colui che esortava all'agricoltura e alla pace e non colui che narrava di guerra e di stragi» (Magugliani 1979, 219) secondo una tendenza evolutiva dalla poesia epica a quella didascalica che rappresenta anche la rinnovata ispirazione morale della poesia, profondamente condivisa da Pascoli, che riporta la notizia dell'agone in Epos, XXIII, nel quale traduce parte del testo. Tuttavia nel «Poeta degli Iloti» la scelta della poesia agreste in luogo di quella eroica è una svolta segnata dall'incontro con lo schiavo, dal quale nasce l'esaltazione del lavoro umano come fonte di progresso e di autorealizzazione, tema anche questo profondamente esiodeo e pascoliano. In questo contesto tematico, l'inserto del mito di Procne e Filomela è riportato alla lettura che ne dà lo schiavo, che attribuisce il canto della rondine all'alacrità del risveglio al mattino, e alla gioia della sera, quando avverte le sue faccende opportunamente compiute. Nell'interpretazione dello schiavo, la rondine e l'usignolo sono personificati e rappresentano l'una lui stesso, l'altro il poeta: il canto di entrambi non è pianto, ma gioia e piacere del canto e del lavoro compiuto, nell'allusione simbolica ai valori fondamentali: lavoro e poesia, modello alternativo al motto di Sileno: ${ }^{1}$

\author{
E il buon Ascreo diceva: «Ecco, fu tolto \\ il sonno, tutto al querulo usignolo ${ }^{2}$ \\ che così piange per la notte intiera, \\ né sotto l'ala mai nasconde il capo; \\ ma solo mezzo, a quella cui la sera \\ gemere ascolta e riascolta l'alba. \\ Miseri! e un solo è il lor dolore e forse
}

1 Il motto di Sileno, che costituisce un motivo ricorrente nei Conviviali, è evocato, nell'Agone di Omero ed Esiodo, da Omero, il quale sostiene che per un uomo il bene più grande è la morte, 0 , una volta nati, morire il più presto possibile.

2 Allusione al mito di Procne e Filomela, ampliata in seguito con l'inserimento della rondine; il canto dell'usignolo è lamentoso, poiché, secondo la versione ovidiana del mito, (Met., VI, vv. 421 e ss.) rappresenta la metamorfosi di Filomela che, insieme a Procne, la rondine, piangono la tecnofagia di Iti; il mito è narrato anche da Eliano (Varia Historia, XII, v. 20), che attribuisce le caratteristiche di questi uccelli a Esiodo. Si veda su questo Petrarca (Canzoniere, CCCX, v. 3): «et garrir Progne et pianger Philomena», anch'esso presente in questo poema (cf. v. 30, garriva). 
l'uno non ode mai dell'altro il pianto!»

E lo schiavo diceva: «Oh! non è pianto questo né l'altro. Ma la casereccia rondine ha molti i figli e le faccende, e sa che l'alba è un terzo di giornata; e dolce a quegli che operò nel giorno, viene la sera, e lieto suona il canto dopo il lavoro. E l'usignol gorgheggia tutta la notte né vuol prender sonno... ch'egli non vuole seppellir nel sonno, avere in vano dentro sé non vuole un solo trillo di quel suo dolce inno.

(Pascoli 2009, «Poeta degli Iloti», vv. 276-294)

Analogamente, il mito di Psiche, narrato nei Poemi di Psyche, vede l'intreccio tra le Metamorfosi di Apuleio e quelle Ovidio, nell'allusione alla vicenda di Pan che soffre per l'amore infelice nei confronti di Siringa, come Psiche piange per Eros; ancora una volta, la lettura pascoliana fa registrare una reinterpretazione che trae origine dalla sovrapposizione di diverse fonti principali e dalle allusioni, disseminate nel testo, ad altre secondarie: il pianto di Pan diviene canto d'amore e si riflette nel pianto di Psiche:

Tu piangi, quando Pan, la notte, fa dolcemente sufolar le canne; ${ }^{3}$ piangi d'amore, o solitaria Psyche, nella tua casa, dove più non tieni posto, che l'ombra, e non fai più rumore, che l'alito

[...]

E Pan allora un dolce canto soffiò nelle palustri canne, che tu piangesti a quel pensier di morte come piangevi per desìo d'amore.

(Pascoli 2009, 246-7, vv. 45-50; 250-1, vv. 104-107)

Un significativo esempio di riproposizione e ricodificazione di un mito è costituito dal poema «Le Memnonidi», il IV dei Conviviali, pubblicato per la prima volta in Atene e Roma, nel marzo del 1904. Il poema è strettamente connesso con il precedente, «La cetra d'Achille»: Achille si era addormentato aspettando l'Aurora, tenendo tra le braccia Briseide nella sua ultima notte, e ora Aurora sorge a portare l'ul-

3 Tu piangi... canne: Pan soffre per l'infelice sorte di Siringa, secondo la narrazione di Ovidio, Met., I, vv. 687-712. 
timo giorno all'eroe. Il racconto si svolge nell'arco di tempo dall'alba al sorgere del sole, quando inizia il nuovo giorno di guerra. All'eroe acheo è legata la vicenda di Memnone, uccisore di Antiloco, che aveva recato per primo al Pelide la notizia della morte di Patroclo: Memnone, infatti, figlio di Aurora, sarà ucciso da Achille, che con questo gesto si avvicina ormai definitivamente al suo destino di morte. Aurora, io narrante del poema, celebra i due giovani, che ama entrambi, l'uno nel ricordo della giovinezza e nella profezia della prossima fine, l'altro nella rievocazione della morte recente, legata al mito, narrato da Ovidio, secondo cui dal suo rogo nacquero, per concessione di Zeus alla madre, le gralle Memnonidi, uccelli palustri che ogni anno inscenano un duello sulla tomba di Memnone.

Tuttavia, mentre nel racconto ovidiano (Ov., Met., XIII, vv. 576 e ss.) lo scontro è cruento, nella rivisitazione pascoliana esso è del tutto innocuo e diviene una sorta di danza commemorativa degli antichi duelli eroici. Su questa struttura narrativa si intrecciano altri echi: la leggenda dei Colossi di Memnone, statue innalzate dai Tolomei sul presunto luogo della tomba di Memnone, da una delle quali scaturiva un lamento, dovuto probabilmente a una fessurazione, in cui il poeta legge il pianto dell'eroe, affine a quello della madre (Ov., Met., XIII, v. 622), rappresentato dalla guazza mattutina, e al lamento di Achille, quando incontra Odisseo nell'Ade. ${ }^{4}$ Aurora descrive con accenti delicati la danza delle Memnonidi e lo sguardo di Memnone che la vede ridare luce al mondo, con un'espressione di stampo omerico, «la mia mano [...] rosea», del tutto consona all'intonazione epica del testo:

E quando io sorgo, le Memnonie gralle fanno lor giochi, quali intorno un rogo, non come aurighi con Ferèe cavalle sbalzano in alto sotto il lieve giogo, con la lucida sferza su le spalle;

e né come unti lottatori ignudi che si serrano a modo di due travi e né come aspri pugili coi crudi cesti allacciati intorno ai pugni gravi ma come eroi, con l'aste e con gli scudi

Quasi al fuoco d'un rogo, al mio barlume ecco ogni eroe contro un eroe si slancia:

4 Odissea, XI, vv. 477 e ss.; l'episodio è qui riproposto con un significativo ampliamento del racconto, dove la prospettiva di una vita che sconfessa l'eroismo dell'eroe dell'Iliade, che pure appartiene alla narrazione dell'Odissea, giunge a vagheggiare una vita rurale, che assume i tratti del mondo poetico pascoliano. 
lottano in mezzo alle rosate schiume

del lago, e il molle becco è la lor lancia,

e non ferisce sul brocchier di piume.

Guarda le innocue gralle irrequiete

[...]

Ei piange e vede la mia mano ch'apre

rosea, di monte in monte, usci e cancelli;

apre, toccando lieve i chiavistelli,

alle belanti pecore, alle capre;

(Pascoli 2009, 70-2 vv. 49-68, passim)

Nella rivisitazione pascoliana, il duello si trasforma in danza e l'asta del guerriero è divenuta «il molle becco»: alla base di questo particolare è il racconto di Ovidio (Met., XIII, vv. 613-614), ma mentre nel testo ovidiano i colpi sono cruenti («duo diversa populi parte feroces | bella gerunt rostrisque et aduncis unguibus iras | exercent»), nella versione pascoliana «il molle becco... non ferisce» e le gralle sono esplicitamente definite «innocue».

Un procedimento analogo si riscontra nel poemetto latino «Laureolus», composto nel 1893 e stampato nell'anno successivo ad Amsterdam insieme con «Phidyle», per partecipare a un concorso in lingua latina, nel quale «Phidyle» riportò la medaglia d'oro e «Laureolus» il terzo posto. Il poemetto narra la vicenda di due anziani sposi che, tornando la sera alla loro capanna nel bosco, trovano sulla porta un uomo che chiede ospitalità: i due vecchi lo scambiano per il dio Virbio, mentre si tratta di un bandito in fuga, le cui parole sono facilmente equivocate dagli anziani. Quando il bandito sente avvicinarsi le guardie che lo stanno inseguendo, lascia agli anziani la refurtiva che portava con sé e fugge; gli anziani, però, persuasi che si sia trattato della visita del dio, non rivelano nulla alle guardie e fanno sacrifici al dio, convinti che il bottino sia in realtà un suo dono. Il poemetto latino ripercorre la vicenda di Filemone e Bauci, narrata da Ovidio nell'ottavo libro delle Metamorfosi: essa è una vicenda di ospitalità, come nel testo pascoliano, ma anche una riflessione sulla visita prodigiosa del divino all'umano: Giove e Mercurio, che si recano alla povera dimora di Filemone e Bauci, rappresentano un dono, una rivelazione misteriosa e causano la metamorfosi dei due anziani in alberi. Tuttavia lo stesso Pascoli, indicando le fonti del poemetto, cita solamente il passo di Ovidio (Met., XV, v. 543), che riguarda un dettaglio della narrazione, cioè lo scambio tra il brigante e Virbio, divinità minore latina, derivata dalla metamorfosi di Ippolito, figlio di Teseo, operata da Diana, che corrisponde ad Artemide, dea protettrice del giovane, a causa della quale egli scatena l'invidia di Afrodite e perde la vita. La dedica del poemetto alla sorella Maria sottolinea la presenza della fonte ovidiana citata dallo stesso poeta: «Mariae Ioannes sorori dulcissimae, 
Laureolus tu casta meo parce Diana», ed enfatizza il ruolo di Diana, nell'episodio, mentre resta del tutto in ombra la tessitura centrale del testo, ovvero la coppia esemplare Filemone-Bauci, rispecchiata negli anziani coniugi. L'attenzione del poeta si sposta, per così dire, dall'ipertesto dominante a quello secondario, non senza una probabile finalità: la divinità secondaria romana deriva, secondo la notizia ovidiana che Pascoli stesso cita, dalla metamorfosi di Ippolito, vittima innocente delle parole di Fedra e del fraintendimento di Teseo, nell'Ippolito euripideo, così come la salvezza del brigante deriva dal fraintendimento delle sue parole e della sua persona, nel «Laureolus»: in un gioco di specchi rovesciato, Pascoli sembra enunciare un giudizio sulla vicenda tragica, in un'allusione al testo euripideo, del tutto dissimulato, ma presente nella centralità che assume la figura divina nell'errato riconoscimento dei due coniugi. All'amore erotico che domina il testo euripideo si sostituisce la philì pacata e accogliente dei due vecchi, e al contesto tragico quello silvestre, di matrice bucolica, nel quale l'esito funesto si rovescia nella salvezza del brigante.

Sulla scorta del procedimento mitopoietico che costituisce la modalità compositiva delle Metamorfosi ovidiane, Pascoli si muove anche nella formulazione di un vero e proprio mito metamorfico originale, che è rappresentato dal Conviviale «I gemelli». Anche in questo caso, la fonte ovidiana è dissimulata dal poeta; leggiamo infatti nelle «Note alla seconda edizione», del 1905:

L'unico poema nuovo di questa edizione, I gemelli, nasce da un racconto di Pausania... che dice: «C'è un'altra novella su lui (Narcisso) [...], che Narcisso aveva una sorella gemella, come nel rimanente al tutto somigliante di aspetto, così con capellatura uguale, e vestivano vesti simili e andavano a caccia l'un con l'altra. E Narcisso amò la sorella, e come la fanciulla morì, esso andava alla fonte e capiva bensì che era la propria ombra che vedeva, ma pure così capendo, aveva un certo sollievo dell'amor suo, come se non credesse di veder l'ombra sua, ma l'imagine della sorella». Questi due gemelli, non giovani ma fanciulli, io ho cambiati tutti due nel leucoion vernum e nel galanthus nivalis, che si somigliano in verità, ma come un maschietto e una bambina che si somiglino. Sono due fiori del principio di primavera, e della famiglia delle Amarilidee, della quale è pure Narcisso. (Pascoli 2009, 344)

La vicenda è ambientata nel tempo della mitopoiesi, tempo originario in cui nasce l'elaborazione del mito che trasforma in racconto il dolore della vita, ed esprime nel canto la sofferenza:

Ed era il tempo che il nostro dolore cadea qual seme, e ne nasceva un fiore: un fior dal sangue delle nostre vene, 
un fior dal pianto delle nostre pene.

Ed egli fu il leucoio, ella il galantho, il fior campanellino e il bucaneve.

(Pascoli 2009, 275, vv. 89-94)

Con queste concise parole il poeta narra la metamorfosi dei due gemelli in fiori teneri, poco appariscenti e di breve durata, che fioriscono tra febbraio e marzo, nel breve arco cronologico del disgelo, sullo sfondo temporale che è definito in apertura del poema e ripreso nella chiusa:

Spuntava il croco, il morto per amore bel giovinetto. E non fu lor compagno E non l'AI AI videro del giacinto dal vento ucciso. Non fioriva ancora. Erano soli soli; ché la neve era sui monti, era a bacìo, tuttora. E qualche alato, ch'ebbe vita umana già, come loro, già piangea, ma seco, sommessamente: o dentro sé pensava quel pianto amaro ch'è poi dolce canto. I due puri gemelli esili fiori, fu breve la lor vita anche di fiori.

Amor fu quello prima dell'amore.

Non, forse, amore, ma dolor, sì, era.

Sparvero prima della primavera.

(Pascoli 2009, 276, vv. 98-112)

Il racconto che costituisce l'ispirazione centrale, la narrazione ovidiana del mito di Narciso, nel libro terzo delle Metamorfosi, è, per così dire, messo in ombra e ad esso il poeta preferisce la versione di Pausania, in un poema che celebra l'amore fraterno, spezzato dalla morte, con toni che evocano la sensibilità infantile; a differenza del mito ovidiano, in cui l'amore di Narciso per se stesso è una punizione inflittagli da Nemesi per punire il suo rifiuto di Eco, il mito narrato da Pausania consente a Pascoli una ricodificazione che si fa mitopoiesi, anche alla luce della contestualizzazione atemporale che costituisce la cornice del poema; in questo modo la vicenda di un amore solitario ed egocentrico diviene la celebrazione dell'amore fraterno: un mito pienamente legato al mondo pascoliano.

5 Nell'evocazione degli altri fiori, Pascoli fa riferimento ad altri luoghi ovidiani: per il croco, si veda Met., 4, v. 283; per il giacinto Met., 10, vv. 162-219; analogamente, l'allusione all'«alato| ch'ebbe vita umana» rimanda al mito di Procne e Filomela, in Met. 6, vv. 412 e ss., già presente, come si è visto, nel «Poeta degli Iloti». 
La memoria del mito, la rilettura e riformulazione di esso, in Gabriele d'Annunzio è elemento dominante nella poesia delle Laudi, e in particolare di Alcyone, opera nella quale l'ispirazione metamorfica di matrice ovidiana emerge in diverse modalità, fino a culminare nelle figure di invenzione dannunziana, di cui si ha un esempio straordinario in Undulna. Tuttavia, anche in d'Annunzio la presenza delle fonti ovidiane, di cui in questa sede si citeranno solo spunti esemplari, è finalizzata alla creazione di nuovi significati e al tratteggio di una propria identità poetica.

Mito metamorfico per eccellenza, di ispirazione ovidiana, è quello di Glauco, cui d'Annunzio si rivolge attraverso il filtro dantesco, contaminando la narrazione delle Metamorfosi con l'exemplum del «trasumanar»; nel racconto ovidiano Glauco, innamorato della giovane Scilla che lo rifiuta inorridita dal suo aspetto, rievoca tutta la sua vicenda di trasformazione per rendere ragione di quell'aspetto e trovarne giustificazione; il momento della metamorfosi, nonostante sia culminante, è narrato sinteticamente, ed enuncia solo marginalmente due temi che diventeranno fondamentali in d'Annunzio: l'allontanamento dalla terra («'Repetenda' que 'numquam | terra, vale!'», Ov., Met., XIII, vv. 947-948). e la memoria, peraltro circoscritta, della trasformazione («Hactenus acta tibi possum memoranda referre, | hactenus haec memini», Ov., Met., XIII, vv. 955-956).

Nella rivisitazione dannunziana, nel Ditirambo II, la memoria della metamorfosi diviene il centro della rievocazione e offre lo spunto per amplificare l'elemento acquoreo, cui è legata un'intensa suggestione poetica:

Memore sono della metamorfosi.

L'anima si fa pelago

nel rimembrare, s'inazzurra ed èstua,

e le foci vi sboccano

dei mille fiumi che mi confluirono

sul capo: nel rigùrgito

immenso novamente par dissolversi

quest'ossea compagine.

O Iddii profondi, richiamate l'esule

però ch'ei sia miserrimo

nella sua carne d'acro sangue irrigua,

lasso ne' suoi piè debili

che per lotosi tramiti s'attardano,

dopo ch'ei fu l'indomita

forza del flutto convertita in muscoli

tòrtili per attorcere,

dopo che le correnti dell'Oceano

gli furon giogo a tessere 
le divine di sé vicissitudini

come su trama vitrea.

O Iddii profondi, richiamate l'esule

triste, purificatelo

sotto i fiumi lustrali ìnferi e sùperi,

la deità rendetegli!

(Alcione, Dit. II, vv. 9-32)

«Memore» della trasformazione, che viene amplificata nei minimi dettagli esaltati dalla percezione del protagonista, Glauco, non più uomo e non ancora dio, è condannato alla condizione dell'esilio e «immemore» della dimensione umana e terrena, in un'ossessiva rievocazione dell'ambiente acquoreo nel quale è stato immerso e al quale aspirerebbe a tornare, accolto appunto come dio:

«Terra, vale!» Precipite

caddi nel gorgo, mi sommersi, l'infima

toccai valle oceanica, uomo non più, non anco dio, ma immemore

della terra e degli uomini.

(Alcione, Dit. II, vv. 104-108)

L'addio alla terra, tassello esatto del testo ovidiano, diviene il suggello di una sorta di maledizione: al contrario del personaggio antico, per il quale la metamorfosi era semplicemente la condanna al rifiuto della giovane amata, in d'Annunzio essa diviene exemplum dell'estraneità di Glauco all'ambiente nel quale è costretto a ritornare:

O Iddii profondi, richiamate l'esule,

la deità rendetegli!

Io fui Glauco, fui Glauco, quel d'Antèdone.

La terra m'è supplizio.

Ecco, tutta la luce è nel Mare Infero,

e per ovunque è tenebra.

O nunzia di prodigi Alba oceanica!

Nel gorgo mi precipito.

(Alcione, Dit. II, vv. 145-152)

Nel creare il nuovo Glauco, d'Annunzio concentra l'attenzione sull'esperienza del «trasumanar», attraverso il filtro della memoria dantesca, ma aspira proprio a «significar per verba» quell'esperienza che Dante dichiara impossibile, in una sorta di emulazione competitiva:

Nel suo aspetto tal dentro mi fei qual si fe' Glauco nel gustar dell'erba 
che 'l fe' consorte in mar de li altri dei.

Trasumanar significar per verba non si poria.

(Dante, Par., I, vv. 67-70)

Nella figura di Glauco, come è ridisegnata da d'Annunzio, il poeta, che nel narratore-protagonista si identifica, vive l'esperienza di un «trasumanar» e sembra condividere la condizione di esule in un mondo divenuto estraneo, troppo circoscritto per l'aspirazione alla divinizzazione panteistica che anima l'esperienza poetica. Tale processo di identificazione è confermato dalla lirica che segue immediatamente il Ditirambo II, «L'oleandro», nella quale la metamorfosi costituisce il tema centrale, e il poeta, immedesimato in Glauco, interloquisce con figure femminili oggetto anch'esse di metamorfosi: Erigone, che diviene stella; Aretusa, trasformata in fonte, che a sua volta narra la metamorfosi di Dafne; Berenice, il cui ricciolo diviene costellazione, che narra la trasformazione di Cyane.

La presenza di Dante opera in un'altra rilettura di un mito ovidiano, quello di Icaro, rievocata nel Ditirambo IV: in esso il racconto delle Metamorfosi, nell'ottavo libro, è amplificato attraverso la rievocazione di Icaro stesso, io narrante, che dunque riveste lo stesso ruolo dell'Ulisse dantesco nel XXVI canto dell'Inferno, a differenza della narrazione ovidiana nel quale il giovane non ha voce, poiché spetta al padre dapprima impartire i consigli per il volo, quindi richiamare disperato il figlio. Se in Ovidio la voce del padre rappresenta l'insegnamento prudente e il dolore della perdita, nella riscrittura dannunziana la voce di Icaro trasforma il volo nell'impresa estrema, in cui il giovane dà prova del suo desiderio di trasgressione, incurante del pericolo, teso a cogliere l'ebbrezza del superamento della gravità e del limite:

Icaro disse: «Era stellato il cielo, era pacato il mare, nella vigilia mia meravigliosa.

La roggia stella ascosa

nel mio cor vigile era la più grande.

Le cose miserande

eran lungi da me come da un dio

beverato di nèttare novello.

Parea dal corpo snello

dileguarmisi il triste peso come

dal cielo eòo si dileguava l'ombra,

e nella carne sgombra

un aereo sangue irradiarsi.

(Alcione, Dit. IV, vv. 447-459) 
Le spie della corrispondenza testuale con il testo dantesco sono evidenti nelle allusioni letterali (la «vigilia» dei sensi) e nello sfondo stellato del cielo che designano l'apparire dell'«altro polo», nel racconto di Ulisse:

Tutte le stelle già de l'altro polo vedea la notte, e 'l nostro tanto basso, che non surgëa fuor del marin suolo. (Dante, Inf., XXVI, vv. 127-129)

Per estensione, il volo di Icaro diviene «il folle volo» di Ulisse, e il distaccarsi da terra non è più un tentativo di salvezza, ma esperienza fine a se stessa del giovane che sfida la misera realtà terrestre, per farsi quasi divino; e ancora: il «folle volo», nel quale il giovane è misura dell'alba e del tramonto, diviene il volo della sorte, nel quale non la salvezza, ma la prova eroica è il vero fine, senza alcun pensiero del pericolo:

Allor tutte le fiamme

del giorno dal mio cor parvero nate,

per sempre tramontate

dietro di me le stelle della notte,

l'ali della mia sorte

già nel periglio ${ }^{6}$ glorioso aperte.

(Alcione, Dit. IV, vv. 467-472)

Nella silenziosa competizione con Dedalo, Icaro rappresenta il rifiuto della medietà, l'affermazione del gesto sublime:

Il mirabile artiere ebbi in dispregio

silenziosamente. «Al primo volo

io con te lotterò, per superarti.

Fin dal battito primo, io sarò l'emulo

tuo, la mia forza intenderò per vincerti.

E la mia via sarà dovunque, ad imo,

a sommo, in acqua, in fuoco, in gorgo, in nuvola,

sarà dovunque e non nel medio limite,

non nel tuo solco, s'io pur debba perdermi»

risposegli il mio cor silenzioso.

(Alcione, Dit. IV, vv. 504-513)

6 Il «glorioso periglio» richiama a sua volta, i «perigli» che Ulisse rievoca nell'apostrofe ai compagni, in Inf., XXVI, v. 113. 
E nella chiusa, la voce del padre che ritorna dal testo ovidiano, ${ }^{7}$ è un'eco lontana, come è negata la virtù cui Dedalo faceva appello: la virtù per Icaro non si identifica, classicamente, con il senso del limite e della medietà, ${ }^{8} \mathrm{ma}$, in uno slancio sublime, con l'inabissarsi al culmine dell'impresa memorabile:

Gridava «Icaro! Icaro»

il mio padre lontano. «Icaro! Icaro»

$\mathrm{Nel}$ vento e nella romba or sì or no

mi giungeva il suo grido, or sì or no

il mio nome nomato dal timore

giungeva alla mia gioia impetuosa.

«Icaro!» E fu più fievole il richiamo.

«Icaro!» E fu l'estrema volta. Solo

fui, solo e alato nell'immensità.

(Alcione, 1995, vv. 543-551)

Icaro, Icaro, anch'io nel profondo

Mare precipiti, anch'io v'inabissi

la mia virtù, ma in eterno in eterno

il nome mio resti al Mare Profondo!

(Alcione, 1995, vv. 646-650)

E come su Ulisse e sui suoi compagni il mare si richiude, ${ }^{9}$ così esso accoglie il giovane audace, ulteriore controfigura del poeta, che legge nella sua impresa, sulla scorta dell'audacia ulissiaca, l'aspirazione al sublime, al gesto sovrumano, che supera la dimensione terrena, analogamente a quanto si realizza nella riscrittura del mito di Glauco.

La metamorfosi come evoluzione totalizzante, che investe le creature alcionie, si esplica in piena evidenza nella dimensione panica, che vede l'immedesimazione dell'umano con il naturale, di cui sono esemplari le celeberrime trasformazioni che investono Ermione nella «Pioggia nel pineto», o il poeta stesso in «Meriggio» e riproducono la modalità ovidiana della trasformazione da essere vivente a cosa o elemento naturale. Tuttavia, nella rimodulazione costante della forma narrativa metamorfica, esiti più interessanti e originali di Alcyone possono essere considerati le metamorfosi rovesciate, di cui sono

7 Met., VIII, vv. 231-232: «At pater infelix, nec iam pater, 'Icare' dixit,| 'Icare' dixit 'ubi es? qua te regione requiram?'».

8 D’Annunzio 1995, 307, vv. 496-499: «Giova nel medio limite volare: | ché, se tu voli basso, l'acqua aggreva! le penne, se alto voli, te le incende | il fuoco. Tieni sempre il giusto mezzo.| Abbimi duce, séguita il mio solco. | Deh, figliol mio, non esser tropp'oso. | Io ti segno la via. Sii buon seguace».

9 Dante, Inferno, 26, v. 142: «infin che 'l mar fu sovra noi richiuso». 
esemplari le figure femminili di Versilia e di Undulna.

In «Versilia» è infatti la ninfa locale, Versilia appunto, che si cela sotto la scorza del pino accanto al quale è seduto il poeta, a farsi donna per gustare la pesca succosa che egli sbuccia. La metamorfosi è svolta in toni intensi, che, pur trovando riscontro nelle composizioni appena citate, rappresenta, proprio in questo singolare trapassare da forma divina a forma umana, una singolare innovazione; nonostante ciò, la protagonista ricorda i tratti leggiadri di Aretusa, «furace | fauna dei pomarii» («L'Ippocampo», vv. 40-41), «che rapisce la frutta» («L'onda», v. 83) e la figura femminile dell' «Acerba»: «Ti do due labbra fresche per un pugno | di verdi fave» (vv. 7-8) (Alcione, 1995, 196 e 206).

Ancora più suggestiva è la metamorfosi di Undulna, l'onda che traccia le sue danze sulla spiaggia, che diviene divinità marina, specchio della bellezza settembrina dell'estate, sulla quale si chiude il diario alcionio, come preannuncia il poeta nella lirica che precede il testo eponimo:

Estate, bella quando primamente

nella tua bocca il mite oro portavi

come l'Arno i silenzii soavi

porta seco alla foce sua silente!

Ma più bella oggi mentre sei morente

e abbandonata ne' tuoi cieli blavi, che col cùbito languido t'aggravi su la nuvola incesa all'occidente.

T’arda Ermione sul tuo letto roggio gli àcini d'ambra dove si sublima il pianto delle tue pinete australi.

Io della tua bellezza ultima foggio una divinità che su la cima del cuor mi danza: Undulna dai piè d'ali. (Alcione, 1995, 321)

La lirica rappresenta lo sviluppo di un nucleo tematico conservato nel ms. 424, («Undulna - Legge i segni dell'onda come musica scritta»), e nel ms. 425 («Undulna - la dea che presiede alle curve delle onde su la sabbia - che guida le linee segnate dall'onda su la sabbia della riva») e costituisce il più compiuto esempio di mitopoiesi nella trama di Alcyone, secondo quanto il poeta stesso riconobbe, definendo Undulna «La mia vera creatura alcionia».

Divinità nata dalla trasformazione dell'onda, reca i segni del mutamento in una configurazione in cui perdura l'elemento acquoreo e domina la leggerezza danzante dei segni tracciati sulla sabbia: 
Ai piedi ho quattro ali d'alcèdine, ne ho due per mallèolo, azzurre e verdi, che per la salsèdine curvi sanno errori dedurre.

Pellùcide son le mie gambe come la medusa errabonda, che il puro pancrazio e la crambe difforme sorvolano e l'onda.

Io l'onda in misura conduco perché su la riva si spanda con l'alga con l'ulva e col fuco che fànnole amara ghirlanda.

Io règolo il segno lucente che lascian le spume degli orli: l'antico il men novo e il recente io so con bell'arte comporli. (Alcione, 1995, 323, vv. 1-16)

Alla sua metamorfosi si intreccia quella del mese ormai incalzante, Settembre, che assume le sembianze di un flautista, vagante tra i pometi:

Il molle Settembre, il tibìcine dei pomarii, che ha violetti gli occhi come il fiore del glìcine tra i riccioli suoi giovinetti,

fa tanta chiarìa con due ossi di gru modulando un partènio mentre sotto l'ombra dei rossi corbézzoli indulge al suo genio. (Alcione, 1995, 323, vv. 63-72)

A poco a poco, nell'avanzare della pacata stagione settembrina, nella voce di Undulna sembra risuonare quella del poeta, che contempla attraverso lo sguardo della ninfa lo scenario ormai immobile:

Silenzio di morte divina per le chiarità solitarie! Trapassa l'Estate, supina nel grande oro della cesarie.

Mi soffermo, intenta al trapasso. Onda non si leva. Lalbèdine 
è immota. Odo fremere in basso, a' miei piedi, l'ali d'alcèdine.

(Alcione, 1995, 329, vv. 101-103)

\section{$[\ldots]$}

Azzurre son l'ombre sul mare

come sparti fiori d'acònito.

Il lor tremolìo fa tremare

l'Infinito al mio sguardo attonito.

(Alcione, 1995, 330, vv. 125-129)

Nella figura di Undulna, ultima divinità alcionia, si confondono lo sguardo e la voce di d'Annunzio, che in questa creazione è ancora una volta allievo di Ovidio nell'utilizzarne l'ideazione poetica per un'invenzione del tutto originale.

Ovidio, che, come si diceva, sembra a prima vista estraneo al mondo poetico di Pascoli, diviene in realtà, per questo poeta, una suggestione ricca di derivazioni e deviazioni, dalle quali emerge il tracciato di un'eredità vivificata secondo la rilettura profondamente creativa che anima le pagine pascoliane, mentre sullo sfondo si può intravvedere l'immagine di quell'antico esule, di cui risuona la frustrazione e la malinconia. Solamente di passaggio vale la pena di osservare che nell'antologia dedicata alla poesia lirica, Lyra, Pascoli ricostruisce le figure dei poeti antichi, soprattutto Catullo e Orazio, creandone veri e propri profili biografici animati da sentimenti e valori in gran parte costruiti dal poeta sulla scorta della sua sensibilità e del procedimento di vivificazione dell'antico che egli teorizza in numerosi scritti critici: ${ }^{10}$ analogo procedimento interessa la figura di Omero, nell'antologia della poesia epica Epos. La figura di Ovidio non ha trovato, da parte di Pascoli, rielaborazione, perché, come si è visto, l'antologia dedicata all'elegia è rimasta allo stadio progettuale; tuttavia un'immagine sembra richiamare la figura umana di quel poeta, nella sua vecchiaia infelice, condannato all'esilio: il protagonista de «Il cieco di Chio» non rappresenta solo l'anziano Omero, ma forse anche Ovidio, relegato lontano da Roma, "cui poter falla e desiderio avanza» (Pascoli 2009, 28, v. 9), nell'altalenante e lacerante condizione dell'aspirazione al canto e della frustrazione.

In d'Annunzio, al contrario, la traccia del poeta antico, tanto più evidente sulla superficie, tende a svanire, ma nutre modalità di lettura contrapposte: nei due «Ditirambi» contribuisce a creare l'immagine dell'eroe che possiede solo memoria o solo rimpianto, come il poeta

10 Per la disamina su questo procedimento si rimanda agli studi fondamentali di Ferratini (1990) e Baroncini (2005). 
che se ne fa cantore; in «Undulna» ispira l'esperimento della mitopoiesi contemporanea, in parallelo a «I gemelli» pascoliani: là il mito dell'affetto familiare, qui la grazia della figura femminile, che si fa onda.

Proprio in questa diversità di rilettura consiste forse l'interpretazione più vera dell'opera e della figura di Ovidio, la cui ricchezza è fonte di ricodificazioni mai univoche, come sembra acutamente osservare una disincantata scrittrice australiana contemporanea, incaricata dal suo editore di tradurre piuttosto liberamente Ovidio:

Non sono sicura di aver già accennato alla scadenza di Ovidio: ventiquattro racconti in versi in centouno giorni. Un sacco di soldi a lavorare così, ci puoi scommettere. Una specie di traduzione, ma solo come punto di partenza: sto facendo dei cambiamenti nelle storie. E credo che Ovidio, fra tutti, sarebbe l'ultimo a dispiacersene. (Alison 2018, 265)

Le pagine che precedono riproducono l'intervento tenuto nel corso della Settimana della Classe di Lettere presso l'Ateneo di Brescia, Ovidio: temi e suggestioni, nell'ottobre 2018.

\section{Bibliografia}

Alison, Jane (2018). Meglio sole che nuvole. Leggere Ovidio a Miami. Milano: NN. Baroncini, Daniela (a cura di) (2005). Giovanni Pascoli, Letture dell'antico. Roma: Carocci.

Belponer, Maria; Gibellini, Pietro (a cura di) (1995). D’Annunzio, Gabriele: Alcione. Milano: Garzanti.

Belponer, Maria (a cura di) (2009). Pascoli, Giovanni: Poemi conviviali. Milano: BUR.

Calvino, Italo (1979). «Gli indistinti confini» . Ovidio: Metamorfosi. Torino: Einaudi, VII-XVI.

Ferratini, Paolo (1990). I fiori sulle rovine. Pascoli e l'arte del commento. Bologna: il Mulino.

Magugliani, Lodovico (a cura di) (1979). Esiodo: Le opere e i giorni. Milano: BUR. 


\title{
Contro i nuovi Barbari: d'Annunzio, Spengler, l'ombra di Cesare
}

Alfredo Sgroi

Università degli Studi di Catania, Italia

\begin{abstract}
In the European culture between the nineteenth and twentieth centuries there is a denunciation of the decline of Western civilization, with the expectation of a ransom. The new Caesarism is the dream of many intellectuals. D'Annunzio and Spengler are protagonists in this cultural phase. The Italian writer anticipates the German philosopher. In fact, works like Le vergini delle rocce confirm the many convergences with Der untergand der Abendland, published in 1918. In this essay we analyse the analogies between the Weltanschauung of the two authors.
\end{abstract}

Keywords Sunset. Degeneration. Redemption. Aristocracy. Caesarism.

$\begin{array}{ll}\text { Peer review } & \\ \text { Submitted } & 2019-03-11 \\ \text { Accepted } & 2019-05-23 \\ \text { Published } & 2019-10-15 \\ \text { Open access } & \end{array}$

(C) 2019 | ()( Creative Commons Attribution 4.0 International Public License

Citation Sgroi, Alfredo (2019). "Contro i nuovi Barbari: d’Annunzio, Spengler, l'ombra di Cesare". Archivio d'Annunzio, 6, 85-100. 
Si intrecciano nella cultura europea a cavallo tra Ottocento e Novecento l'ossessiva denuncia dell'irreversibile declino della civiltà occidentale, da un lato, l'ansiosa attesa di un riscatto sotto il segno di un nuovo cesarismo, dall'altro. Queste due pulsioni profonde, e sovente operanti in maniera speculare, non potevano non segnare il percorso artistico e intellettuale di uno scrittore acutamente sensibile e aperto alle più aggiornate suggestioni quale Gabriele d'Annunzio. Anzi, si può dire che egli sia stato solerte nel captare quanto covava nel seno della cultura filosofica e politica di fine secolo. E capace di proiettare verso il futuro il groviglio di attese e inquietudini che sfoceranno, negli anni immediatamente successivi al primo conflitto mondiale, nel grande affresco crepuscolare tracciato da Oswald Spengler. D'Annunzio, infatti, è stato al contempo cantore accorato della decadenza e profeta di un riscatto che passa per un rovesciamento della società borghese-democratica. E in questo senso maître à penser da cui non poteva prescindere chi, come Spengler, all'indomani della fine della Grande Guerra è costretto a fare i conti con un'endemica condizione di conflittualità sociale. E che in un radicale «ritorno all'ordine» individua l'inevitabile sbocco per porre fine ai tumulti che turbano tante coscienze. Di più: la concezione del «tramonto dell'occidente», infetto dalla corruzione innescata dall'irruzione della società di massa, si salda con l'indicazione di un'unica via d'uscita: un ritorno all'indietro con il ripristino dei valori aristocratici.

Disgusto e stanchezza alimentano prepotentemente sia in d'Annunzio che in Spengler l'attesa messianica dell'uomo d'ordine. Un nuovo Cesare, appunto. Antiborghese, antidemocratico, antisocialista. Pacificatore, custode e restauratore delle virtù profonde della stirpe. Populista eppure aristocratico.

I primi sintomi, netti, di questo clima ideologico si manifestano precocemente anche in Italia, anzitutto per effetto della deludente conclusione del processo risorgimentale, con la cocente frustrazione delle pulsioni imperiali. Peraltro nello stesso lasso di tempo in cui il cesarismo francese, incarnato da Napoleone III, sta per franare sotto i colpi della barbarie prussiana. Nel settembre del 1868, infatti, Giosuè Carducci compone un dittico (due sonetti): Il cesarism (Giardina-Vauchez 2000, 171-2; Carducci 1963, 45-9), a ridosso della pubblicazione della Histoire de Jules César firmata nel 1865 proprio da Napoleone III. Opera, questa, in cui ricompaiono le medesime celebrazioni delle dittature provvidenziali, come quella di Cesare, alle quali spetta il compito di soffocare quelle situazioni di disordine che, periodicamente, si ripresentano nella storia. Dopo le guerre civili, infatti, nella Roma antica arriva Cesare. Dopo i torbidi della rivoluzione del 1789, arriva Napoleone I. A chiudere la nefasta fase innescata dalle rivolte del 1848, arriva Napoleone III. La storia si ripete e, sotto il segno di un progetto provvidenziale, che evita alle nazioni di sprofondare nel caos. 
Di cesarismo ragionava in quegli anni anche Mazzini, sempre in quell'ottica provvidenziale ribadita, ma con un sottofondo di sarcastica ironia, da Carducci: "Giove ha Cesare in cura», proclamava infatti lo scettico poeta tanto venerato dal giovane d'Annunzio. ${ }^{1}$ Resta il fatto che i sussulti che avevano scosso l'intera Europa a partire dalla metà dell'Ottocento avevano alimentato tra gli intellettuali (e non solo) un diffuso desiderio di un rapido «ritorno all'ordine». Lo conferma la copiosa produzione letterario-filosofica che spazia da Carlyle a Nietzsche, in cui circola la nostalgia per la dorata società aristocratica spazzata via dalle rivoluzioni borghesi; e l'inquietudine per l'irrompere delle masse, con le loro istanze egualitarie. C'è voglia di ordine, dunque, e di gerarchia: di fuga da una realtà caotica, cupa, nemica della bellezza. Come contrappeso alla dilagante 'degenerazione' si vagheggia perciò, sulla scia di Nietzsche, il ripristino dei valori aristocratici. E con l'acuirsi della crisi, nella fase declinante della belle époque, e la conseguente deflagrazione bellica del 1914, ancor più cresce il desiderio, o la convinzione, che un nuovo cesarismo sia alle porte.

In questo contesto Nietzsche diviene il punto di riferimento ineludibile. Giova rammentare, tra le invettive scagliate dal filosofo contro la degenerazione della civiltà occidentale, una pagina del Così parlò Zarathustra (Nietzsche [1883-85] 2011, 283-7). In essa sono protagonisti due re, che si imbattono nel profeta-eremita, e con lui intrecciano un rapido scambio di battute sull'eclissi della funzione regale. Ma prima c'è un martellante atto d'accusa contro una società in cui l'avvento della morale 'plebea' ha a tal punto disgustato chi, come i due re, si sente a essa tanto estraneo da non considerare praticabile alcun'altra alternativa alla fuga precipitosa. Questi sovrani che cercano nel regno di Zarathustra la salvezza, sono l'emblema di una civiltà aristocratica spazzata via dall'«intruglio plebeo»; di un tipo superiore di umanità 'ascendente' che disprezza il nauseante crogiolo di uomini senza identità, immersi appunto in un «intruglio» nel quale «tutto è mescolato alla rinfusa»: in quelle metropoli moderne che, come scriverà Spengler, sono i luoghi in cui l'infezione della decadenza è più aspra.

La «puzza dei mercanti», il marciume della cosiddetta buona società, rende irrespirabile l'aria per i due sovrani, che a Zarathustra annunciano con amarezza che «è meglio vivere in mezzo a eremiti e caprai»; e che è preferibile il tipo rozzo e testardo dell'antico contadino alla marea borghese (la «canaglia affarista»); che, scontata la pena del tramonto dell'idea stessa di regalità («che mai contano ancora i re»), connessa alla più nefasta degenerazione della civiltà («Rovina! Rovina! Mai cadde così in basso il mondo! Roma si degradò a puttana di

1 Il termine viene coniato in Francia intorno al 1850 da François Auguste Romien (cf. Mangoni 1979, 17-52). 
bordello, Bestia divenne il Cesare romano»), (Colli, Montanari 2011, 286) è arrivato il momento dell'esilio. Tocca a Nietzsche-Zarathustra ribattere che è giunto il tempo dell'attesa di una nuova epoca di là da venire, quando risorgeranno i valori dell'aristocrazia e tornerà il tempo propizio per i re.

D'Annunzio si attesta sulle medesime posizioni ideologiche-estetiche. Esse, se da una parte recepiscono le suggestioni che, come detto, sedimentano in tanta cultura ottocentesca, dall'altro prefigurano una nuova stagione: quella che sarà consacrata dalla riflessione di Oswald Spengler e sfocerà nell'apoteosi cesarista celebrata dal fascismo. Cesarismo che, a ben considerare, è in fondo una variante dell'utopia neo-monarchica sostenuta da d'Annunzio.

Il sogno aristocratico è celebrato già negli anni trascorsi a Roma, consumati tra letti e salotti; tra cronache mondane e sciali scandalosi. Prosegue a Napoli, là dove assume una declinazione più politica, e deflagra poi nelle pagine del Convito e del romanzo del 1895: Le vergini delle rocce (Andreoli, Lorenzini 1989, 2: 1-193), intrecciato alla denuncia della condizione patologica della modernità, ${ }^{2}$ ossessivamente presente negli articoli di questi medesimi anni, in cui si profila una visione morfologica della storia (alla Spengler). Qualche esempio: in un articolo apparso su Il Mattino tra l'11 e il 12 luglio del 1892 si legge: «le creature veramente ideali non nascono che nelle epoche primitive. Bisogna risalire all'origine dei popoli, alle albe dell'infanzia umana, per trovare gli eroi: che i popoli creano dal loro cuore e nutrono dei loro sogni» (Andreoli 1996, 32).

Albe e tramonti scandiscono il perenne fluire circolare dei corsi e ricorsi storici (Conte 2009), seguendo la medesima dialettica su cui si fonda la concezione spengleriana, nella quale si incrociano le stesse suggestioni nietzschiane assorbite dallo scrittore italiano. E non si tratta soltanto di un generico eterno ritorno del medesimo: perché anche sul piano etico-estetico tutto si ripete: «nell'arte del passato c'è una parte eterna che rivive in ogni età» (Andreoli 1996, 45).

Nelle Note su l'arte del 31 agosto-1 settembre del 1892 d'Annunzio teorizza il nesso indissolubile tra decadenza e «estrema civiltà». In termini spengleriani, tra degenerazione e «civilizzazione». Zivilisation in cui, noterà il tedesco, si compie il percorso di sradicamento spaesante dell'individuo rispetto alla realtà in cui vive: una realtà dominata dallo spettro angosciante della metropoli, considerata co-

2 A Ugo Ojetti nel 1895, d’Annunzio confida: «la malattia [...] concorre ad allargare i il campo della conoscenza. Lo studio dei degenerati, degli idioti, dei pazzi è per la psicologia contemporanea uno dei più efficaci modelli di speculazione, perché la malattia aiuta l'opera dell'analisi decomponendo lo spirito. Essendo un disordine patologico l'esagerazione d'un fenomeno naturale, la malattia fa l'officio di uno degli strumenti che servono ad isolare e ad ingrandire la parte osservata. In fatti, le conquiste più notevoli della psicologia contemporanea sono dovute a psichiatri» (Andreoli 1996, 1388). 
me un grande gorgo che ingoia il soggetto, lo destabilizza, ne mina dalle fondamenta l'equilibrio nervoso. D’Annunzio annuncia:

Questa inquieta aspirazione ad escir fuori della realtà mediocre, questo desiderio vago di trascendere l'angustia della vita comune, questa smania quasi incosciente di vivere una vita più appassionata e più complessa sono proprio delle epoche di decadenza, delle epoche di estrema civiltà e di estrema debolezza. (73)

La condizione crepuscolare è dunque quella in cui ormai è sprofondata la civiltà occidentale. In essa, nella visione dannunziana, si inseguono in una tragica sfilata mortuaria, il «crepuscolo dei re e dei principi» (81), ${ }^{3} \mathrm{il}$ «crepuscolo dei popoli» (Nordau [1895] 2009, 11-22), quello degli «dei»; è «un'epoca di vita discendente», ma in cui pure è in incubazione la voglia di riscatto, l'ideale «di vita ascendente» (Andreoli 1996, 232; corsivi nell'originale). Un tramonto, in termini spengleriani, a cui si contrappone l'attesa di una nuova alba.

Contro la patologica cupio dissolvi, celebrata perfino nelle superiori manifestazioni artistiche, ${ }^{4}$ occorre ingaggiare una guerra implacabile e senza quartiere; una lotta per riconsacrare i diritti della forza, dell'affermazione violenta e vitalistica. In definitiva, una naturale gerarchia, al sommo della quale si deve ricollocare una nuova aristocrazia: «L'aristocrazia nuova si formerà dunque ricollocando nel suo posto d'onore il sentimento della potenza levandosi sopra il bene e sopra il male» (92; corsivi nell'originale). ${ }^{5}$

La lezione di Nietzsche, trasparente in questo passo, spalanca quindi una prospettiva palingenetica di riscatto dalla decadenza, di superamento della patologica Zivilisation, possibile assecondando la necessaria curvatura della storia; espellendo i languori pessimistici e evangelici coltivati da uno Schopenhauer o da un campione della 'degenerazione' come Tolstoj (112); ricomponendo sotto l'egida dell'arte e della bellezza il legame con quella natura da cui l'uomo ha colpevolmente divorziato. Insomma, ritornando alla condizione dionisiaca, al di qua delle «malattie dello spirito» che corrodono l'occidente. Poiché l'aristocrazia - sosterrà Spengler - è l'espressione più vitale di una Kultur all'apice della sua ascesa.

D’Annunzio travasa quanto elabora nei suoi articoli-saggi nell'opera in prosa e in versi, prima ancora che nella sua azione politica. Co-

3 Analoghi concetti sono ribaditi nel discorso Agli elettori di Ortona e nel Proemio al Convito (cf. Andreoli 1996, 283-6).

4 «Nulla è oggi studiato meglio che il carattere proteiforme della degenerazione», in una società di «esauriti e spossati» (cf. Andreoli 1996, 245-51).

5 Nel noto articolo del 23 luglio 1893, Il caso Wagner, è evocata la ineluttabile necessità di una nuova aristocrazia, parafrasando il Nietzsche della Genealogia della morale. Non a caso il filosofo è definito «un rivoluzionario aristocratico» (Andreoli 1996, 237) 
sì, a proposito di Tullio Hermil, protagonista dell'Innocente, Ugo Caferio, ispirato dallo scrittore, evoca un'«anarchia aristocratica» che porta a rigettare «quella morale» che ha impresse le stimmate della 'degenerazione' (155). Ancor più questa operazione osmotica è visibile nel ricordato romanzo Le vergini delle rocce. Vero e proprio condensato delle ossessioni politico-estetiche dello scrittore. E autentica summa della concezione neoaristocratica dannunziana, concepita come l'unico antidoto nei confronti della dilagante degenerazione della moderna civiltà (specialmente delle nazioni latine).

A incarnare la dialettica relazione tra decadenza e riscatto aristocratico è il protagonista dell'opera, Claudio Cantelmo Alter ego dell'autore, anzi, suo vero e proprio autoritratto idealizzato, incarnazione delle sue aspirazioni, frutto di un sincretismo ideologico in cui confluiscono diverse componenti, anche contraddittorie.

All'aspra critica del sistema di valori su cui si fonda la moderna società borghese è connesso un velleitario progetto palingenetico: generare il nuovo re di Roma, a cui spetterà il compito di attuare l'avvento della «rinascenza latina» e il ripristino di quei valori aristocratici profondamente corrosi dal tarlo della degenerazione. Un progetto, questo, nel quale utopia e nostalgia si mescolano in una singolare miscela. La medesima a cui attingerà la cosiddetta 'rivoluzione conservatrice'.

Nel Libro primo del romanzo del 1895 è stigmatizzata la civiltà borghese, immiserita dalla grettezza ipocrita che governa una società in cui non c'è più spazio per l'antica grandezza dei valori eroici. Il trionfo di un grigiore mediocre si spande infatti su tutti gli aspetti della vita sociale e intellettuale, sulla scia del «gran dogma dell'89», che postula l'eguaglianza tra gli uomini.

L'invettiva antiborghese, in gran parte il frutto di una rielaborazione dell'articolo La bestia elettiva (86-94), si innesta sullo sfondo della Roma Umbertina. Luogo emblematico in quanto metropoli dai tratti sempre più marcatamente borghesi (e quindi decadenti), nella quale si manifesta il «dissolvimento morale e materiale della giovane nazione italiana». ${ }^{6} \mathrm{E}$ si misura tutta la portata del declino dell'aristocrazia, fisicamente spazzata via dall'irrompere nel tessuto urbano della nuova, rozza, classe sociale in rapida ascesa. Un'aristocrazia al 'tramonto', quindi, condannata all'estinzione dalla dinamica circolare della storia. A essa deve subentrarne una ascendente, a segnare una nuova 'alba'. Perciò Cantelmo vorrebbe rivitalizzare le virtù sopite della sua stirpe, ponendosi agli antipodi rispetto alla squallida realtà in cui vive. Da qui parte la sfida lanciata al presente tarato dalla malattia borghese. A lui d'Annunzio fa dire:

6 Il 10 luglio del 1895 d'Annunzio scrive a Treves: «Penso che la parte politica delle Vergini non potrà non avere qualche effetto sul volgo. Vi sono parole incise con mano assai fiera» (Oliva 1999, 162-4: 164). 
Vivendo in Roma, io era testimonio delle più ignominiose violazioni e dei più osceni connubii che mai abbiano disonorato un luogo sacro. Come nel chiuso d'una foresta infame, i malfattori si adunavano entro la cerchia fatale della città divina [...] Come un rigurgito di cloache l'onda delle basse cupidigie invadeva le piazze e i trivii, sempre più putrida e più gonfia. (Andreoli, Lorenzini 1989, 19)

Situazione, questa, che è in realtà il paradigma di una condizione universale. In questo degradato contesto pontefici e sovrani sono ridotti a scialbi esecutori del «decreto fatto dalla plebe», travolti dalla secolare ascesa della morale della rinuncia e dalla sorda lotta contro tutto ciò che di nobile e bello è stato prodotto dall'antica società aristocratica. Urge perciò la figura carismatica di un re-condottiero in grado di disciplinare il «torbido bollore di passioni servili», di soggiogare la classe borghese e avviare la riscossa. Perciò è necessario un percorso di purificazione. Una catarsi che può essere realizzata soltanto esiliandosi dalla città infetta. Così il personaggio abbandona, scorato ma deciso a non soccombere, la Roma ridotta a covo di una borghesia rapace e di una nobiltà sfatta dalla corruzione. A conferma del ruolo paradigmatico dell'Urbe, metafora della civiltà occidentale giunta al suo tramonto. Eppure luogo fatale in cui la risorta aristocrazia dovrà insediarsi per fare risorgere i fasti imperiali. Nel segno del mito della «terza Roma», che tanta parte avrà poi nell'immaginario collettivo in epoca fascista (cf. Giardina, Vauchez, 2000, 58-116).

Roma, pur profanata dal saccheggio devastante della speculazione edilizia, resta comunque la «Madre» delle nazioni. Pur deturpata dal trionfo del «grigio diluvio democratico», può essere ancora riscattata da un re-eroe e potrà generare un nuovo impero. Tocca agli intellettuali il compito di definire un programma etico-estetico in grado di arginare la decadenza e ripristinare il culto del Bello e della gerarchia. Occorre realizzare un'alleanza tra gli stessi intellettuali e gli aristocratici per generare una nuova stirpe di Eroi-esteti. Il tempo sembra maturo. Serve un luogo propizio, puro, che non può essere lo spazio metropolitano in cui alligna il germe della decadenza. L'unica alternativa possibile è dunque il ritorno alla terra: a quella campagna non contaminata dall'infezione democratico-borghese, nella quale si sono affermate quelle classi sociali (aristocrazia e contadini), che con la stessa madre-terra hanno un saldo legame identitario.

Cantelmo si reca dunque nel suo antico feudo di Rebursa, là dove pensa di potere realizzare il suo progetto di riscatto, lontano dalla città profanata dai nuovi barbari e dagli «schiavi ubriachi», e dai patrizi «spogliati d'autorità in nome dell'eguaglianza. Relitti traditori della loro stirpe, «ignari o immemori delle arti di dominio professate dai loro avi» (Andreoli, Lorenzini 1989, 29). Incapaci di cogliere l'intrinseca fragilità della 'civilizzazione' che annulla le identità per dare vita a una massa indistinta di individui senza qualità: 
Per fortuna lo Stato eretto su le basi del suffragio popolare e dell'uguaglianza, cementato dalla paura, non è soltanto una costruzione ignobile ma è anche precaria. Lo Stato non deve essere se non un istituto perfettamente adatto a favorire la graduale elevazione di una classe privilegiata verso un'ideal forma di esistenza [...] Non vi sarà troppo difficile, in vero, ricondurre il gregge all'obbedienza. Le plebi restano sempre schiave, avendo un nativo bisogno di tendere i polsi ai vincoli. (30-1)

Una visione ottimistica, in fondo. Una vera e propria utopia all'insegna della rivoluzione conservatrice fondata sull'attesa messianica di una nuova stirpe di eroi. Nessun dubbio sembra incrinare questa speranza palingenetica:

Quando tutto sarà profanato, quando tutti gli altari del pensiero e della Bellezza saranno abbattuti, quando tutte le urne delle essenze ideali saranno infrante, quando la vita comune sarà discesa a un tal limite di degradazione che sembri impossibile sorpassarlo, quando nella grande oscurità si sarà spenta pur l'ultima fiaccola fumosa, allora la Folla si arresterà presa da un panico ben più tremendo di quanti mai squassarono la sua anima miserabile; e, mancata a un tratto la frenesia che l'accecava, ella si sentirà perduta nel suo deserto ingombro di rovine, non vedendo innanzi a sé alcuna via [...] Allora scenderà su lei la necessità degli Eroi [...] io penso che questi Eroi debbano sorgere dalla nostra razza. (153)

Ma il passaggio dalla Roma devastata dalla speculazione edilizia verso la campagna incontaminata di Rebursa non sottrae il protagonista dal generale clima di disfacimento della modernità. Si rovescia, al contrario, e smentendo clamorosamente i suoi proclami, in una nuova immersione negli inferi della decadenza. ${ }^{7}$

Il contagio, dunque, è universale. E in questo quadro apocalittico si giustifica anche la crisi che ha investito il ruolo dell'artista, non più divino demiurgo, ma nostalgico depositario di quegli stessi ideali aristocratici spazzati via dal trionfo della civiltà borghese. Né, alla fine, si intravede quel monarca-esteta capace di coniugare i valori eroici con le ragioni dell'arte, e di realizzare una trasvalutazione dei valori che possa invertire il corso declinante della storia.

$\grave{E}$ in questo orizzonte ideologico che si colloca anche Elettra, divisa in due sezioni: da una parte le composizioni dedicate all'esaltazione

7 Si registra qui il medesimo fallimento politico che sarà da lì a poco, nel 1899, al centro del plot della tragedia La Gloria. Si può dire, infatti, che la parabola di Ruggero Flamma, prima acceso rivoluzionario e poi imbelle e corrotto dittatore, ripropone la stessa traiettoria fallimentare. 
degli eroi, con cui il poeta accoglie l'eredità di Carducci e si accredita come nuovo Vate della giovane nazione italiana; dall'altra la serie delle «città del silenzio» (Andreoli, Lorenzini, 1984, 2: 253-410). Nella prima vengono proposte alcune delle più significative, e ridondanti, odi civili composte a cavallo del 1900. Come l'appassionata canzone $A$ Dante (1899), nella quale il poeta fiorentino assurge al ruolo di ispirato profeta dei destini dell'Italia. O, ancora, Al Re giovine (1900), scritta a ridosso del drammatico regicidio di fine secolo, nella quale si invita il nuovo sovrano Vittorio Emanuele III a porsi alla guida del riscatto.

Perno ideale e simbolico resta, nonostante tutto, l'Urbe. Nell'ode $A$ Roma (1900), infatti, si riafferma il destino fatale della città, che diverrà ancora una volta ciò che fu nel glorioso passato imperiale. Perché tutto ritorna. Quasi provvidenzialmente, secondo un disegno che nulla ha di religioso. L'attesa è però frustrata e spinge il poeta, col trascorrere del tempo, a cercare l'urto dell'azione, a attendere, tra ansie e delusioni, il momento propizio per scendere in lizza e divenire egli stesso protagonista attivo della rinascita del mito della romanità:

O Roma, o Roma, in te sola, | nel cerchio delle tue sette cime, | le discordi miriadi umane | troveranno ancora l'ampia e sublime | unità. Darai tu il novo pane | dicendo la nuova parola. (Andreoli, Lorenzini 1984, 283)

D'Annunzio sa che l'arte non basta più: ci vuole uno scatto in avanti. Un'immersione nel sangue e nella carne dei conflitti della storia. E l'occasione arriva finalmente, forse ormai insperata, con lo scoppio della Prima guerra mondiale. Ė il tempo esaltante dei Canti della guerra latina (1914-1918) (Andreoli, Lorenzini 1984, 759-872). ${ }^{8} \mathrm{Ma}$ soprattutto della lotta nei campi di battaglia. Ed è, anche, l'estremo canto del cigno del poeta ormai anche soldato, destinato però malinconicamente alla rapida eclissi, soppiantato dal ben più scaltro Mussolini, e a rimanere prigioniero nella gabbia dorata del Vittoriale. Ma prima il verbo si fa concreta realtà, e incisiva ma velleitaria azione politica, nell'effimera epopea consumata a Fiume tra ritualità e atmosfere riesumate attingendo a piene mani nel ricco serbatoio della tradizione romana (cf. Salaris 2002, 23 e ss.). ${ }^{9}$

A Fiume d'Annunzio può infatti, finalmente, vestire i panni del nuovo duce. Può realizzare per un breve tempo il sogno di grandezza ormai traslato dal regno metafisico dell'arte a quello immanente dell'azione. ${ }^{10}$ Convinto che soltanto in questa maniera, lanciandosi

8 Si legga anche Per la più grande Italia, edita da Treves nel 1905. In particolare la sezione «La legge di Roma» (cf. Andreoli, Lorenzini 1984, 65-109).

9 Una testimonianza diretta si legge in Carli 1920.

10 Cf. De Felice 1978 e De Felice, Gibellini 1987. 
nella lotta, si può davvero invertire il corso declinante della civiltà; perché la guerra ha una sua etica vigorosa, che può svecchiare e rigenerare l'infiacchita civiltà occidentale, rinsaldando la comunità e rivitalizzando il senso di appartenenza alla nazione..$^{11}$ L'astro dannunziano volge però al tramonto proprio quando si compie la metamorfosi da artista a uomo d'azione.

Per una singolare coincidenza, proprio mentre il poeta si cimenta nell'impresa fiumana, irrompe sulla scena della cultura europea lo spengleriano Il tramonto dell'occidente, con effetti dirompenti. ${ }^{12}$ Lo conferma anzitutto, paradossalmente, la stroncatura crociana del 1920 (sulla Critica), ${ }^{13}$ che per contrasto doveva suscitare una reazione di segno diverso tra gli anticrociani (come d'Annunzio), e anche l'interesse mostrato da Mussolini, che ne appoggia personalmente la pronta ricezione in Italia, tramite soprattutto il filtro di Vito Beonio Brocchieri (Conte 1994, 83 e ss.).

Tralasciando la questione del complesso rapporto tra Spengler e il fascismo, in questa sede non si può non segnalare che l'esplodere del cosiddetto fall Spengler, sulla cui scia diventa familiare la celebre distinzione tra Kultur e Zivilisation, non sarà certamente sfuggito a d'Annunzio. Né il silenzio glaciale che l'artista italiano riserva a quello che spregiativamente era considerato un semplice epigono di Nietzsche (da Troeltsch, ad esempio) è sicuro indizio di noncuranza. Possiamo semmai immaginare un d'Annunzio che vede proprio nell'opera spengleriana l'ennesima conferma dell'esattezza della sua impietosa analisi della decadenza occidentale. Come pure della condanna senza appello del modello metropolitano. ${ }^{14}$ Basti qui considerare i punti salienti delle più evidenti convergenze ideologiche, che giustificano ampiamente una lettura speculare dei due autori.

Il senso autentico della storia, per Spengler come per d'Annunzio, è dato dall'eterno ritorno delle medesime "correnti vitali»; da una ciclicità in cui si alternano fasi di espansione e abissi di decadenza. L'epoca attuale, dominata in occidente dalla comparsa di quello che Spengler chiama «uomo faustiano», dalla connessa, graduale, degenerazione suscitata dal ripiegarsi sempre più radicale del soggetto

11 Per uno sguardo di lungo periodo sul tema si veda Gentile 1996. Sulla diffusione del mito della guerra negli ambienti intellettuali internazionali, in particolare tedeschi, fondamentale Losurdo 2001, in particolare alle pagine 3-23.

12 Evola 1991. Recentemente la prima parte è stata ripubblicata con traduzione a cura di Giuseppe Raciti (2017).

13 La recensione si legge ora in Croce 1950, 313-7. Non meno aspra è anche quella riservata al successivo L'uomo e la tecnica, ora in Croce 1939, 294-8.

14 Nella metropoli si celebra anche il trionfo della tecnica che smorza, fino a soffocarli del tutto, quegli istinti predatori propri dell'umanità nel suo pieno vigore (cf. Raciti, a cura di, 2016, 106-10). Su questi temi si veda almeno Cacciatore 2005, in particolare alle pagine 71-6. 
in se stesso, dall'avvento della prospettiva nichilista, ${ }^{15}$ costituisce l'estremo bagliore di una civiltà giunta alla sua fase di «senescenza». Il che non è un'eccezione, perché il ciclo nascita-maturazione-decomposizione è il tratto caratteristico di tutte le civiltà apparse sulla Terra.

Dietro l'angolo, argomenta Spengler, c'è ormai in Occidente un effimero tentativo di rinascita fondato sul mito dell'epifania di quella che fu la Roma dei Cesari. ${ }^{16} \mathrm{E}$ il disseppellimento, altrettanto fugace, dell'etica aristocratica. Perché quella «non classe» che è la borghesia metropolitana ha determinato l'asfissia etica e culturale delle classi autentiche: quella sacerdotale e, soprattutto, quella aristocratica. Quest'ultima esprime per il filosofo il senso della storia nella sua massima espressione:

Così in tutte le civiltà superiori vi è un elemento contadino il quale è razza epperò, in certo modo, natura, e vi è una società che è pretenziosamente «in forma», costituita da un gruppo di classi o di caste, come qualcosa di più artificiale e di più transitorio. Ma la storia di queste classi e di queste caste è storia mondiale nella sua estrema potenza. (Evola 1991, 1138)

Una sorta di peccato originale è dunque per Spengler all'origine della moderna civiltà giunta al suo tramonto (Untergang), e che in sé porta impresse le stimmate funeste della borghesia che l'ha foggiata a sua immagine e somiglianza, imponendo la supremazia della ragione economica; la dittatura del denaro; una Weltanschauung basata sulla categoria esclusiva dell'utile. Privo di identità com'è, il ceto borghese disprezza come retaggio del passato ciò che custodisce nel suo seno i valori tradizionali, suprema espressione di quella raffinata civiltà aristocratica che ha per secoli brillato nel campo dell'arte e della guerra, scavando un solco nei confronti dell'umanità servile (il «gregge» dedito al bruto lavoro dei campi). Una civiltà dorata, dunque, proprio perché fondata sul principio gerarchico della distinzione. E in cui la classe sociale dominante, attraverso un lungo ma fecondo percorso evolutivo, ha raggiunto un alto livello spirituale, divenendo così gelosa custode di una supremazia conquistata nel corso di diverse generazioni. Tutto ciò è disprezzato e schernito nella scialba tavola dei valori borghesi, là dove conta soltanto la ricerca del profitto e la negazione di ogni possibilità del gesto eroico.

15 A margine della svolta nietzschiana: «I concetti di decadenza, di nichilismo, di trasvalutazione dei valori, di volontà di potenza, sono profondamente radicati nell'essenza della civilizzazione occidentale» (Raciti 2017, 54).

16 Questa la definizione proposta da Spengler in Anni decisivi (1933): «Il cesarismo perfetto è dittatura, non la dittatura di un partito, ma quella di un solo uomo contro tutti i partiti, prima di tutto il proprio» (cf. Evola 1973, 197). Segue la lode a Mussolini, considerato un autentico 'capo', paragonabile ai grandi condottieri rinascimentali. 
La storia, dunque, per Spengler (e d'Annunzio) più che lotta di classe è lotta di valori: un sordo scontro tra la subdola mediocrità e l'eccellenza aristocratica, infiacchita nell'inevitabile declino che tocca in sorte a tutte le manifestazioni mature della Zivilisation. Il filosofo tedesco, in questo contesto, lancia un vero e proprio anatema che conferisce veste filosofica, in prospettiva storicista, a ciò che in d'Annunzio era piuttosto una condanna dell'aridità borghese nemica della bellezza:

«La stola e la spada» si contrappongono all'aratro con la pretesa di caste che si oppongono al resto, alla non-casta, all'umanità che ha anch'essa uno stato, ma privo di un significato profondo. La distanza interna e sentita ha caratteri così fatali e possenti, che non v'e comprensione capace di superarla. Dai villaggi scaturisce un odio, che ha per controparte il disprezzo nutrito nei castelli. [...] Con la città, ma più tardi di essa, appare poi la borghesia, il «Terzo Stato». Anche il borghese guarda dall'alto la campagna che, ottusa, invariabile, mero oggetto della storia, si stende intorno a lui, sentendosi di fronte ad essa più desto e più libero, quindi più progredito sulla via della civiltà. Ma egli disprezza anche le caste originarie, «i feudali e la pretaglia», considerandoli come qualcosa che intellettualmente gli è inferiore e che storicamente ha lasciato indietro. Rispetto alle due caste originarie il borghese è però, non meno del contadino, un residuo, e il non-casta. (1143)

L'aristocrate è l'uomo quale storia, il sacerdote è l'uomo quale natu$r a$. La storia in grande stile è sempre espressione ed effetto dell'esistenza di una comunità nobile. (1149)

Inevitabile, per Spengler, che siffatta 'non casta' trovi nella metropoli il proprio spazio di vita ideale. Qui infatti tutte le identità sbiadiscono. Qui si annida il germe della decadenza che inevitabilmente corrode tutte le civiltà apparse nella storia. Qui l'individuo può scatenare una lotta cupa contro i suoi simili, fatta di intrighi e basse astuzie, ma soltanto al fine (vile) di soddisfare la volgare brama dell'arricchimento materiale. ${ }^{17} \mathrm{Si}$ eclissano così non solo le differenze fondate saldamente sul 'sangue', ma perfino le manifestazioni spirituali più alte dell'uomo.

La metropoli spengleriana somiglia perciò in modo indiscutibile alla «città infetta» da cui Claudio Cantelmo fugge inorridito. Ancor più nell'epoca in cui diventa impellente la questione del rapporto tra

17 «Le lotte assai più visibili che si svolgono nelle città tarde hanno un significato tutto diverso, poiché qui, come in ogni specie di comunismo, non si tratta di qualcosa di vissuto, bensì della proprietà concepita come quella di mezzi puramente materiali» (Evola 1991, 1160). 
'l'uomo e la tecnica'; in cui il soggetto, inchiodato alla sua condizione di «animale da rapina», deve occultare le sue pulsioni rapaci in obbedienza all'etica borghese, che ogni conflitto sposta sul versante economico. ${ }^{18} \mathrm{E}$ tutto questo, per Spengler come già per d'Annunzio, è indizio sicuro di profonda degenerazione. Di tramonto dell'umanità migliore e di aurora della mediocrità trionfante. Queste le parole del pensatore tedesco:

Vi è del cosmopolitismo sia all'inizio sia alla fine di una civiltà, ma nel primo caso perché la potenza simbolica delle forme castali sovrasta ancora le nazioni, nel secondo perché queste si dissolvono in una massa informe [...] La nobiltà è invero casta in senso proprio, è una sintesi di sangue e di razza, è una corrente dell'esistenza nella forma più perfetta immaginabile. (1145)

Ciò non significa affatto che il declino sia irreversibile. Perché nella storia, coi suoi corsi e ricorsi ciclici, nulla si disperde mai del tutto. Pia illusione è dunque quella dei sagaci borghesi approdati al potere, che pensano di essersi sbarazzati definitivamente delle scorie aristocratiche. Fatalmente, nuovi individui ravviveranno l'antica fiamma. E altri ceppi risorgeranno. Come aveva pronosticato d'Annunzio. ${ }^{19} \mathrm{E}$ come Spengler ipotizza, ponendo il cesarismo nel momento cruciale della decadenza, proprio quando, la civilizzazione ascende al suo momento culminante, avviando la consueta caduta à rebour:

Invece la civilizzazione - vero ritorno alla natura - è l'estinguersi della nobiltà non come ceppo (il che non avrebbe tanta importanza) bensì come tradizione [...] Della nobiltà allora non resta più che il nome; ora, proprio per questo la storia nella civilizzazione riguarda la sola superficie, essa si orienta verso scopi sporadici e vicini e, dal punto di vista cosmico, diviene informe, dipende dal caso che fa sorgere grandi individualità, restando priva di intima sicurezza, di linea, di significato. Col cesarismo la storia ridiscende nella sfera dell'astorico, nel ritmo primitivistico proprio alla preistoria e alle lotte, interminabili quanto insignificanti, per il potere materiale. (1150-1)

18 «Dal sentimento della potenza nascono la conquista, la politica e il diritto; dal sentimento della preda nascono il commercio, l'economia e il danaro» (Evola 1991, 1161).

19 «Infatti in ogni periodo primo questa ascesa creativa verso la forma vivente corrisponde a ciò che in ogni periodo tardo sarà la potenza della tradizione, cioé la vecchia e salda disciplina, il ritmo divenuto sicuro e talmente vigoroso che anche all'estinguersi degli antichi ceppi sa attrarre continuamente nella sua orbita, dal profondo, sempre nuovi uomini, sempre nuove correnti dell'essere. Non si può dubitare che la storia delle epoche più tarde nella sua forma e nel suo ritmo sia già compresa, potenzialmente ma irrevocabilmente, nelle prime generazioni» (cf. Evola 1991, 1150). 
La prospettiva ottimistica salda quindi la concezione dannunziana con quella spengleriana. Nel senso che proprio dal fondo più oscuro della degenerazione scaturirà il percorso di rinascita. Perché la storia, dialetticamente fatta di albe e tramonti, è scandita dal fatale riemergere di un'aristocrazia a cui spetta il compito di infliggere il colpo mortale alla civiltà borghese. Si rovescia in questo modo il noto paradigma di Marx e si intravede, al tramonto della civiltà borghese, l'avvento non del proletariato, ma di una nuova-antica aristocrazia:

la stessa nobiltà in senso storico è infinitamente di più di quel che si pensa nei periodi tardi di una vita comoda: non è una somma di titoli, di diritti e di cerimonie, ma un possesso interno difficile a conseguire e difficile a mantenere che, una volta che lo si comprende, appare già degno del sacrificio di tutta una vita. Una stirpe antica non significa soltanto una serie di antenati, perché tutti hanno degli antenati, bensì di progenitori che vissero all'altezza della storia durante generazioni e generazioni e che non solo ebbero un destino, ma furono anche destino: progenitori, nel sangue dei quali la forma, attraverso esperienze centenarie e un'adeguata disciplina, è divenuta perfetta. (1152-3)

D'Annunzio probabilmente non legge queste parole. Ma se lo avesse fatto se ne sarebbe certo compiaciuto. E avrebbe visto in Spengler un suo degno discepolo. 


\section{Bibliografia}

Andreoli, Annamaria; Lorenzini, Niva (a cura di) (1982-84). D'Annunzio, Gabriele: Versi d'amore e di gloria. Milano: Mondadori.

Andreoli, Annamaria; Lorenzini, Niva (a cura di) (1989). D’Annunzio, Gabriele: Prose di romanzi, vol. 2. Edizione diretta da Ezio Raimondi. Milano: Mondadori.

Andreoli, Annamaria (a cura di) (1996). D’Annunzio, Gabriele: Scritti giornalistici. Milano: Mondadori.

Andreoli, Annamaria (a cura di) (2013). D’Annunzio, Gabriele: Prose di Romanzi. Milano: Mondadori.

Andreoli, Annamaria (a cura di) (2013). D’Annunzio, Gabriele: Tragedie, sognie misteri. Milano: Mondadori.

Cacciatore, Fortunato (2005). Indagini su Oswald Spengler. Soveria Mannelli: Rubbettino.

Carducci, Giosué (1963). Giambi ed Epodi. Bologna: Zanichelli.

Carli, Mario (1920). Con D’Annunzio a Fiume. Milano: Facchi.

Colli Giorgio; Montanari, Mazzino (a cura di) (2011). Nietzsche, Friedrich: Così parlò Zarathustra. Milano: Adelphi.

Conte, Domenico (2009). Albe e tramonti d'Europa. Ernst Jünger e Oswald Spengler. Roma: Biblioteca di Letteratura.

Croce, Benedetto (1950). Pagine di guerra. Roma-Bari: Laterza.

De Felice, Renzo (1978). D’Annunzio Politico 1918-1938. Roma-Bari: Laterza.

De Felice, Renzo; Gibellini, Pietro (a cura di) (1987). D’Annunzio politico = Atti del convegno (II Vittoriale, 9-10 ottobre 1985), Quaderni Dannunziani, nuova serie, $1-2$.

Evola, Julius (a cura di) (1973). Spengler, Oswald: Anni decisivi. Roma: Il Borghese.

Evola, Julius (a cura di) (1991). Spengler, Oswald: Il Tramonto dell'Occidente. Lineamenti di una morfologia della storia mondiale. Parma: Guanda.

Gentile, Emilio (1996). Le origini dell'ideologia fascista (1918-1925). Bologna: il Mulino.

Giardina, Andrea; Vauchez, André (2000). Il mito di Roma. Da Carlomagno a Mussolini. Roma-Bari: Laterza.

Losurdo, Domenico (2001). La comunità la morte l'occidente. Torino: Boringhieri.

Mangoni, Luisa (1979). «Per una definizione del fascismo: i concetti di bonapartismo e cesarismo", Italia contemporanea, 135, 17-52.

Nordau, Max (2009). Degenerazione. Milano: Bocca.

Oliva, Gianni (a cura di) (1999). Lettere ai Treves. Milano: Garzanti.

Raciti, Giuseppe (a cura di) (2016). O. Spengler: L'uomo e la tecnica. Torino: Aragno Editore.

Raciti, Giuseppe (a cura di) (2017). O. Spengler: Il tramonto dell'Occidente. Torino: Aragno Editore.

Salaris, Claudia (2002). Alla festa della rivoluzione. Artisti e libertari con D’Annunzio a Fiume. Bologna: il Mulino. 



\title{
Stato, problemi, applicazioni critiche della filologia dannunziana
}

Elena Maiolini

Università Ca' Foscari Venezia, Italia

\begin{abstract}
The paper traces some general reflections on the problems, methods and perspectives of authorial philology applied to d'Annunzio's work and in relation to some recent scientific contributions. Following Contini's philological sensitivity, the d'Annunzio variant studies have dealt with a peculiar processing method that manifests itself almost instantly, in currenti calamo interventions. In critical editions, they are given an adequate representation through multilayered apparatuses and through the distinction between text's prehistory and protohistory. The critical applications of these studies have since been the most attractive prospects for an investigation into the elaboration of d'Annunzio's text.
\end{abstract}

Keywords D’Annunzio. Philology. Critical editions. Text prehistory. Text protohistory.

Sommario 1 Frutti recenti. - 2 Scartafacci.-3 Criterio topografico o criterio cronologico. - 4 Preistoria testuale. - 5 Applicazioni critiche della variantistica. - 6 Sul metro.

\section{Peer review}




\section{$1 \quad$ Frutti recenti}

Alcuni contributi recenti invitano a fare il punto sugli studi dedicati a D'Annunzio, il testo e la sua elaborazione, come Emilio Mariano, Pietro Gibellini e Eurialo De Michelis titolavano il contributo collettivo gardonese da cui prese avvio, oltre quarant'anni fa (era il 1977), la variantistica dannunziana (Quaderni del Vittoriale 1977). ${ }^{1}$

Nel 2013 sono stati pubblicati gli atti di una giornata in ricordo di Franco Gavazzeni coordinata da Gibellini. Il volume rende omaggio fin dal titolo (L'officina di d'Annunzio) all'impegno del fine studioso che «vagliò gli strumenti lessicografici usati da d'Annunzio, ne indagò le fonti antiche e le riprese moderne, procurò le concordanze alcionie, ricostruì la vicenda testuale delle opere, le corredò di commenti, recuperò testi inediti o dispersi...» (Gibellini 2013, XIV)². La sua lezione metodologica e interpretativa è ripercorsa dagli interventi di Gibellini ( «D’Annunzio, Pascoli e Marinetti di fronte al mito»), Cristina Montagnani («1903: un anno vissuto pericolosamente»), Raffaella Bertazzoli («Perizia metrica sul d'Annunzio tragico»), Clelia Martignoni («Appunti sulla prosa») e Gianni Mussini («Le lettere di Franco»), oltre che dalla recensione, riprodotta in appendice, con cui Gibellini (1981) salutò il libro sulle Sinopie di «Alcione» (Gavazzeni 1980) in Studi e problemi di critica testuale.

Recentemente è stata poi rilanciata, dopo qualche anno di silenzio dovuto a ragioni varie, l'attività dell'Edizione nazionale coordinata dal Comitato ora presieduto da Gibellini e composto da Raffaella Bertazzoli, Nadia Ebani, Clelia Martignoni, Pier Vincenzo Mengaldo, Cristina Montagnani, Giorgio Zanetti e dall'attuale presidente del Vittoriale, Giordano Bruno Guerri. La prestigiosa collana - che già annovera l'Alcyone a cura di Gibellini (1988), le Elegie romane a cura di Maria Giovanna Sanjust (2001), La Figlia di Iorio a cura di Raffaella Bertazzoli (2004), Maia a cura di Cristina Montagnani (2006) e la Fiaccola sotto il moggio a cura di Maria Teresa Imbriani (2009) - si è arricchita infatti dell'Elettra a cura di Sara Campardo (2017). Di Elettra si dispone della minuta autografa, che attesta la parte più sostanziosa e significativa dell'elaborazione testuale: rientrava dunque tra le opere sulle quali si concentra la nuova Edizione Nazionale, per la quale sono in preparazione le Cento e cento e cento e cento pagine del libro segreto di Gabriele d'Annunzio tentato di morire, la Contemplazione della Morte, la Francesca da Rimini, Il ferro, Il fuoco, La nave, La vita di Cola di Rienzo, Le vergini delle Rocce, il Notturno e il Sogno d'un mattino di primavera.

1 Per una testimonianza della collaborazione da cui nacque, tra le altre cose, anche quel convegno, si veda Gibellini 2008, 154.

2 Si veda a questo proposito Zava 2016, 168. 
Una nuova edizione critica dell'Alcyone è stata inoltre pubblicata nel 2018 nella «Collana di classici italiani» diretta da Cesare De Michelis e Gilberto Pizzamiglio per Marsilio, in co-edizione con l'Edizione Nazionale, in ragione del numero ragguardevole di autografi emersi tra il 1990 e il 2009 (ventuno minute e otto belle copie) che hanno reso necessario un aggiornamento (Gibellini 2018, 13-5). Il libro abbina l'apparato filologico di Gibellini a un commento poderoso (quasi 400 pagine, all'incirca metà volume) affidato alle tre studiose cafoscarine Giulia Belletti, Sara Campardo e Enrica Gambin (Gibellini 2018, 479-861), attento alle varianti, oltre che alle fonti e agli echi nella poesia del Novecento: «censisce e vaglia la vasta messe dei contributi precedenti, indicando, specie per l'individuazione delle fonti, la paternità del reperto», ed «estende i rinvii intertestuali al dopo-Alcyone, aggiungendo alle fonti usufruite dal poeta [...] gli echi dei versi alcionii rintracciabili nei principali poeti novecenteschi» (Gibellini 2018, 15). Completano l'edizione le schede metriche curate da Gianfranca Lavezzi (Gibellini 2018, 863-84).

L'anno scorso, infine, un libro su Come lavorava d'Annunzio (2018), scritto a quattro mani da Pierandrea De Lorenzo (capp. 1-4) e Cristina Montagnani (cap. 5), è uscito nella serie «Filologia d'autore» della collana «Bussole» di Carocci, diretta da Simone Albonico, Paola Italia e Giulia Raboni. In linea con le finalità della serie, che intende offrire un orientamento pratico nello studio di grandi autori, il libro inquadra la poetica, mappa gli archivi e presenta la modalità compositiva, con un approfondimento sull'articolarsi di un'opera, in questo caso le Laudi (nel capitolo che si deve a Montagnani). Particolarmente utili risultano i capitoli centrali, dove si presenta un panorama complessivo sulla dislocazione dei manoscritti e dei libri, tra il Vittoriale (l'Atto di donazione allo Stato pubblicato sulla Gazzetta Ufficiale del Regno d'Italia il 4 dicembre 1930, il ruolo di Antonio Bruers nell'organizzazione degli autografi e delle migliaia di volumi nella villa di Cargnacco, la composizione della biblioteca già appartenuta a Henry Thode e in seguito arricchita, ecc.), la Biblioteca Nazionale Centrale di Roma (il lavoro di Annibale Tenneroni, di Federico Gentili di Giuseppe e degli eredi, gli acquisti compiuti nella seconda metà del Novecento, ecc.) e l'Istituto per il Teatro e il Melodramma della Fondazione Giorgio Cini di Venezia (i copioni autografi delle tragedie di mano di Eleonora Duse, ecc.).

Questi contributi e frutti della filologia dannunziana invitano chi scrive, alle prese con l'edizione critica della Francesca da Rimini, a riflettere sui problemi, i metodi e le prospettive del cantiere. ${ }^{3}$

3 Vi attendo beneficiando di un assegno di ricerca cofinanziato dal Dipartimento di Studi Umanistici di Ca' Foscari e dal Vittoriale degli Italiani, sotto la guida di Michela Rusi e Pietro Gibellini (cf. «Notizie dell'Edizione Nazionale». Archivio d'Annunzio, 


\section{Scartafacci}

Contro l'entusiasmo per la critica delle varianti si sono affacciate perplessità che è opportuno tener presenti, come ha suggerito Franco Suitner (2012) in una sintesi penetrante - a cui si rimanda - dei problemi posti dalla cosiddetta 'critica degli scartafacci'. Dalle obiezioni fondamentali di Benedetto Croce (e poi dei crociani) circa l'Illusione sulla genesi delle opere d'arte documentabile dagli scartafacci degli scrittori (Croce 1948), secondo le quali l'opera d'arte è sintetica a priori e quindi non deve invischiarsi del retrobottega dell'autore (vi si troverebbero «inezie» che al più possono destare e soddisfare una certa «curiosità», ma non stimolare o sostenere una riflessione critica intera), si possono trarre indicazioni di metodo, spunti per un pragmatismo con cui allontanarsi dall'atteggiamento reverenziale implicito nelle rappresentazioni fotografiche, che obbligano il lettore a farsi filologo, o diplomatiche, spesso complicate e comunque non sufficientemente immediate.

Come si sa, Contini intervenne nella polemica, innescata dai suoi studi, con prudenza e in modo indiretto, rispondendo a Nullo Minissi, che nella rubrica «Noterelle e schermaglie» di Belfagor aveva espresso la sua sulla questione. Nel saggio «Come lavorava l'Ariosto» pubblicato nel Meridiano di Roma il 18 luglio 1937, indicava lo studio delle varianti come l'operazione più istruttiva, davvero pedagogica, da farsi con i manoscritti degli autori: esse, affermava, vanno considerate quali indicatori delle diverse fasi attraverso cui passa un testo, all'interno di un'esistenza dinamica; l'editore deve seguire il lavoro elaborativo e correttorio e rappresentarlo «in modo sùbito evidente, nella sua esatta cronologia» (Contini 1974, 233). Al modo statico di considerare l'opera, che si risolve in una descrizione topografica delle lezioni soggette a variante, Contini - e chi segue la sua scuola - preferisce un modo che indica una direzione per traguardi ideali (gli stati che conosce la poesia, dinamica come l'acqua) e tende a rappresentare cronologicamente la «vita dialettica» del testo:

Il primo [modo] stima l'opera poetica un «valore»; il secondo, una perenne approssimazione al «valore»; e potrebbe definirsi, rispetto a quel primo e assoluto, un modo, in senso altissimo, «pedagogico». È a questa considerazione pedagogica dell'arte che spetta l'interesse delle redazioni successive e delle varianti d'autore (come, certo, dei pentimenti e dei rifacimenti d'un pittore), in quanto esse sostituiscono ai miti della rappresentazione dialettica degli elementi storici più letterali, documentariamente accertati. (Contini 1974, 233-4; corsivi aggiunti)

5, 2018, 8, https://edizionicafoscari.unive.it/it/edizioni4/riviste/archiviodannunzio/2018/5/notizie-delledizione-nazionale/). 
Usciva in quegli anni anche la prima incursione Nell'officina di «Alcione» operata da Pietro Pancrazi (1939): lo studio sugli autografi, che il critico riproduceva in facsimile, poggiava sull'«elegante e fragile strumentazione del gusto», proponendo una lettura impressionista vòlta a dimostrare il progressivo miglioramento del testo (Gibellini 1985, 31). ${ }^{4}$ Con sensibilità di stampo continiano nasce la critica delle varianti dannunziana, il cui debutto andrà fissato nel 1973, quando al convegno su «D’Annunzio e il simbolismo europeo» Gibellini presentava il suo studio sui «Pentimenti della "Sera" fiesolana»: vi metteva in luce le linee correttorie della lirica, che facevano sistema, consegnando al sottotitolo, «Saggio di un commento alle correzioni di Alcyone», un omaggio proprio al Contini ([1943] 1970) del memorabile «Saggio di un commento alle correzioni del Petrarca volgare» (Gibellini 1976). ${ }^{5}$ Altre osservazioni generali avrebbe affidato ai saggi - riuniti poi nella prima parte di D'Annunzio dal gesto al testo (Gibellini 1995, 9-113) - premessi ai facsimili degli autografi di sette opere acquistate all'asta londinese della casa Christie's (Le vergini delle rocce, il ritratto di Cola di Rienzo, Il Vangelo secondo l'avversario e la Contemplazione della morte, il Ferro, i due Sogni): sostanzialmente, la tendenza a innalzare il registro linguistico-stilistico e a dilatare il testo, e la ripresa/l'occultamento delle fonti (la Cronica dell'Anonimo Romano trecentesco per La vita di Cola di Rienzo e i Vangeli apocrifi per Il Vangelo secondo l'avversario).

Non «una tecnica compiaciuta», ma «un'arte nitida e onesta», la filologia d'autore appariva anche a Gavazzeni - il 'Gava', nel ricordo di Mussini:

Perché la filologia non era per lui una tecnica compiaciuta ma un'arte nitida e onesta. Anche coraggiosa, non rinunciando mai al giudizio di valore: che è dopo tutto il motivo per cui si studiano i testi letterari. In questo Gava[zzeni] era naturaliter continiano, dunque inevitabilmente - se pur solo per certi aspetti - anche un po' crociano, poiché negli scartafacci cercava sempre le tracce profonde della poesia, la sua logica e il suo significato. (Mussini 2013, 52)

All'architettura del terzo libro delle Laudi Gavazzeni dedicò negli anni 1974-75 e 1976-77 i suoi corsi universitari pavesi, frequentati, tra gli altri, da Montagnani e Martignoni, che ne hanno tracciato un ricordo affettuoso e grato:

4 Lo studio su «La storia di "Alcyone"» era già apparso sui Quaderni del Vittoriale 1977, 67-103.

5 Il saggio è ora compreso, insieme a altri sulle correzioni alcionie, in Gibellini 1985, 85-117. 
Era il 1974, veramente del secolo scorso, pensando all'università di oggi; io avevo diciotto anni (il dato è del tutto irrilevante), quando mi trovai proiettata, forse meglio catapultata, nel cuore di una ricerca davvero al calor bianco: nuovi metodi, approccio diretto a materiali allora affatto sconosciuti. Seguire non era facile, e non era in ogni caso sufficiente: a ciascuno di noi venne affidato un tema seminariale, ovvero un malloppetto di autografi dannunziani fotocopiati, con qualche - vaga - istruzione per l'uso. Come avrebbe detto Gavazzeni, ce la cavammo «mica male». (Montagnani 2013, 20)

Le lezioni tenevano conto del contributo «Per la cronologia di Alcione» con cui Gibellini (1975) aveva studiato le varianti di struttura e il macrotesto, stabilendo la cronologia, prima ignota o mal nota, e spiegando perché d'Annunzio chiami «poema» la sua raccolta di liriche, dalla struttura piuttosto forte, aggiustata in corso d'opera. Dai corsi pavesi sarebbero geminati i due capitoli delle Sinopie, in cui Gavazzeni

esamina ogni spostamento interno, nella singola sezione come nel piano complessivo, ricostruendo lo svanire di certe sinopie e l'adempiersi di altre con o senza modificazioni, nell'affresco finale, ordinato sulle categorie distributive dello spazio e del tempo [...], che il critico distingue opportunamente nel loro valore ideale (la semanticità spazio-temporale del poema) e nel loro valore reale, genetico (i tempi e i luoghi di composizione). (Gibellini 2013b, 64-5)

In un'ottica in cui filologia e critica rimandano in modo circolare l'una all'altra, si capisce che la variantistica abbia una modalità pragmatica di pensare alla rappresentazione della dinamica compositiva, e quindi di costruire un apparato in cui, con le parole di Mussini:

il documento, il dato, non è dunque mai fine a se stesso ma sempre funzionale a un senso, in una filologia che - come nel Poliziano dell'Epistola aragonese - salva la parola per salvare la storia, la vita stessa. (Mussini 2013, 53)

Contini era parte del Comitato scientifico a capo dell'Edizione nazionale con cui nei primi anni Ottanta del secolo scorso il Ministero dei Beni Culturali decretò l'inizio di un nuovo corso all'Edizione, il cui Istituto era stato fondato il 21 giugno 1926, onore eccezionale per un autore ancora vivente. Presieduto da Dante Isella, il prestigioso Comitato era dunque composto da Contini, Domenico De Robertis, Franco Gavazzeni, Pietro Gibellini, Emilio Mariano, Pier Vincenzo Mengaldo, Giorgio Petrocchi, Ezio Raimondi e dall'allora presidente del Vittoriale, Egidio Ariosto. Compito del Comitato: guidare edizioni 
critiche che elaborassero scelte ecdotiche e rappresentative adeguate al peculiare metodo di lavoro di d'Annunzio, che con vena fluente

vergava a penna d'oca il testo sui fogli accuratamente tagliati, risolvendo man mano sui cartigli l'incepparsi della vena, l'ostacolo di una difficoltà locale. (Gibellini 1985, 50)

\section{Come spiega De Lorenzo, l’autore}

scrive i versi su fogli sciolti, e sulla scrivania ha poi altre carte, sempre gruppi di fogli sciolti, su cui prova e riprova un sintagma, risolve un verso o una rima inceppata, studia un giro di frase o di strofa. Carte quindi molto travagliate, sofferte, pasticciate, che il poeta, appena giunge a una redazione soddisfacente, getta nel cestino. [...]

In altri casi i filologi si imbattono invece in autografi che presentano correzioni e varianti interne. [...] Lo studio attento di queste carte, avviato ormai decenni fa, chiarisce molti aspetti del modo di lavorare dannunziano e, più in generale, del suo usus scribendi. Di solito d'Annuzio corregge currenti calamo, cosicché la prima lezione vergata subisce un intervento correttorio istantaneo e accoglie subito l'eventuale variante nel tessuto testuale. Non siamo quasi mai di fronte a correzioni che nascono da una rielaborazione avvenuta a distanza di tempo, o che sono il risultato di una modifica radicale della poetica sottostante, come ad esempio accade in Manzoni. (Montagnani, De Lorenzo 2018, 63)

Questa pratica è stata osservata da Gibellini per l'Alcyone e poi da tutte le curatrici delle edizioni critiche. Scriveva ad esempio Sanjust, introducendo alle Elegie romane, che la lirica «Elevazione» almeno in una prima fase

si concludeva dopo i primi nove distici, assai elaborati, seguiti da due coppie di tratti di penna sovrapposti e poi cassati quando l'autore decise di far succedere al motivo della nostalgia per la donna amata lontana, svolto nella prima parte, la spiritualizzazione della memoria sensuale (distici 10-16, che non mostrano lo stesso faticoso travaglio formale dei precedenti, proprio perché dettati da motivi shelleiani). (Sanjust 2001, XXVI)

Così Montagnani, chiedendosi «quali relitti di questa disperata foga elaborativa ci hanno conservato i manoscritti» di Maia, si rispondeva:

sono spesso individuabili tre distinti momenti redazionali: abbozzi del testo in fase nascente, tràditi da minuscoli foglietti, quasi relitti sospesi in un mare di riscritture occultate, carte che reca- 
no prime stesure già raffrontabili con il testo finale, e infine carte che registrano al tempo stesso prime stesure di alcuni versi e abbozzi dei versi immediatamente successivi. Documenti preziosi che ci rimandano l'immagine di un lavoro condotto senza soste. (Montagnani 2006, XXV)

Il riscontro è supportato dalla testimonianza di Tommaso Antongini sulle numerose ricopiature che precedono l'esemplare in pulito:

Il primo manoscritto, quello che si può chiamare l'originale, è tormentatissimo, irto di richiami, seminato di correzioni e di cancellature, in talune parti difficilmente decifrabile anche per chi abbia dimestichezza con la scrittura del poeta [...] Questa prima copia rimane per così dire invisibile a tutti. (Antongini 1938, 366 cit. in Montagnani 2006, XXV nota 36)

È quanto nota ora anche Campardo, che introducendo Elettra avverte come d'Annunzio lavorasse

su fogli sciolti in modo da poter agevolmente sostituire quelli troppo travagliati e poter far credere di aver scritto di getto, senza pause né ripensamenti. Veniva, però, 'tradito' dal suo segretario personale, Benigno Palmerio, veterinario di origine abruzzese promosso al rango di segretario, amministratore e confidente già ai tempi della Capponcina. Efficiente e fidato factotum, Palmerio si dimostra oggi preziosissimo per gli studi dei filologi dannunziani, perché aveva l'abitudine di recuperare e conservare le carte che d'Annunzio stracciava e gettava. Queste carte, contenenti appunti, abbozzi di versi, prime redazioni, risultano strumenti imprescindibili per comprendere la genesi di alcune liriche e, in generale, forniscono notevoli informazioni sul modo di lavorare del poeta abruzzese. (Campardo 2017, XXXI-II)

Quanto all'autografo della Francesca da Rimini, le 399 carte sciolte custodite presso la Biblioteca Nazionale Centrale di Roma (sezione Archivio Raccolte e Carteggi, nr. 5.I/C1), scritte tra luglio e settembre 1901 (le date si evincono dalle indicazioni poste in calce alle ultime pagine di ciascun atto), mostrano un travaglio elaborativo precedente a una ricopiatura in pulito di cui non resta traccia. Alcuni fogli sono talmente pasticciati da rendere alquanto laborioso lo sforzo di districare la successione dei ripensamenti. Si veda come esempio la sola carta 22 bis (la numerazione, progressiva, è dell'autore).

Considerando il ruolo marginale della copia dattiloscritta spedita a Georges Hérelle, che ne avrebbe predisposto la traduzione francese poi uscita a Parigi presso Calmann-Lévy nel 1913 (252 carte conservate nel Fondo Hérelle della Biblioteca di Troyes, nr. d'inventario 


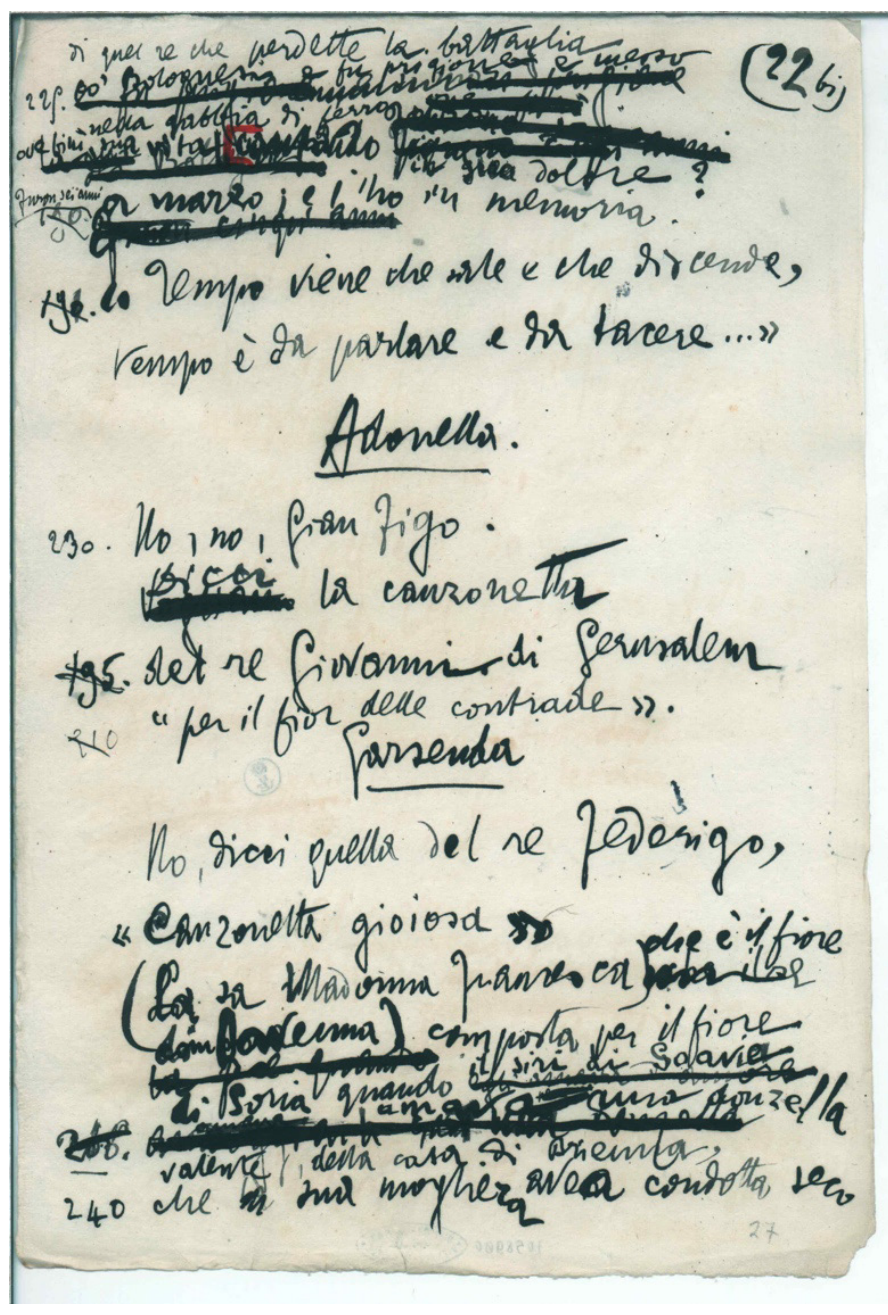

Figura 1 Gabriele d'Annunzio, Francesca da Rimini. 1901. Biblioteca Nazionale Centrale di Roma, ms nr. 5.I/C1, c. 22 bis. Riprodotta online in http://digitale.bnc.roma.sbn.it /tecadigitale/manoscrittomoderno/ARC5IC1/BNCR_DAN15514_029

3123), il manoscritto romano resta l'unico testimone della storia compositiva della tragedia, cui fanno da contorno gli autografi (in pulito o quasi) dei versi dedicati alla Duse composti a ridosso della princeps Treves, uscita a fine marzo 1902 (il manoscritto è datato «San Gabriele: 18 marzo 1902»), e del «Commiato», custoditi nell'Archivio Privato del Vittoriale (lemma 43, nrr. d'inventario 353-7 e 358-66). 


\section{Criterio topografico o criterio cronologico}

L'Alcyone con cui Gibellini inaugurò l'Edizione Nazionale fu impostato insieme al «maestro» Isella e al «maestro del maestro» Contini, al quale si deve la scelta e la definizione dell'apparato «algebrico» che rappresenta in modo accuratissimo la successione cronologica delle varianti (Gibellini 2018, 14). Assunsero le stesse opzioni teorico-metodologiche le edizioni che seguirono, compresa la nuova Marsilio, nella cui «Nota al testo» Gibellini richiama i due criteri-guida fondamentali:

1. le correzioni sono rappresentate con criteri cronologici e non topografici, cioè in base alla successione delle varianti e non alla loro posizione sul foglio; 2 . le varianti evolutive sono distinte da quelle sostitutive. (Gibellini 2018, 48)

La rappresentazione del lavoro correttorio con evidenza immediata e «nella sua esatta cronologia» è diretta emanazione delle esigenze continiane:

Nel rappresentare l'iter correttorio della minuta, privilegio, come detto, il criterio cronologico, trascurando quello topografico. In un'età in cui l'accesso alle riproduzioni fotografiche e digitali è quanto mai agevole, la resa diplomatica dell'autografo risulterebbe poco utile, e soprattutto non sufficiente a chiarire ciò che più preme: la successione temporale delle lezioni concorrenti. Nell'apparato, allestito con un occhio allo scorrere della penna e uno a quello del senso, cioè considerando sia la morfologia scrittoria sia la semantica delle varianti, ho seguito due criteri basilari: 1. distinguere le varianti evolutive da quelle sostitutive, ossia le lezioni interrotte da quelle formulate e rimpiazzate, poiché un aborto differisce da un bimbo morto anche nel reparto neonatale della filologia d'autore; 2. rappresentare la fase correttoria nel suo complesso, e non frammentarla in interventi isolati. (49-50; corsivi aggiunti)

La distinzione tra varianti evolutive e varianti sostitutive risponde a una riflessione sullo statuto delle correzioni, secondo la riflessione «del maestro del maestro»:

Le prime furono operate dal poeta all'istante lasciando in tronco la stesura del verso: le ho marcate con una barra verticale che suggerisce il senso di un blocco, di un arresto repentino. Le varianti sostitutive subentrano invece a lezioni consolidate, un periodo concluso, un verso ultimato, un sintagma compiuto ancorché breve: le ho contrassegnate con una freccia che rende l'idea di un transito. Dunque le varianti evolutive comportano il passaggio da un fram- 
mento a un testo, quelle sostitutive da un testo a un altro testo: per dirla con Contini, che riprendeva in chiave ecdotica un'antitesi crociana, dalla non-poesia alla poesia nel primo caso, nel secondo da uno «stato di poesia» a un altro, metafora attinta alla fisica dal geniale filologo. (50; corsivi aggiunti)

L'edizione del 2018 mostra in modo ancora più evidente il processo grazie alla scelta di porre la sequenza delle lezioni in stretto ordine cronologico, rinunciando alla parentesi quadra chiusa ( ] ) e alle didascalie che indicavano le tipologie di varianti:

Il nuovo apparato cambia la rappresentazione tipografica delle varianti. Nell'apparato del 1988 la lezione ne varietur soggetta a modifica era posta all'inizio, delimitata da una quadra chiusa, e era seguita dalle varianti, precedute dalle didascalie su e da, designanti rispettivamente le instaurative e le sostitutive. Ora i due segni sono stati rimpiazzati [...] da una barra verticale e da una freccia, e la ne varietur è posta a fine sequenza: le lezioni si susseguono così da sinistra a destra in aderenza alla loro successione cronologica. (51; corsivi nell'originale)

Si confrontino ad esempio le prime fasce degli apparati del 1988 e del 2018:

1-2] da Dolci sien le mie parole ne la sera | come il fresco fruscìo che fan le foglie $A$ - Fresche] Dolci $C$

1-2 Dolci sien le mie parole ne la sera / come il fresco fruscìo che fan le foglie $\rightarrow$ Fresche le mie parole ne la sera / ti sien come il fruscìo che fan le foglie

Già la prima impostazione consentiva una lettura intuitiva del percorso, e Montagnani può infatti esemplificare con chiarezza la prassi elaborativa proponendo un assaggio del primo apparato predisposto per gli autografi di Alcyone, in cui l'uso delle didascalie da e su rimarca la distinzione «capitale» tra le due tipologie di varianti:

Di solito [...] le correzioni di d'Annunzio furono operate currenti calamo, nel corso della stesura della lirica. Tale essendo il modo del correggere dannunziano, informazione essenziale che l'apparato genetico di Alcyone deve preoccuparsi di offrire è la distinzione tra varianti evolutive e varianti sostitutive: nelle varianti evolutive la lezione fu modificata prima che il dettato proseguisse conchiudendosi nell'unità del verso; nelle seconde invece una forma compiuta subentrò a una precedente forma altrettanto seppur provvisoriamente compita. La distinzione, capitale, è rimarca- 
ta dall'uso di due distinte abbreviature didascaliche, $d a$ (ricavato da) per le varianti sostitutive, su (corretto su) per quelle evolutive. (Gibellini 1988, XCVII; corsivi nell'originale; cf. Montagnani, De Lorenzo 2018, 126-31) ${ }^{6}$

L'impostazione procede secondo il modello dell'apparato negativo, ossia riportando accanto alla porzione di testo soggetta a variante, delimitata da una parentesi quadra chiusa, le lezioni divergenti precedute dalla didascalia che ne indica il tipo. La mantengono tutti i volumi dell'Edizione Nazionale, con alcuni scarti dovuti a esigenze peculiari, quali ad esempio un uso più scarso della parentesi quadra per l'edizione di Maia, e il ricorso a indicazioni topografiche tramite didascalie in corsivo: che la lezione introdotta si collochi nell'interlinea superiore o inferiore (spscr. $a$, stscr. $a$ ), anziché a fianco nei margini o negli a capo (ascr. a), è un'informazione fornita da Sanjust per le Elegie romane (Sanjust 2001, XLIII), da Bertazzoli per La figlia di Iorio (Bertazzoli 2004, LXXV), e da Montagnani per Maia (Montagnani 2006, XLVI), mentre vi rinunciano Imbriani per La fiaccola sotto il moggio e Campardo per Elettra.

I criteri rappresentativi della nuova edizione dell'Alcyone sono stati introdotti nell'Edizione Nazionale proprio a partire da Elettra. L'aggiornamento consiste sostanzialmente nell'introduzione di una barretta verticale ( | ) e di una freccetta $(\rightarrow$ ) a rimpiazzo delle didascalie da e su (Campardo 2017, XXXVII). In conseguenza di ciò è caduto anche il modello dell'apparato negativo - che registra, dopo il numero di verso e la lezione definitiva, delimitata da una parentesi quadra chiusa, le lezioni divergenti seguite dalle sigle dei testimoni - e la fascia si legge in modo ancora più immediato grazie a una raffigurazione cronologica, che riporta da sinistra a destra le lezioni primitive e quelle successive al ripensamento. Il criterio del tradizionale apparato negativo è mantenuto sia in Elettra sia nel nuovo Alcyone per la seconda fascia, in cui sono registrate le varianti tra i testimoni, manoscritti e a stampa. Per i primi versi de La sera fiesolana presi in esame poco sopra si ha, quindi:

\section{Fresche] Dolci $C$}

Il criterio cronologico seguito a partire da Elettra vale anche per l'edizione della Francesca da Rimini cui attendo, dove distinguo dunque le varianti evolutive (indicate con una freccia) dalle correzioni istantanee (contrassegnate da una barra verticale), che lasciano la scrittura in tronco: questo a favore dell'individuazione del punto di partenza e dello stadio intermedio che precedono la lezione finale, per 
una lettura più immediata e fruibile. Traggo un esempio dall'atto II, da una battuta di Paolo in risposta a Francesca, che l'ha rimproverato di non averla abbandonata «alla fiumana | perché mi si prendesse e mi volgesse | al mare» (2, 3, vv. 399-401):

Francesca, tanto

è crudele la vostra rampogna $\mathrm{e}$

tanto è dolce che il cuore mi si fende

e l'anima mia trista mi si sparge

nel suon di vostra voce che è sì strano $(2,3$, vv. 408-12)

408-10 Francesca, troppo / è crudele la vostra / rampogna e troppo è dolce $\rightarrow$ Francesca, tanto / è crudele la vostra rampogna e / tanto è dolce che il cuore mi si fende

411-2 e l'anima si sparge nella vostra / voce e non so se soffra o se gioisca / o se voglia morire. $\rightarrow$ e l'anima mia trista mi si sparge / nel suono della vostra voce che è / sì strano. $\rightarrow \mathrm{e}$ l'anima mia trista mi si sparge / nel suon di vostra voce che è sì strano.

I versi conoscono alcune riformulazioni apprezzabili con chiarezza attraverso una rappresentazione complessiva, anziché frammentaria. Poco importa conoscere la collocazione topografica delle lezioni, scritte nell'interlinea o sovrascritte a una cassatura: la rappresentazione cronologica rende adeguatamente conto dell'elaborazione, mostrando in modo fluido, ad esempio, la rinuncia agli enjambement tra aggettivo possessivo e nome («la vostra | rampogna», «nella vostra | voce»).

Il criterio cronologico mostra meglio anche la natura speciale e sistematica del comporre dannunziano, messa in luce fin dal convegno del 1977: un'elaborazione

sempre tanto sollecita e determinabile in tempi ravvicinati e brevi, e, in ogni caso, [...] presso che sempre definibile in termini più quantitativi che selettivi (Bàrberi Squarotti 1977, 22)

un correggere che

è sempre espansivo, ampliando il periodo con giunte interlineari o marginali, più spesso con la dilatazione paratattica del periodo. (Gibellini 1977, 79; 1985, 50)

Ė quanto si riscontra anche per la Francesca da Rimini, come si può osservare da questi esempi: 
E noi tenemmo

questo rosaio

per benedetto; lo tenemmo intatto

come una roba di verginità;

né giammai ne fu colta

alcuna rosa [1, 5, vv. 992-997]

per benedetto; né mai ne fu colta / alcuna rosa $\rightarrow$ per benedetto; lo tenemmo intatto / come una roba di verginità [agg. interl.] / né giammai ne fu colta [agg. interl.] / alcuna rosa (vv. 994-997)

e per tutte le fonti,

che ridono e che piangono,

ne' luoghi ch'io non so,

mi pare sparso il mio valore [1, 5, vv. 1141-1144]

che ridono ne' luoghi ch'io non so $\rightarrow$ che ridono e che piangono [agg. interl.] / ne' luoghi ch'io non so (vv. 1142-1143)

Paolo porta una lunga e ricca sopravveste che gli scende più giù del ginocchio, fin quasi al collo del piede, stretta ai fianchi da una cintura gemmata per cui passa un bel pugnale dommaschino. La capellatura increspata, non ispartita su la fronte ma confusa e folta, gli ombra il viso come una nube. [didascalia d'apertura di 4, 4]

DID La capellatura increspata gli ombra il viso come una nube. | La capellatura increspata, non ispartita su la fronte ma confusa e folta, gli ombra il viso come una nube.

L’amante la bacia e ribacia insaziabile. [didascalia a 5, 4, v. 309]

DID L'amante... insaziabile agg. interl. con la bacia, insaziabile | la bacia e ribacia insaziabile

\section{$4 \quad$ Preistoria testuale}

La storia interna all'elaborazione dei versi, che per d'Annunzio si risolve in una stesura di getto, rapida quando abbia trovato il ritmo, fatto salvo per alcuni inceppamenti risolti in prove di versificazione su foglietti poi gettati (o eventualmente salvati da Palmerio), non esaurisce la vicenda creativa. Parlando della tecnica scrittoria, Gibellini chiarì la distinzione teorica tra preistoria e protostoria del testo:

Dietro questa rapida storia testuale c'è una nutrita preistoria o protostoria: il sobrio appunto tematico, le liste botaniche, l'appa- 
rato dei dizionari (la Crusca, il Tommaseo, il Forcellini, il marino dei Guglielmotti...): percorsi con facilità agevolata a volta dall'orecchio (quante associazioni nascono dalla consultazione sul Tommaseo di una voce imposta per sola virtù d'assonanza). E poi le fonti, richiamate talvolta dai dizionari ma poi attinte direttamente; e i libri - Régnier o Pascoli, Dante o Carducci - schiusi sullo scrittoio o memorizzati che siano, pongono il problema delle fonti (troppo datata la polemica sui plagi) in modo vario: presenza attiva e attivante, ora inconsapevole, ora lessicalizzata senza più relazione col contesto di provenienza, ora deliberatamente evocate con un diagramma che va dal virgolato alla citazione, al gioco del remake, all'indistinta allusione di un clima - fatto di più nomi e di più testi - non focalizzabile con nitore nei suoi ingredienti. E poi i taccuini. (Gibellini 1985, 50-1)

In un recente contributo per Ermeneutica letteraria, egli sostiene che la critica genetica (fondata sui materiali preparatori, cioè sulla preistoria del testo) chiarisce solo in parte la genesi di un testo, che

resta spesso insondabile, attestata com'è da testimonianze esterne e dunque indirette: quelle in cui l'autore manifesta un'intenzione compositiva, o altri ne riferisce, o in cui, come fa d'Annunzio, dichiara di aver composto 'mentalmente' una poesia

mentre la critica variantistica interpreta tutta l'elaborazione di un testo:

la preistoria testuale concerne la genesi mentale della lirica o la predisposizione di repertori tecnici (si pensi agli elenchi di rime o alle liste di vocaboli tecnici, come quelli attinti a dizionari marini o botanici utilizzati in varie liriche), mentre la storia testuale mostra il divenire della lirica attraverso successive opzioni stilistiche. (Gibellini 2014, 123)

Come spiega De Lorenzo, approntando l'edizione critica dell'Alcyone Gibellini precisò che

i confini fra la preistoria e la storia del testo corrono lungo il crinale che divide i materiali dell'avantesto da quelli della prima stesura. Si potrebbe quindi individuare una storia testuale - fatta di correzioni interne alla redazione autografa, di varianti fra i testimoni manoscritti e fra le stampe - che ha una sua preistoria negli elenchi di titoli, note lessicali, appunti, abbozzi tematici di varie poesie, progetti compositivi, passi di taccuini, repertori tecnici, ovvero tutto il materiale eterogeneo in parte già nato in funzione dell'opera, in parte autonomo. Avremmo da un lato la 'genesi men- 
tale' dell'opera e dall'altro il divenire del testo attraverso le varie fasi di definizione stilistica. (Montagnani, De Lorenzo 2018, 72-3)

Capita infatti che dalla penna di d'Annunzio qualcosa nasca come una cellula staminale trapiantabile, materiale che resta a disposizione della creatività letteraria come una traccia luminosa. È il caso degli spunti provenienti dal Vocabolario marino e militare di Alberto Guglielmotti, dal Dizionario geografico, fisico, storico della Toscana di Emanuele Repetti, dal Lexicon e dell'Onomasticon di Egidio Forcellini, del Prodromo della flora toscana di Teodoro Caruel, dalla Crestomazia italiana di Ernesto Monaci (con cui d'Annunzio aveva studiato a Roma)... e dai passi annotati nei preziosissimi (anzi, «prodigiosi», come li ha definiti Martignoni 2013, 45) taccuini su cui lo scrittore appunta le prime impressioni di motivi lirici:

fogli scritti a penna o a matita, che riportano appunti stesi con il supporto di un piano d'appoggio o, il più delle volte, annotati con un tratto di lapis veloce durante un viaggio in treno, in automobile, o in piedi davanti a un luogo che lo interessa, finanche in trincea durante gli anni della Grande guerra. (Montagnani, De Lorenzo 2018, 21)

Il loro rinvenimento diede un impulso molto forte allo studio dell'elaborazione testuale: essi sono infatti, scrisse Giorgio Bàrberi Squarotti in occasione del convegno collettivo di variantistica:

la forma più clamorosa non già di autobiografismo esasperato [...] bensì di riduzione della vita, della propria vita come quella di tutti coloro che vengono in contatto con lui, in primo luogo le donne, per l'edificazione dell'opera (Bàrberi Squarotti 1977, 14; corsivi aggiunti)

sono espressione di una fame onnivora che si manifesta nell'appropriazione tanto di esperienze esistenziali quanto di elementi letterari e di forme artistiche, antichi e contemporanei, provenienti dall'Italia o dall'estero, col fine di creare una nuova trasfigurazione, un altro sviluppo.

Della portata enorme di questi piccoli quadernetti che conservano la storia sommersa dei testi rendono adeguatamente conto gli exempla proposti da De Lorenzo:

Quando questi appunti confluiscono nell'opera letteraria trascinano con sé un intarsio di dati reali ed elementi immaginari che hanno l'identico peso nel momento dell'oggettivazione lirica o narrativa; ciò dà vita a straordinari paradossi creativi, per cui è difficile segnare un confine tra il vissuto e l'immaginario, là dove tutto 
prende vita con uguale dignità in una dimensione nuova. (Montagnani, De Lorenzo 2018, 23)

I Taccuini e poi gli Altri taccuini furono pubblicati nel 1965 e 1976 nella collana di «Tutte le opere di Gabriele D’Annunzio» dei «Classici Contemporanei» Mondadori diretta da Egidio Bianchetti, curati dalla figlia Enrica, cui si affiancò Roberto Forcella per il primo volume. I due volumi mondadoriani sono uno strumento di lavoro importante; nella giornata di studi in ricordo di Gavazzeni, Martignoni (2013, 48) ne ha auspicato un'edizione criticamente affidabile:

Chi abbia perlustrato anche superficialmente i taccuini in archivio, o anche solo letto con debita attenzione filologica i pur meritori apparati delle due edizioni Bianchetti (dove continuano a essere preziosi i riscontri con le opere) non può che desiderare una ricognizione del materiale più precisa e meno precaria, per capirne meglio la tipologia e la classificazione.

Alcune primizie «Dal taccuino inedito dell'“Alcione"» furono pubblicate da Isella nel 1972 in Strumenti critici, rivista nata in quegli stessi anni (1966) su iniziativa del trio pavese di Maria Corti, Isella e Cesare Segre, insieme a D'Arco Silvio Avalle; e delle tracce creative rinvenibili in quei quaderni si discuteva nel 1977 al convegno su «D'Annunzio, il testo e la sua elaborazione». ${ }^{7}$ Furono però impiegati per la prima volta come veri e propri materiali genetici, relativi alla preistoria testuale, nell'edizione critica di Alcyone, dove la complessità della diacronia variantistica fu risolta con un apparato a una 0 più fasce e con la distinzione tra preistoria e storia testuale, affidando all'appendice gli elenchi di titoli; le liste lessicali e gli appunti botanici; i 'motivi', gli abbozzi e appunti tematici; i passi dei Taccuini stesi tra il 1881 e il 1903 con materiale rielaborato nelle liriche (Gibellini 2018, 419-77).

\section{Applicazioni critiche della variantistica}

Studiando Alcyone come poema organico, Gibellini osservò come i ritocchi facevano sistema, consolidando l'antropomorfismo della natura, umanizzando pateticamente vegetali e astri. Da tale prospettiva, in cui filologia e critica rimandano circolarmente l'una all'altra, proviene l'invito a non limitarsi allo studio delle microvarianti, interessanti per le considerazioni puntuali che permettono, spingendosi a considerare la struttura dell'opera nella sua interezza, collocando 
quindi l'insieme delle correzioni all'interno di una storia elaborativa globale e rispondendo al compito di «tradurre in maniera formalizzata questo complesso divenire testuale» (Montagnani, De Lorenzo 2018, 73).

Alla definizione delle strutture dei suoi libri d'Annunzio approda dopo essersi chiarito un percorso. Ė esemplare il caso delle Laudi, cui lavora spartendo in modo travagliato temi e motivi finché, dopo la pausa del 1902 in cui si dedica a Il fuoco e alla Francesca da Rimi$n i$, il canzoniere entra in una fase di «profonda crisi strutturale» ripercorsa da Montagnani nella giornata di studi in onore di Gavazzeni: d'Annunzio la supera definendo un cammino temporale e spaziale attraverso il quale raggiunge il «risolutivo trionfo della modernità» sull'antico e sul classicismo, e prende congedo dalla Grecia (Montagnani 2013, 25-6). Nuove considerazioni sulla struttura delle Laudi sono avanzate da Campardo per Elettra, tradizionalmente considerata una silloge scissa, non compatta, soprattutto in rapporto a Maia e Alcyone: susciterebbe un'«impressione di frammentarietà» tutt'al più «attutita» dall'«apporto mitico» comune ai testi (Scicchitano 2011, 164). La posizione sulla quale si attestano i critici è ripresa da Montagnani, la quale toccando l'argomento afferma che Elettra, pubblicata nel dicembre 1903, contemporaneamente a Alcyone, in realtà

tradisce una natura intimamente composita, scissa fra i testi di carattere eroico e celebrativo, di impronta carducciana, e la sezione delle Città del silenzio. In poche parole, è già un testo residuale, alla cui articolazione d'Annunzio, in sostanza, dedicò poco impegno. (Montagnani 2013, 23)

Campardo avanza invece la tesi che la raccolta sia non l'esito dell'aggregazione di momenti lirici isolati, ma un libro strutturato con una marcata progettualità. ${ }^{8}$

Le applicazioni critiche sulla struttura complessiva delle opere provennero dalla variantistica dannuziana fin dagli esordi. Dall'entusiasmo per lo studio degli autografi degli anni Settanta fiorirono contributi preziosi come quello di Martignoni (1977) «Sull'elaborazione delle Faville del maglio» e di Ivanos Ciani sulle Novelle della Pesca$\mathrm{ra}$, uno dei pochi rifacimenti complessivi rispondenti a una variazione profonda della prospettiva poetica, ${ }^{9}$ cui lo studioso dedicò un libro

8 Si veda su questo Campardo 2017, liii-lxxxvii, § «La genesi del libro».

9 Tra i pochi altri, la raccolta di liriche Canto novo, di cui si ripercorre la storia in Montagnani, De Lorenzo 2018, 75-81: uscita nel 1882, sarebbe stata ripubblicata nel 1896 in una nuova edizione articolata in due sezioni, in cui all'accentuazione del panismo metamorfico si accompagna l'eliminazione dei sonetti di ispirazione sociale prima dominanti; è l'edizione che d'Annunzio sceglie per l'Edizione Nazionale del 1929, retrodatandola però al 1881 . 
pubblicato con Ricciardi nel 1975. Pubblicate nel 1902 presso Treves ma composte tra il 1884 e il 1888, esse uscirono con in calce le date «1884-1886»: privo di una visione storicistica («Tutta la vita è senza mutamento»), d'Annunzio desidera proporre con l'edizione complessiva delle sue novelle il primo assaggio di un'arte ormai sotto gli occhi di tutti. Sottopone dunque i testi a una riorganizzazione volta a

far scomparire dalle pagine giovanili quanto avrebbe potuto farle ritenere sentita adesione ai discreditati canoni del naturalismo, mentre, invece, io credo, altro non erano se non sudate carte di ricerca stilistica, e, al contempo, nobilitarle stilisticamente, sia perché il carattere di sudate carte vi risaltasse con maggiore evidenza, sia perché i nuovi lettori, insistentemente avvertiti nella prefazione della «puerilità» delle novelle presentate, si meravigliassero di trovarvi, al contrario, i segni di un'arte già non marginale alle sue prime manifestazioni. (Ciani 1975, 144; corsivi nell'originale)

Applicando la variantistica all'ermeneutica letteraria, Ciani studiò poi i rifacimenti del Piacere, pubblicandone nel 1976 con Il Saggiatore un'edizione «nella stesura preparata dall'autore per l'edizione francese del 1894», come recita il sottotitolo. Vi analizzava le diverse redazioni illustrando i rimaneggiamenti sostanziali operati in vista della traduzione di Hérelle, apparsa prima nella Revue de Paris tra dicembre 1894 e marzo 1895 e poi, con ulteriori modifiche, nel libro dell'editore parigino Calmann-Lévy (1895) (Ciani 1976, VII-XII). ${ }^{10}$ Soppressioni e sostituzioni tendono a sottrarre il romanzo da una lettura complice dell'«egoistica sensualità» e del «vano estetismo» di Andrea Sperelli (Papponetti, Cappellini 2001, 293); accanto a questo, si riscontra l'intenzione di ridimensionare i flashback e, soprattutto, di occultare le fonti francesi manifeste nei passi espunti, come si può osservare oggi anche nello schema con le «Fonti interne e fonti esterne» del romanzo di Ilvano Caliaro (1995), in appendice all'edizione Garzanti del Piacere allestita con Gibellini.

I lavori di Ciani sono tra i pochi in cui la filologia d'autore si sia applicata alla produzione narrativa, come nota Martignoni auspicando la ripresa dei molti «problemi critici e talora filologici ancora aperti per la prosa» (Martignoni 2013, 49). Tra le molte analisi che si potrebbero svolgere sulle strutture e sullo stile del d'Annunzio narratore (ai cui Volti e risvolti - ricordo per inciso - Ilaria Crotti (2016) ha dedicato il recente Lo scrittoio imaginifico, studiando in particolare la stagione 'notturna'), ritengo particolarmente interessante quelle

10 Si vedano inoltre «Premesse per uno studio sul Piacere» e «'Il piacere' francese» (Papponetti, Cappellini 2001, 245-52 e 253-305). 
che indica Martignoni richiamando l'attenzione sull'indagine intorno alle «Figure ritmico-sintattiche della prosa dannunziana» avviata da Gian Luigi Beccaria (1975). Mi pare infatti che si rivelerebbe incisiva un'analisi delle strutture melodiche che rivestono anche la sintassi di un lettore di Tommaseo dall'orecchio assoluto come il Nostro.

Un'altra applicazione critica è certamente l'indagine delle fonti, manifeste o occultate che siano. Gibellini riconosceva ad esempio nel Prodromo della flora toscana di Caruel custodito al Vittoriale (1860), «la fonte della flora di Alcyone», come recita il sottotitolo del saggio «Fiori di carta» incluso in Logos e mythos, interessante non solo per l'individuazione sicura della fonte, ma anche e soprattutto per le osservazioni circa «i modi del procedimento elaborativo dannunziano» (Gibellini 1985, 136): d'Annunzio parte da una pagina, più in particolare da una voce del libro aperto sullo scrittorio, e ne compendia i nuclei essenziali in fogli di appunti che confluiscono nella preistoria del testo, ritessuta in versi che ne fermano l'essenza. I contatti stretti tra le nozioni specialistiche del Caruel e le tessere botaniche della poesia dannunziana passano spesso attraverso il lessico del Dizionario della lingua italiana Tommaseo-Bellini, in cui altre contiguità vocabolaristiche gli consentono prelievi e appoggi più ampi prima dell'esito poetico. Traggo un solo assaggio dagli exempla forniti da Gibellini. Il Caruel tratta gli avorni con queste parole:

Fraxinus Ornus. Detto volgarmente Ornello, Orniello, Avorniello, Nocione ecc.

Fraxinus excelsior. [...] nei monti [...] all'Avernia in Casentino [...]

Cytisus alpinus. In montagna come il precedente (Cytisus Laburnum) ma solamente nella regione del faccio e dell'abeto: [...] e nel Casentino dov'è comune, per esempio sopra la Lama e all'Alvernia. Fior. in giugno e luglio. Frutt. in agosto. Tanto questa specie quanto la precedente sono note ai montagnoli col nome di Avorno, Avorniello, Avorniola e simili; mentre i campagnoli delle vallate la conoscono meglio sotto il nome di Maggiociondolo. (Gibellini 1985, 139)

I tratti essenziali della pagina scientifica sono riassunti da d'Annunzio in un foglio:

Nei monti dell'Alvernia (nel Casentino), sopra la Lama, fiorisce l'avorno (avornetto, avorniola) (giugno e luglio) fruttifica in agosto [vene]

Nel Tommaseo-Bellini il poeta reperisce un'auctoritas quattrocentesca per i nomi volgari delle piante: 
Avornio e Avorniello. Poliziano. Stanze 83 «L'avornio tesse ghirlandette al maggio». (Gibellini 1985, 139)

E il lacerto migra ai versi 41-42 de «L'Asfodelo»:

e su pe' monti della Verna

l'avornio tesser ghirlandette al maggio (Gibellini 1985, 139)

Nei versi, «la correzione ben leggibile nell'autografo [della Verna succede a dell'Alvernia] scopre un tentativo di occultamento della fonte botanica a vantaggio di quella letteraria» (Gibellini 1985, 139-40). «L'Asfodelo» è il testo che sblocca d'Annunzio permettendogli la ripresa della composizione di Alcyone nell'estate 1902, perché attraverso il calendario della fioritura suggerisce l'asse strutturale del poema: i materiali botanici dunque non solo illuminano le fonti, ma spiegano ad esempio la genesi di un testo-chiave prima sottovalutato.

Numerosi casi di questo procedimento offre anche la vicenda testuale della Francesca da Rimini, la cui stesura nell'estate 1901 s'incrocia alla composizione delle Laudi, avviata tra giugno e luglio 1899, ripresa in luglio e agosto 1900 e quindi nell'estate 1902, prima di essere portata a termine nell'estate 1903. Lo studio delle fonti e dei passaggi elaborativi della tragedia può approfittare oggi del commento curato da Donato Pirovano per una nuova edizione dell'opera uscita nel 2018 presso l'editore Salerno. ${ }^{11} \mathrm{Il}$ Tommaseo-Bellini è anche per la tragedia un passaggio fondamentale da cui d'Annunzio trae tessere riformulate in poesia. Così la citazione secentesca cui rimanda la voce "sellare», da Sopra il governo e il servizio della Cavalleria di Lodovico Melzo,

Devono gli offiziali andar per il quartiero svegliando i soldati, e ordinando loro che s'armino di petto e schiena, e che sellino i cavalli ${ }^{12}$

torna utile al poeta per una battuta di Paolo a Gianciotto, nell'atto quarto $(4,4$, vv. 507-508):

Ho visto che tua gente s'è armata di petto e schiena, e aspetta il buttasella.

e sella già i cav[alli] | e aspetta i g | e cinghia già la sella; / Giovanni. | e aspetta il buttasella. (508)

11 Si veda a questo proposito la recensione di Giallombardo (2018).

12 s.v. «sellare». Tommaseo, Niccolo; Bellini, Bernardo Bellini, Dizionario della lingua italiana. 4 voll, 8 tt. Torino: Unione Tipografico-Editrice, 1861-79. 
La fonte marcata dei versi («che s’armino di petto e schiena, e che sellino i cavalli» del Melzo), di cui avverte Pirovano, è tanto più evidente se si osserva il manoscritto con le correzioni d'autore, in cui il secondo elemento della prima stesura «di petto e schiena, e sella già i cav[alli]» è variato in «e aspetta i g[...]», «e cinghia già i cavalli», fino al definitivo «e aspetta il buttasella», con un evidente allontanamento graduale della fonte.

Similmente, il sonetto attribuito a Enzo, figlio naturale di Federico II, re di Sardegna dal 1238 e caduto prigioniero dei bolognesi nel 1249, che d'Annunzio legge nella Crestomazia italiana dei primi secoli di Ernesto Monaci, con cui aveva studiato a Roma (è piegato l'angolo della pagina corrispondente nel volume conservato nella Prioria, tra molti altri segni di lettura, note manoscritte e cartigli):

Tempo vene ki sale e ki discende, tempo è da parlare e da taciere, tempo è d'ascoltare e da imprendere, tempo è da minaccie non temere; tempo è d'ubbidir ki tti riprende, tempo di molte cose provedere, tempo è di venghiare chi tt'affende, tempo d'infignere di non vedere. Però lo tengo saggio e canoscente que' ke fa i fatti con ragione e col tempo si sa comportare, e mettesi im piacere de la gente, ke non si trovi nessuna cagione, ke lo su' fatto possa biasimare.

(Monaci 1889, 203-4)

si ritrova citato - Pirovano lo segnala - in una battuta di Biancofiore al primo atto (1, 1, vv. 319-321):

Intanto

racconta a noi! Siam tutte orecchi. «Tempo

è d’ascoltare» disse il prigioniero.

E di nuovo la correzione visibile sul manoscritto, dove il terzo verso termina in «lo re vinto», rende conto di una variante che va in direzione dell'allontanamento (se non, in questo caso, dell'occultamento) della fonte:

Intanto... prigioniero. agg. nel marg. sinistro, con Ti stiamo a udire: «È tempo / d' | Siam tutte orecchi: «Tempo / è d'ascoltare» - di$\mathrm{ce} \rightarrow$ disse - lo re vinto. $\rightarrow$ il prigioniero. (vv. 319-321) 
Un altro esempio: il verso

Dentro nel cuore mi ferì, ovunque è vena

che d'Annunzio legge in nota a una pagina dei Canti Greci di Tommaseo, terzo dei quattro volumi dei Canti popolari toscani corsi illirici greci pubblicati tra il 1841 e il 1842, di cui una copia si conserva al Vittoriale $^{13}$ insieme alle parole del commentatore, «Il fegato agli antichi era sede degli appetiti men nobili; de' più nobili il cuore», è rielaborato per una battuta di Garsenda, dama di compagnia di Francesca, che vedendo le lacrime della sua signora dice:

Dentro nel cuore

subito la ferì. Ah, s'ella è bella,

egli è pur bello, il Malatesta!

$(1,5$, vv. 1058-1060)

La ripresa passa ancora una volta da una prima stesura radente alla fonte, alla sostituzione 0 , in questo caso, alla soppressione di un emistichio («ovunque è vena»), in modo da allontanarsi dal testo di partenza:

Dentro nel cuore la ferì, ovunque / è vena. S'ella è bella, $\rightarrow$ Dentro nel cuore / sùbito la ferì... ovunque è vena. / Ah, s'ella è bella, $\rightarrow$ Dentro nel cuore / sùbito la ferì. Ah, s'ella è bella, / egli è pur bello (vv. 1058-1060)

L'operazione funziona anche con i recuperi petrarcheschi. Nell'atto quinto, Paolo esclama:

O mia vita, non fu mai tanto folle

il desiderio mio di te. Sentivo

già venir meno

dentro al core gli spiriti

che vivono degli occhi tuoi. La forza

mi si perdeva nella notte

$(5,4$, vv. 266-271)

Pirovano avverte del contatto con «Io sentia dentr'al cor già venir meno | gli spirti che da voi ricevon vita» del canzoniere di Petrarca (47, vv. 1-2), ma il verso 270 conosce una rielaborazione di cui testimonia il manoscritto:

13 Si cita da Maiolini 2017, 181 nota 75. 
270 che vivono di te. Tutta la forza $\rightarrow$ che vivono degli occhi tuoi. La forza

Dal «da voi ricevon vita» petrarchesco, si passa al «vivono di te» della prima stesura, a «vivono degli occhi tuoi» della seconda, con allontanamento dalla fonte a parità di risultato metrico grazie all'espunzione di «Tutta».

\section{Sul metro}

Alcuni degli ultimi contributi della filologia dannunziana invitano a ulteriori indagini sui fili ritmici con cui il poeta tesse i suoi versi: le schede metriche a cura di Lavezzi che chiudono la nuova edizione critica dell'Alcyone e la «Perizia metrica sul d'Annunzio tragico» proposta da Bertazzoli nella giornata di studi in onore di Gavazzeni (Gibellini 2018, 863-84; Bertazzoli 2013). Entrambe sono debitrici di saggi degli anni Settanta e Ottanta: di Gavazzeni, alla cui «Perizia metrica della "Figlia di Iorio"» presentata nel 1985 al convegno sulla tragedia organizzato da Edoardo Tiboni al Centro Studi Dannunziani pescarese, allude il titolo di Bertazzoli, e delle analisi strofiche alcionie poi comprese nelle Sinopie, cui rimanda Lavezzi (Gavazzeni 1986): $i^{14}$ ma anche di Mengaldo, che definì i meccanismi di scomposizione e ricomposizione per cui la poesia novecentesca è debitrice a d'Annunzio in due studi capitali del 1966 e 1972, poi riuniti nel libro su La tradizione del Novecento (1975). ${ }^{15}$

Richiamo l'attenzione su due questioni, tra le molte messe in luce. Innanzitutto la «cellula ritmica» della «Pioggia nel pineto» riscontrata da Lavezzi, che sulle presenze del componimenti «nel Novecento e oltre» è tornata l'anno scorso anche nell'articolo «Nuove gocciole dal bosco dannunziano» per Archivio d'Annunzio (Gibellini 2018, 869; Lavezzi 2018). Nella relativa scheda metrica dell'edizione alcionia, la studiosa individua nel ternario la misura ritmica più piccola del testo la quale, sdoppiata nel senario o triplicata nel novenario (il metro si ravvisa anche in forma scomposta: «che l'anima schiude | novella», vv. 27-8), ne determina il ritmo anapesto-dattilico, oltre che le rime e le assonanze interne.

E poi il «modulo strutturale» dell'endecasillabo con accento di sesta su parola sdrucciola: «Leggiamo qualche pagina, Francesca»

14 Ora Gavazzeni 2006

15 In particolare 13-106, 181-9, 190-216. Il libro raccoglie i saggi «Da d'Annunzio a Montale», uscito in Quaderno del Circolo filologico-linguistico padovano. Ricerche sulla lingua poetica contemporanea, 1966, e «D'Annunzio e la lingua poetica del Novecento», nel numero XL-XLI (1972) dei Quaderni dannunziani con gli atti del convegno sul tema. 
(Francesca da Rimini 3, 5, v. 924, corsivo aggiunto), già individuato da Mengaldo e ripreso da Bertazzoli. La tecnica, peculiare di d'Annunzio come poi di Montale, suscita vari effetti: di legame (cesura/ fusione) tra gli emistichi, perché, come scriveva Gavazzeni,

altera l'assetto con il sottrarre (una sorta di rubato) il tempo di una sillaba al secondo emistichio, per saldarlo in tal modo al primo (Gavazzeni 2006, 497)

e di marcatura semantica, in quanto lo sdrucciolo è di norma una parola-chiave in cui si concentra il significato del verso. Il modulo ha largo uso anche nella Francesca da Rimini (oltre 4.000 versi tra endecasillabi, settenari e quinari). Traggo qualche esempio dall'atto 5 , scena 4 :

PAOLO le stelle tramontavano nei tuoi capelli sparsi ai confini dell'ombra, ove labbia non giungono!

FRANCESCA Perdonami (vv. 281-283)

con le pupille aride e fisse, quale

tra le lance inflessibili quel giorno. (vv. 287-288)

le stelle non tramontano sul mare (v. 304)

et io non muoia senza aver divelta

dal tuo profondo

e assaporata l'infima radice

della mia gioia. (vv. 340-343)

Baciami gli occhi, baciami le tempie (v. 344)

La ricerca metrica influisce sui rimaneggiamenti del verso, naturalmente. Le varianti sono spesso dettate da un ritmo che non convince; tutto diventa invece definitivo quando 'si trova il ritmo':

ormai ho trovato il ritmo del libro; e mi basteranno sette o otto giorni di lavoro per compirlo

scrive d'Annunzio a Alberto Mondadori il 29 febbraio 1935, quando è al lavoro sul Libro segreto (Gibellini 1985, 185). Per restare in un caso già menzionato della Francesca da Rimini (i versi 411-412 dell'atto 2 , scena 3 ), due versi possono conoscere tre riformulazioni ravvicinate per una ricerca sonora:

e l'anima si sparge nella vostra 
voce e non so se soffra o se gioisca

o se voglia morire.

e l'anima mia trista mi si sparge

nel suono della vostra voce che è

sì strano.

e l'anima mia trista mi si sparge

nel suon di vostra voce che è sì strano.

Alla prima versione, poco convincente per varie ragioni (l'inarcatura forte tra possessivo e nome, il ritmo faticoso del secondo verso con accenti di prima, quarta e sesta), subentra un tentativo dalla struttura vocalica più felice nel primo verso $(a-i-a-i a-i-a-i-i-a)$ e con giochi allitteranti nei ravvicinati gruppi consonantici con $m$ e $s$ nel secondo (ima - mia - sta - mi - si - spa), che ancora risente però di una certa difficoltà (la successione faticosa degli accenti di seconda, quarta e sesta), risolta nell'ultima e definitiva versione, in cui la tonica forte cade su voce anziché su vostra, con un rafforzamento della parola semanticamente rilevante. Come conclude Bertazzoli, il ritmo (il suono, o, 'tommaseanamente', il numero) vale perché mette in contatto con «l'anima delle cose», che mi pare un bel modo di chiudere anche questo resoconto dello stato della filologia dannunziana.

\section{Bibliografia}

Antongini, Tommaso (1938). Vita segreta di Gabriele d'Annunzio. Milano: Mondadori.

Bàrberi Squarotti, Giorgio (1977). «I problemi dell'elaborazione del testo». Quaderni del Vittoriale, 1977, 13-23.

Beccaria, Gian Luigi (1975). L'autonomia del significante. Torino: Einaudi.

Bertazzoli, Raffaella (a cura di) (2004). D’Annunzio, Gabriele: La figlia di lorio. Gardone Riviera: Il Vittoriale degli Italiani.

Bertazzoli, Raffaella (2013). «Perizia metrica sul d'Annunzio tragico». Lombardi 2013, 29-43.

Bianchetti, Enrica; Forcella, Roberto (a cura di) (1965). D’Annunzio, Gabriele: Taccuini. Milano: Mondadori.

Bianchetti, Enrica (a cura di) (1976). D’Annunzio, Gabriele: Altri taccuini. Milano: Mondadori.

Caliaro, Ilvano (1995). «Fonti interne e fonti esterne». D’Annunzio, Gabriele. Il piacere. Introduzione di Pietro Gibellini. Milano: Garzanti, 421-52.

Campardo, Sara (a cura di) (2017). D’Annunzio, Gabriele: Elettra. Gardone Riviera: II Vittoriale degli Italiani.

Ciani, Ivanos (1975). Storia di un libro dannunziano: Le novelle della Pescara. Milano-Napoli: Ricciardi. 
Ciani, Ivanos (a cura di) (1976). D’Annunzio, Gabriele: "Il piacere" nella stesura preparata dall'autore per l'edizione francese del 1894. Con una introduzione di Ivanos Ciani. Milano: Il Saggiatore.

Contini, Gianfranco [1943] (1970). "Saggio di un commento alle correzioni del Petrarca volgare». Varianti e altra linguistica. Una raccolta di saggi (19381968). Torino: Einaudi, 5-31.

Contini, Gianfranco [1939] (1974). «Come lavorava l'Ariosto». Esercizî di lettura sopra autori contemporanei con un'appendice su testi non contemporanei. Torino: Einaudi, 232-41.

Croce, Benedetto [1947] (1948). «Illusione sulla genesi delle opere d'arte documentabile dagli scartafacci degli scrittori». Nuove pagine sparse. Napoli: Ricciardi, 190-1.

Crotti, Ilaria (2016). Lo scrittoio imaginifico. Volti e risvolti del d'Annunzio narratore. Avellino: Sinestesie.

De Michelis, Eurialo (1977). «Storia di un testo dannunziano. "Il compagno dagli occhi senza cigli"». Quaderni del Vittoriale 1977, 140-75.

Gavazzeni, Franco (1980). Sinopie di «Alcione». Milano-Napoli: Ricciardi.

Gavazzeni, Franco (1986). «Perizia metrica della Figlia di Iorio». Tiboni, Edoardo (a cura di), La figlia di lorio = Atti del VII Convegno internazionale di studi dannunziani (Pescara, 24-6 ottobre 1985). Pescara: Centro Nazionale di Studi Dannunziani, 57-101.

Gavazzeni, Franco (2006). «Perizia metrica della Figlia di lorio». Studi di critica e filologia italiana tra Otto e Novecento. Verona: Valdonega, 469-525.

Giallombardo, Federica Maria (2018). «Recensione a Pirovano, Donato (a cura di) (2018). D’Annunzio, Gabriele (2018). Francesca da Rimini. Roma: Salerno Editrice». Archivio d'Annunzio, 5, 2018, 191-4. URL https://doi.org/10.30687/ ada/2421-292x (2018-05-016).

Gibellini, Pietro (1975). «Per la cronologia di Alcione». Studi di filologia italia$n a, 33,398-424$.

Gibellini, Pietro (1976). «I pentimenti della Sera. Saggio di un commento alle correzioni di Alcyone». Mariano, Emilio (a cura di), D’Annunzio e il simbolismo europeo = Atti del convegno di studi (Gardone Riviera, 14-5-6 settembre 1973). Milano: Il Saggiatore, 343-67.

Gibellini, Pietro (1981). «Recensione a Gavazzeni 1980». Studi e problemi di critica testuale, 23, 203.

Gibellini, Pietro (1985). Logos e mythos. Studi su Gabriele D’Annunzio. Firenze: Leo S. Olschki Editore.

Gibellini, Pietro (1988). D’Annunzio, Gabriele: Alcyone. Milano: Mondadori.

Gibellini, Pietro (1995). D’Annunzio dal gesto al testo. Milano: Mursia.

Gibellini, Pietro (2008). «De Michelis e d'Annunzio». Bartolomeo, Beatrice (a cura di), Eurialo De Michelis (1904-1990). Pisa; Roma: Fabrizio Serra, 149-56.

Gibellini, Pietro (2013a). «D’Annunzio oggi e ieri: Bergamo, Pavia, Gardone». Lombardi 3013, X-XV.

Gibellini, Pietro (2013b). «Recensione a Gavazzeni, Franco (1980). Le sinopie di "Alcione". Milano-Napoli: Ricciardi». Lombardi 2013, 63-75 (riproduce Gibellini 1981).

Gibellini, Pietro (2014). «Critica genetica e variantistica continiana: esperienze di lavoro (Parini, Belli, Manzoni, D’Annunzio)». Ermeneutica letteraria, $10,115-25$. 
Gibellini, Pietro (a cura di) (2018). D’Annunzio, Gabriele: Alcyone. Commento di Giulia Belletti, Sara Campardo, Enrica Gambin. Scheda metrica di Gianfranca Lavezzi. Venezia: Marsilio.

Guglielminetti, Marziano (1977). «I 'taccuini' come libro della memoria». Quaderni del Vittoriale 1977, 176-203.

Isella, Dante (1972). «Dal taccuino inedito dell'Alcyone». Strumenti critici, 18, 163-73.

Lavezzi, Gianfranca (2018). «Nuove gocciole dal bosco dannunziano. Altre presenze della Pioggia nel pineto nel Novecento e oltre». Archivio d'Annunzio, 5, 11-26. URL https://doi.org/10.30687/ada/2421-292x/2018/05/०03.

Lombardi, Maddalena (a cura di) (2013). L'officina di d'Annunzio = Giornata di studi in ricordo di Franco Gavazzeni coordinata da Pietro Gibellini (Bergamo, 26 maggio 2012). Bergamo: Biblioteca civica "Angelo Mai".

Maiolini, Elena (a cura di) (2017). Tommaseo, Niccolò: Canti Greci. Parma: Fondazione Pietro Bembo; Ugo Guanda Editore.

Martignoni, Clelia (1977). «Sull'elaborazione delle Faville del maglio». Quaderni del Vittoriale, 308-53.

Martignoni, Clelia (2013). «Appunti sulla prosa». Lombardi 2013, 44-50.

Mengaldo, Pier Vincenzo (1975). La tradizione del Novecento. Milano: Feltrinelli. Monaci, Ernesto (1889). Crestomazia italiana dei primi secoli. Città di Castello: Lapi.

Montagnani, Cristina (a cura di) (2006). D’Annunzio, Gabriele: Maia. Gardone Riviera: Il Vittoriale degli Italiani.

Montagnani, Cristina (2013). «1903: un anno vissuto pericolosamente». Lombardi 2013, 20-8.

Montagnani, Cristina; De Lorenzo, Pierandrea (2018). Come lavorava d'Annunzio. Roma: Carocci.

Mussini, Gianni (2013). «Le lettere di Franco (un percorso tra filologia e amicizia)». Lombardi 2013, 51-9.

Pancrazi, Pietro [1939] (1944). «Nell'officina di Alcione». Studi sul D'Annunzio. Torino: Einaudi, 75-123. Roma: Treves-Treccani-Tumminelli.

Papponetti, Giuseppe; Cappellini, Milva Maria (a cura di) (2001). Ciani, Ivanos: Esercizî dannunziani. Prefazione di Pietro Gibellini. Pescara: Ediars.

Pirovano, Donato (a cura di) (2018). D’Annunzio, Gabriele (2018). Francesca da Rimini. Roma: Salerno Editrice.

Quaderni del Vittoriale (1977). «D’Annunzio, il testo e la sua elaborazione». Quaderni del Vittoriale, 5-6.

Sanjust, Maria Giovanna (a cura di) (2001). D’Annunzio, Gabriele: Elegie romane. Milano: Mondadori.

Scicchitano, Emanuela (2011). «lo, ultimo figlio degli Elleni». La grecità impura di Gabriele d'Annunzio. Pisa: Edizioni ETS.

Suitner, Franco (2012). «Su alcune 'resistenze' teoriche alla critica delle varianti». Ponti, Paola (a cura di), Letteratura e oltre. Studi in onore di Giorgio Baroni. Pisa; Roma: Fabrizio Serra, 522-6.

Tommaseo, Niccolò (1841-42). Canti popolari toscani corsi illirici greci. Venezia: Tasso.

Zava, Alberto (2016). «Rassegna di pubblicazioni per il centocinquantenario della nascita di Gabriele d'Annunzio". Archivio d'Annunzio, 3, 167-70. DOI http://doi.org/10.14277/2421-292X/AdA-3-16-12. 
Recensioni 



\section{Cristina Montagnani, Pierandrea De Lorenzo Come lavorava d'Annunzio}

\section{Elena Maiolini}

Università Ca' Foscari Venezia, Italia

Recensione di Montagnani, Cristina; De Lorenzo, Pierandrea (2018). Come lavorava d'Annunzio. Roma: Carocci, 141 pp.

Si è arricchita di un libro su Come lavorava d'Annunzio, scritto a quattro mani da Pierandrea De Lorenzo e Cristina Montagnani, la serie di 'Filologia d'autore' della collana «Bussole» di Carocci, diretta da Simone Albonico, Paola Italia e Giulia Raboni. In linea con gli intenti della serie, che intende offrire un orientamento pratico nello studio di grandi autori (il volume fa seguito a quelli dedicati a Gadda e Manzoni, rispettivamente di Italia e Raboni, e se ne attendono su Carducci e Boccaccio), offre l'inquadramento della poetica, la mappatura degli archivi dei manoscritti e dei libri e la presentazione della modalità compositiva, con un approfondimento su un'opera in particolare, in questo caso il ciclo poematico delle Laudi. A De Lorenzo si devono i primi quattro capitoli, mentre il quinto, sull'elaborazione del poema, è di Montagnani, già curatrice nel 2006 dell'edizione critica del primo libro del ciclo, Maia, per l'Edizione nazionale.

Il primo capitolo ( «Come lavorava: l' "intimo connubio dell'arte con la vita"», 7-32) prende le mosse dalla distanza che si interpone ormai da tempo tra la dimensione intima di uno scrittore raffinato, alla cui conoscenza la filologia d'autore offre una possibilità concreta, e il trionfalismo cui d'Annunzio stesso legò la propria immagine, alimentando un mito a cui poi non riuscì a sottrarsi, con un senso di angoscia che trapela bene da una pagina del Libro segreto proposta da De Lorenzo:

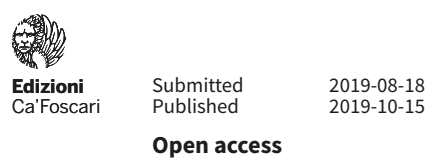

(C) 2019 | 어 Creative Commons Attribution 4.0 International Public License 
Questo ferale taedium vitae mi viene dalla necessità di sottrarmi al fastidio - che oggi è quasi l'orrore - d'essere stato e di essere Gabriele d'Annunzio, legato all'esistenza dell'uomo e dell'artista e dell'eroe Gabriele d'Annunzio, avvinto al passato e costretto al futuro di essa esistenza: a certe parole dette, a certe pagine incise, a certi atti dichiarati e compiuti: erotica heroica. (9)

Lattività letteraria è ripercorsa a partire dalle collaborazioni con la stampa giornalistica romana degli anni Ottanta, in cui d'Annunzio si rivela osservatore e interprete acuto di una cultura in evoluzione, oltre che scrittore fine e promotore abile di se stesso. Per queste ragioni meritano di non essere trascurate le cronache mondane, che l'autore stesso pensò di raccogliere includendone una selezione nell'Opera omnia progettata con Antonio Bruers. Meritano una lettura attenta anche per le consonanze con i racconti di San Pantaleone e, soprattutto ma non solo, Il Piacere: se pure vale il monito di Ivanos Ciani, che scoraggiava dal trovare nelle pagine di cronaca (ancora acerbe) avantesti importanti di quelle letterarie (molto più mature), non si può negare il valore della «ricchissima messe di immagini» (13) che esse contengono.

Si può fare un discorso simile per l'epistolario, da cui provengono ambientazioni che confluiscono nei romanzi, ricalibrate in un lavoro di cesellatura quasi sempre espansivo. In entrambi i casi il tema è molto interessante, perché conduce alla questione del rapporto tra arte e vita. Le lettere a Barbara Leoni, «già concepite - paradossalmente - come scene di una vita da reificare nelle pagine di un romanzo», migrano nel Trionfo della morte, di cui «costituiscono un vero e proprio materiale pregresso» (15). Così la «gamba nericcia, enorme come un tronco d'albero» di un uomo sofferente disteso davanti alla Chiesa dei Miracoli del santuario di Casalbordino, descritto in una lettera alla Leoni, diviene nel Trionfo

una gamba massiccia come un tronco di quercia, coperta di verruche e di croste gialle, qua e là abbronzita o nera, così smisurata che pareva non gli appartenesse (16)

dove notiamo il passaggio in seconda battuta dell'elemento cromatico nella coppia «abbronzita o nera», in cui la sfumatura diminutiva affidata al suffisso ( «nericcia») passa alla tonalità bronzea, prima che corvina, denotata dal participio; in primo piano sale invece l'elemento della dimensione («massiccia», da «enorme»), con la precisazione della specie arborea («albero» $\rightarrow$ «quercia»), un ampliamento comparativo («così smisurata che $[. .] »$.$) e l'aggiunta dei dettagli sulle lesioni cutanee.$

Di questa trasfigurazione letteraria del dato esistenziale Il fuoco costituisce, come è noto, un caso esemplare: sul suo altare creativo vengono sacrificati momenti privati della relazione con Eleonora Duse, ma anche dialoghi con Angelo Conti: «davvero la distanza tra arte e vita 
si assottiglia» e i profili biografici concorrono a definire i personaggi. Non si coglierebbe però il cuore della mimesi dannunziana limitandosi a considerare la vita come un cesto da cui estrarre spunti letterari: il rapporto tra arte e vita è più complesso, comprendendo un movimento in entrambe le direzioni in cui la letteratura restituisce «una realtà che la vita spesso non riesce a mostrare» (19). Come ha scritto Fausta Garavini (penso al saggio «'Io come io...'». Paragone. LXII, 9394-95, 2011, 36-55), in d'Annunzio la scrittura è un'operazione salvifica che afferma «la consistenza dell'io, tentando di ridurne a unità la pluralità intrinseca»: da questa prospettiva è inevitabile che la distinzione tra materia autobiografica e materia romanzesca si assottigli.

È quanto si constata lavorando sui Taccuini, pagine di servizio «dove registrare il momento dell'atto creativo colto sul nascere» (21). De Lorenzo propone alcuni casi (a due ma anche a tre colonne, per mostrare la migrazione della nota diaristica in più di un'opera letteraria) che esemplificano le varie forme che può assumere l'appunto di «preistoria testuale», con l'espressione coniata da Pietro Gibellini, che per primo prese in esame in modo nuovo e funzionale alla comprensione della storia dei testi i quadernetti con guizzi lirici, note paesistiche, descrizioni da sviluppare che già contengono gli elementi grafici, fonetici, sintattici, metaforici propri della scrittura dannunziana. ${ }^{1} \mathrm{Il}$ modus operandi dello scrittore si realizza in un movimento di trascendenza che lo accomuna al Francesco Paolo Michetti fotografo della vita abruzzese: a partire da un'impressione sensoriale si supera il puro descrittivismo svelando, «nella potenza espressiva delle immagini, una forza primigenia» (26), una dimensione mitica e atemporale in cui si avverte il dialogo con altri grandi artisti del Simbolismo europeo.

Il secondo e il terzo capitolo («L'autore e le sue carte», 33-45; «In biblioteca», 47-58) risultano particolarmente utili per chi voglia un panorama complessivo sulla dislocazione dei manoscritti e dei libri. De Lorenzo vi traccia le vicende degli autografi, circolati già in vita grazie all'abilità di d'Annunzio di promuovere se stesso (immagine e testi), monetizzando sul commercio dei manoscritti predisposti appositamente per raggiungere il mercato degli appassionati soddisfandone il «vezzo salottiero» (34) e finanziare così nuove opere, in un circolo auto-rigenerativo. Una ricostruzione sintetica e pratica dell'assetto delle carte descrive gli archivi del Vittoriale, costituiti con l'Atto di donazione allo Stato pubblicato sulla Gazzetta Ufficiale del Regno d'Italia il 4 dicembre 1930 (34-6), quindi attraverso l'impegno di Antonio Bruers prima e di Emilio Mariano poi (36-40); le importanti raccolte nei fondi della Biblioteca Nazionale Centrale di Roma, formate grazie al lavoro di Annibale Tenneroni e di Federico Gentili di Giuseppe e dei suoi eredi, che crearono delle collezioni arricchite ulterior-

Cf. in questo stesso numero, articolo dell'Autore, § 4 «Preistoria testuale». 
mente da acquisti compiuti nella seconda metà del Novecento, oggi facilmente consultabili grazie a un progetto di archiviazione elettronica (41-4); infine i fondi - più scarni, ma sempre preziosi - dell'Istituto per il Teatro e il Melodramma della Fondazione Giorgio Cini di Venezia, con i copioni autografi delle tragedie di mano della Duse (44-5).

Alla biblioteca di un grande feticista del libro è dedicato il terzo capitolo. «Ricostruirla porta necessariamente a ripercorrere l'intera vita di d'Annunzio» (47): il periodo romano a cui risalgono i primi acquisti (la biblioteca itinerante che seguirà il proprietario a Napoli e a Francavilla); l'epoca della Capponcina, dove nel marzo 1898 approda il nucleo più consistente (oltre 4.000 volumi), sistemato e arricchito con l'aiuto di Tenneroni e di Conti (raddoppia in meno di dieci anni); l'esilio francese, con le vicende intercorse negli anni del dissesto economico, quando d'Annunzio cerca di arginare la perdita dei libri che a Settignano finiscono sotto sequestro o vanno all'asta affidandone la custodia a amici fidati, e gli arricchimenti della 'Bibliothecula gallica', otto vagoni di volumi traslocati in Italia nel 1919 e confluiti nella 'Colonica' di Gardone dopo l'esperienza fiumana. Infine Cargnacco, dove i libri di d'Annunzio si aggiungono a quelli già appartenuti a Henry Thode, per raggiungere circa 33.000 volumi, dislocati nelle varie stanze della villa grazie all'impegno del propositivo e accurato Bruers. La seconda parte del capitolo orienta in tale mole poderosa indicando quanto fu senz'altro oggetto di un'attenzione diretta da parte di d'Annunzio: dizionari, repertori, carteggi, antologie, opere narrative, la cui lettura assidua è testimoniata da note a margine, piegature agli angoli, cartigli e foglie e fiori essiccati tra le pagine.

Il libro accompagna quindi nel retrobottega dell'officina dannunziana offrendo nel corposo e denso quarto capitolo (59-83) un panorama generale di quanto si intuisce sbirciando d'Annunzio «sulla scrivania», come annuncia il titolo della sezione e pure mostra una fotografia dello scrittore al tavolo di lavoro, nel 1921 (62). Alla classificazione delle tipologie di carte autografe (le belle copie destinate a diventare dei doni - il codice calligrafico delle Laudes creaturarum predisposto per la Duse - o dei cimeli per gli estimatori, sostanzialmente estranei alla storia del testo; le copie per il tipografo; le minute e gli appunti preliminari), seguono dei paragrafi dedicati al tipo di studio che tali materiali richiedono: il filologo deve ricostruirne la datazione, spesso trascurata, occultata o persino manomessa da d'Annunzio, privo di una visione storicista non solo per disinteresse, ma anche per una «vera strategia: storicizzare, forse, è un atto banalizzante» (61).

Affrontando quindi la questione delle varianti appoggiandosi ai casi dei manoscritti della Leda senza cigno, delle Vergini delle rocce, del Ferro e poi soprattutto del Fuoco, De Lorenzo descrive il peculiare metodo di lavoro che genera carte sciolte «molto travagliate, sofferte, pasticciate» su cui il poeta «prova e riprova» sintagmi, rime inceppate, giri di parole, prima di trovare la versione convincente e gettare 
quindi nel cestino quanto testimonia un travaglio che desidera tacere, volendo dar credito all'immagine di sé come di uno scrittore che compone di getto, sotto l'impulso «di un'ispirazione superiore» (63). Alcuni cambiamenti si risolvono invece in modo immediato sulla stessa carta, in una scrittura che consuma all'istante ogni dubbio e ogni mutamento, senza ripensamenti radicali della poetica (alla Manzoni). De Lorenzo spiega le direzioni costanti che si intuiscono nel correggere: «dilatazione anzitutto, e al contempo un innalzamento del registro linguistico» verso una maggiore puntualità (64-5).

La tendenza a impreziosire il dettato all'interno di una ricerca di equilibrio si nota molto bene negli esempi proposti all'interno del § 4.4, dedicato ai manoscritti del Fuoco (66-72), un caso di studio che permette di «affinare le tecniche di indagine più adatte ai testi dannunziani» (67). L'osservazione dei mutamenti dell'onomastica e della grafia, del materiale documentario extratestuale (lettere, appunti e taccuini), della misura della carta Fabriano, insieme alle informazioni che si ricavano dagli epistolari e dalla versione francese del romanzo sono elementi che aiutano a datare le varie fasi del testo. De Lorenzo chiarisce la metodologia propria degli studi variantistici richiamando la distinzione teorica definita da Gibellini tra preistoria e protostoria testuale e tra micro e macrovarianti: alcune lettere mandate a Hérelle in occasione dell'uscita del Fuoco sono un esempio proficuo della modalità di revisione, quasi sempre contemporanea al primo getto e solo raramente successiva alla stampa, funzionale a approntare una nuova edizione strutturalmente differente di un'opera già pubblicata: alla storia dei tre soli casi del genere - il Canto novo, l'Intermezzo di rime e l'Isaotta Guttadàuro - è dedicato un paragrafo apposito. Chiude il capitolo che indaga il modus operandi la storia dell'Edizione nazionale, con la costituzione dell'Istituto nel 1926, le prime pubblicazioni curate redazionalmente da Angelo Sodini, quelle più economiche dell'Oleandro (1931-37), il nuovo corso negli anni Ottanta e quindi la ripresa recente, di cui l'ultimo frutto è l'edizione critica dell'Elettra a cura di Sara Campardo (2017).

Il libro termina con un affondo su «Un caso di 'studio': l'elaborazione delle "Laudi"», nel quinto capitolo (85-133), curato da Montagnani. La studiosa, forte dell'esperienza sul primo libro del ciclo, ricostruisce le tappe della sua realizzazione dall'exploit del 1899 allo stallo dovuto all'avanzare di altri lavori (il Fuoco, la Francesca da Rimini, la revisione delle novelle giovanili), ma anche alla difficoltà di incanalare i testi del canzoniere in una forma convincente, il momento «carsico» (89) del fluire poetico precedente alla soluzione cui approda nel 1903. La rievocazione poggia sulle acquisizioni procurate dai lavori di Gibellini usciti a ridosso dell'edizione critica di Alcyone (1988), coi quali lo studioso, occupandosi delle varianti di struttura e stabilendone la datazione, prima ignota o mal nota, giunse a spiegare perché d'Annunzio potesse chiamare "poema» la sua raccolta di liriche, dalla struttura finale piuttosto forte, aggiustata in corso d'opera. 
Montagnani si sofferma su alcuni snodi importanti di questo travaglio, offrendo la trascrizione di alcuni manoscritti fondamentali per la ricostruzione della cronologia del ciclo poematico, facendo notare, negli elenchi di titoli, cassature e inserimenti: nei due lati di un autografo custodito negli archivi del Vittoriale, riprodotto all'interno del capitolo (92-3, figg. 3a-b), si osserva il ribollire delle idee e la fatica evidente di incanalarle, nella ricerca di una forma che «conta più di ciò che va a racchiudere», con «una dinamica che più dannunziana non si può» (91). Le debolezze strutturali si risolvono un po' per volta, come testimoniano gli elenchi di titoli trascritti nel capitolo, che guida all'osservazione degli appunti in cui si può intercettare «la volontà di struttura» (97) e cogliere gli indicatori di svolta nella sua definizione, come il termine post quem della citazione interna a alcuni versi della Laus, evidentemente successiva alla lettura del Voyage in Grèce di Jean Moréas, pubblicato nel 1902 (cf. 100), ma anche l'indice del comunicato editoriale Treves comparso sull'Illustrazione italiana nel gennaio 1903 (riprodotto nella fig. 4, 102). Montagnani approfitta di questi e di altri segnali per mostrare come al progetto d'Annunzio arrivi gradualmente, risolvendo difetti e mancanze libro per libro fino alla svolta dell'immissione delle idee nietzschiane sulla mitologia e del congedo dalla terra ellenica; e guida il lettore all'osservazione di una concomitante mutazione nella tecnica compositiva: «canti stesi in fretta, in pochi mesi se non in pochi giorni» (107), sotto la guida del ritmo-significante più che della parola-significato.

Gli ultimi paragrafi affrontano la concretezza di alcune carte degli autografi di Maia e Alcyone, di cui si trascrivono alcuni versi con i relativi apparati delle varianti delle edizioni critiche curate, rispettivamente, dalla stessa Montagnani nel 2006 e da Gibellini nel 1988 - quando il libro va in stampa presso Carocci non è ancora stato pubblicato, infatti, l'aggiornamento dell'edizione critica uscito in coedizione tra l'Edizione nazionale e i Classici Marsilio (2018): Montagnani può annunciarne l'uscita imminente (126), ma non riprodurne la nuova rappresentazione tipografica delle varianti aderente alla successione cronologica, già definita con l'Elettra di Campardo (per cui cf., in questo numero, «Stato, problemi, applicazioni critiche della filologia dannunziana», § 4 «Criterio topografico o criterio cronologico») -. La riproduzione della versione manoscritta di alcune strofe e di frammenti preparatori (figg. 5 e 6a-b, 118-20) permette di osservare l'affiorare di un'idea, di qualche «immagine ancora priva di struttura» (121), di suggestioni provenienti dalla lettura di altri testi e di altre «schegge testuali» (124) che trovano poi un'elaborazione nelle varie stesure dei brani, ma anche di rilevare i momenti di crisi compositiva, gli inceppamenti del «miracoloso» (133) fluire dei versi dalla penna dell’Immaginifico, segnalati da spazi bianchi, parole cassate e lezioni rimaste sospese: tracce affidate talvolta a foglietti malconci sfuggiti alla volontà dell'autore e arrivati nelle mani «di quella pericolosa specie di studiosi che praticano la filologia» (133). 


\section{Cecilia Gibellini La miglior parte della mia anima. Lettere alla moglie (1883-1893)}

Paolo Parachini

Università Ca' Foscari Venezia, Italia

Recensione di Gibellini, Cecilia (a cura di) (2018). D’Annunzio, Gabriele: La miglior parte della mia anima. Lettere alla moglie (1883-1893). Milano: Archinto, 274 pp.

Siamo nel 1883, il giovane e ambizioso Gabriele d'Annunzio (18631938) dalla natia Pescara si è trasferito a Roma, smanioso di tentare la scalata nell'alta società. Avvicina pertanto la bellissima Natalia Hardouin di Gallese Altemps (moglie del duca Jules), appassionata di arte e poesia, nonché ammiratrice del giovane poeta che incanta i salotti mondani di Roma. Ma ben presto d'Annunzio rivolge la sua attenzione alla figlia diciottenne Maria, alla quale scrive lettere appassionate con versi che avrebbero intenerito persino un cuore di ghiaccio: «Ancora io sento su la mia bocca | fremer le tue labbra voraci... | Oh se il mio lungo dolor ti tocca, | vieni a' miei baci!». Il duca, padre di Maria nonché militare di origine francese, si oppone però fermamente a qualsiasi relazione fra la figlia e il giovane poeta che giudica un parvenu. Ma l'ambizioso Gabriele non demorde e inscena un'autentica fuitina con la giovane Maria. Ė una fuga d'amore, messa in opera con la sortita di Maria dal palazzo Altemps all'alba del 28 giugno 1883 e la corsa in treno da Roma a Firenze, dove tuttavia la coppia trova a attenderla le autorità, giunte a recuperare la giovane per farla riaccompagnare a Roma. Si suppone che a orchestrare l'inseguimento sia stato lo stesso Gabriele, interessato a sollecitare la scoperta che avrebbe fatto scandalo e reso inevitabile il matrimonio, cui infine il duca dovette piegarsi, vittima della scaltra congiura. Quando il matrimonio tra i

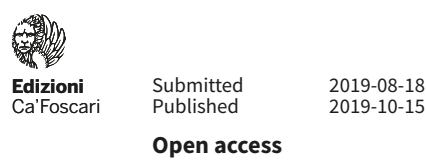

(C) 2019 | 어 Creative Commons Attribution 4.0 International Public License 
due giovani si rese inevitabile, il duca Jules troncò ogni rapporto con la moglie, la figlia e il genero, vanificando le ambizioni di quest'ultimo: cosicché d'Annunzio, lungi dal potersi fregiare del titolo di 'Principe romano' come il suo eroe Andrea Sperelli, dovrà contentarsi di quello autoironico di «Duca minimo», con cui firmerà a partire dal 1885, le sue cronache mondane sulla Tribuna. Sembra la trama di un film, escogitato da un maestro della comunicazione, che finora i biografi hanno narrato senza poter distinguere chiaramente mito e realtà. Solo ora, attraverso le lettere inedite - pervenute recentemente alla Biblioteca Nazionale di Roma - diventa possibile ricostruire, almeno per lunghi tratti, la duratura e complessa relazione che legò d'Annunzio alla moglie: tale infatti Maria rimase, dal momento che le pratiche per il divorzio da lei avviate nel 1904, quando da anni viveva separata da Gabriele, furono interrotte su richiesta del poeta, non contento di aver cominciato presto a tradirla, lasciandola con tre figli dopo aver sperperato l'inimmaginabile. Il divorzio, allora possibile in Svizzera, sarebbe bastato al padre di Maria per riammetterla all'eredità: ma il poeta antepose la propria immagine, che sarebbe stata scalfita, ai doveri di coniuge e padre. Con commovente generosità, Maria non ebbe, né allora né poi, parole men che signorili nei confronti dello scrittore.

Questo volume raccoglie le lettere che Gabriele d'Annunzio inviò alla moglie Maria Hardouin nei primi dieci anni del loro tumultuoso matrimonio, anni fondamentali per la crescita dello scrittore. Pubblicate qui per la prima volta, per la cura di Cecilia Gibellini, esse compongono il nucleo di maggiore interesse, biografico e letterario, all'interno di un carteggio che conta complessivamente diverse centinaia di messaggi, tra lettere, biglietti e telegrammi, che si snoda per più di mezzo secolo: dalle prime settimane di conoscenza tra i due (inizio 1883), fino agli ultimi mesi di vita del poeta, quando Maria passava lunghi periodi nella villa Mirabella a lei riservata al Vittoriale, dove del resto la 'principessa di Montenevoso' fu sepolta alla sua morte, avvenuta nel 1954, sedici anni dopo quella del marito.

«Quanto tempo è passato, e come rapido! [...] E quanti avvenimenti, e non tutti lieti! E tu quanti dolori hai avuti da me, povera Maria! Ma tu sei molto buona e molto paziente; ed io trovo in te, nei momenti più difficili e più tumultuosi, sempre un cuore amico e un intelletto pronto. Mi è dolce oggi dirti queste cose. Troppe volte io sono ingiusto con te duro e violento e incurante; ma conservo sempre nel profondo dell'anima una grande e calda ammirazione per te e una tenerezza non mutabile» (lettera del 28 giugno 1887).

Gabriele ha l'arte di lasciarsi perdonare, e le lettere, se gettano ombre sinistre sull'uomo, pronto a dirsi sull'orlo del suicidio e causa invece del suicidio effettivamente tentato da Maria precipitatasi dal balcone per disperazione, contengono pagine di qualità ammirevole: descri- 
zioni paesistiche, momenti di tenerezza verso i bimbi coloriti dall'emergere di dialettalismi abruzzesi, sfoghi passionali e confessioni di sconforto, squarci illuminanti sul suo lavoro creativo.

La curatrice, Cecilia Gibellini, ha provveduto a datare con precisione le lettere, metà delle quali erano prive di collocazione temporale (operazione purtroppo non condotta nell'edizione del carteggio d'Annunzio-Duse uscita nel 2010 a cura di Franca Minnucci), a corredarle di una puntuale annotazione e a premettere al volume un saggio acuto e penetrante che delinea l'intera parabola della relazione tra i due protagonisti, dall'origine fino alla morte. La curatrice ha potuto mettere a profitto le sue competenze pregresse di filologa, di epistolografa e di dannunzista: basti richiamare, per le prime, l'edizione delle novelle inedite e disperse di Giambattista Casti e la ricostruzione della incompiuta monografia su Petrarca di Mario Pomilio; per le seconde la cura dei carteggi Scheiwiller-Pizzuto, Saba-Sereni e Bianconi-Beretta; per le terze gli studi su d'Annunzio, specie per il rapporto con le arti figurative.

Ora, la pubblicazione dell'epistolario completo di d'Annunzio costituisce un'impresa difficilmente realizzabile; e per dare un'idea del ventaglio tematico e espressivo Marziano Guglielminetti e Elena Ledda scelsero opportunamente la soluzione antologica, raccogliendo nel 2004 Il fiore delle lettere dannunziane. Anche questo volume ha scelto una via selettiva, pubblicando seppur integralmente la prima e più interessante sezione dell'ampio carteggio: in attesa di un auspicato completamento, possiamo ben dire tuttavia che esso colma la maggior lacuna nella corrispondenza del vate con le donne più rilevanti della sua vita: Lalla Zucconi, Barbara Leoni, Giuseppina Mancini, Eleonora Duse, Olga Levi, Luisa Baccara, aggiungendo nuovi documenti per i biografi e nuove prose d'arte per i critici. 



\section{Maurizio Serra D’Annunzio le Magnifique}

Veronica Tabaglio

Università Ca’ Foscari Venezia, Italia

Recensione di Serra, Maurizio (2018). D’Annunzio le Magnifique. Paris: Éditions Grasset et Fasquelle, 704 pp.

Ultimo tassello (cronologicamente parlando) del mosaico sul Novecento che Maurizio Serra va componendo da diversi anni, D'Annunzio le Magnifique intende confrontarsi con una figura che di quel secolo è stata indubbia protagonista. È un compito che Serra svolge ben consapevole dei rischi e delle difficoltà che attendono chi si accinga a stendere una biografia di Gabriele d'Annunzio a tutto tondo. D’altra parte, non è la prima volta che l'autore si cimenta con questo genere: sempre per i tipi di Grasset, sono uscite, rispettivamente nel 2011 e nel 2013, le biografie di Curzio Malaparte e di Italo Svevo: Malaparte, vies et légendes (omaggiata quello stesso anno del Prix Casanova e del Prix Goncourt de la Biographie) e Italo Svevo ou l'antivie. Conscio dunque delle specificità di questo genere e della mole di materiale a sua disposizione, Serra affronta nell'Introduzione le due questioni che motivano il suo lavoro: l'esigenza di un nuovo scritto sulla vita di d'Annunzio e il taglio con cui affrontarlo. Nonostante la distanza temporale, infatti, l'autore ritiene che la figura di questo autore sia ancora gravata dal peso di notevoli semplificazioni critiche, che ne ostacolano una vera conoscenza e un più fondato apprezzamento; a questa condizione diffusa si aggiunge il particolare caso del pubblico francese, presso cui forse non è nemmeno così nota l'immagine stereotipata.

Inevitabilmente, è necessario - nel caso di d'Annunzio ancor più che con altri autori - porsi la domanda riguardo alla possibilità di

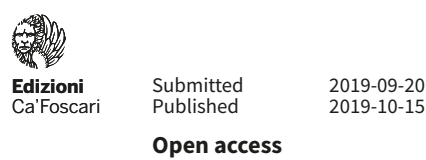

(c) 2019 | () Creative Commons Attribution 4.0 International Public License

Citation Tabaglio, Veronica (2019). Review of D'Annunzio, le Magnifique, by Serra, Maurizio. Archivio d'Annunzio, 6, 141 -146. 
tracciare una linea fra l'uomo e il personaggio che egli decise di interpretare, ben sapendo che «Le personnage occulte l'homme du début à la fin, sans la moindre fissure» (12) e che dunque ogni documento, ogni aneddoto (che sia raccontato dal Vate, dal suo factotum Antongini o da chiunque altro) deve essere vagliato e soppesato con cura. Serra avverte il lettore, conseguentemente, dell'intenzione di non soffermarsi su ogni episodio od ogni aspetto con la stessa attenzione: in parte per l'impossibilità di un simile compito, o - a voler essere più precisi - per la sua inservibilità: anche ambendo a riunire in un'unica opera un insieme così vasto di dati e interpretazioni, di che utilità potrebbe essere al pubblico, soprattutto quando esso non sia specializzato, se non venisse proposto anche un percorso di lettura?

Così come aveva fatto nelle precedenti biografie, dunque, l'autore esplicita il taglio che ha inteso dare all'opera, rimarcando però una notevole differenza: se con Svevo e Pirandello, Serra aveva voluto indagare gli elementi costitutivi della loro modernità, da un lato osservando lo sviluppo del romanzo contemporaneo attraverso l'ironia e il mimetismo, e dall'altro seguendo lo stravolgimento delle convenzioni teatrali e l'utilizzo del cerebralismo come strumento per attaccare la realtà, con d'Annunzio l'individuazione è meno immediata. Uno degli obiettivi dichiarati, dunque, è anche uno dei più ambiziosi che il biografo possa porsi: indagare la figura di d'Annunzio a tutto tondo, come autore e come uomo inserito in un preciso contesto, in modo tale da consentire al lettore (in particolare, al lettore francese) di formarsi un proprio giudizio su quanta parte di essa può essere ormai considerata anacronistica e quanta, invece, conservi tuttora una sua carica vitale. Da questo punto di vista, uno dei meriti dell'opera di Serra riguarda il tentativo di inserire d'Annunzio in una temperie culturale che oltrepassi i consueti confini della letteratura e della cultura nazionali, sottolineandone invece le relazioni, le affinità e i contrasti con alcuni dei maggiori scrittori del Novecento - fermo restando il carattere non specialistico dell'opera. Senza dunque ambire a un discorso critico compiuto a proposito della scrittura e dello stile dannunziani, Serra ne rileva alcune caratteristiche che a suo parere, lungi dal mostrarne la datata artificiosità, li inquadrano al contrario in una cornice europea e globale di tutto rispetto, evocando tra i tanti i nomi di Joyce, Faulkner e Borges:

Certes, l'avalanche de citations classiques, de formules recherchées, de références érudites à faits et gestes de l'Antiquité, du Moyen Âge, de la Renaissance, etc., engorge périlleusement son style. Mais que dire du côté bric-à-brac d'Ulysse ou de Finnegans Wake ? Des obscurités et des anacoluthes de Faulkner, Dos Passos ou Beckett? Des mythomanies de Heidegger et des pastiches de Sartre ? Des complaisances, parfois erronées, de Shaw, Wells, Huxley, Gombrowicz ou Borges? Tout auteur, tout grand auteur, 
a son langage, y compris ses bredouillements, qu'il faut savoir entendre et respecter. (30)

Altrettanto si spende Serra per rimarcarne la fortuna d'oltralpe, sottolineando - sulla falsariga del celeberrimo giudizio montaliano - la sua imprescindibilità, sia che lo si ammiri sia che lo si disprezzi:

D’Anatole France à Remy de Gourmont, de Barrès à Léon Daudet et Maurras, de Jules Renard à Romain Rolland et Julien Benda, de Gide à Valéry, de Valery Larbaud à Charles Du Bos et Jules Romains, de Jouve à Cocteau et André Maurois, sans oublier au passage Proust, aucun de ses contemporains n'a pu l'ignorer. Mais très peu l'ont compris. Au lendemain de sa mort, Claudel n'hésitait pas à le comparer à Jean Lorrain, « dont les pièces et les romans du signor d'Annunzio forment la suite bouffonne ». [...] La génération « $1900 »$ l'a également lu, avec agacement ou admiration : Drieu La Rochelle, Aragon, Benoist-Méchin, Marguerite Yourcenar... Malraux, qui à vingt ans jurait à Clara que « je finirai bien par être D'Annunzio», a fouillé dans Nocturne et Le Livre secret avec la même énergie qu'il employa à piller les temples khmers, et il en a imité faits et gestes, avant d'oublier son nom. (34)

Legandosi a questo obiettivo o criterio guida per la sua opera, Serra motiva la scelta di non dedicare lo stesso spazio a ogni aspetto della biografia, specificando come alcuni argomenti siano già stati abbondantemente affrontati (come la vita amorosa), mentre ad altri non sia ancora stato dedicato il giusto approfondimento. Nonostante questo, non poche pagine sono dedicate alle diverse compagne e amanti di d'Annunzio, pure già abbondantemente (e a volte morbosamente) raccontata da altri autori; sarebbe stato arduo compito, d'altro canto, voler ripercorrere le tappe fondamentali di un percorso così segnato dall'avvicendarsi di figure femminili senza dargli il necessario rilievo.

Il vero fulcro, tuttavia, che l'autore si propone di esaminare più da vicino, è sul ruolo pubblico di d'Annunzio, anche e soprattutto nei casi in cui tale ruolo dev'essere ricondotto a una responsabilità storica. Il percorso di ricostruzione di Serra è sempre estremamente certosino, fin dai primi anni di vita del Pescarese, ma com'è comprensibile, date le premesse, questo si fa ancora più accurato quando si trova a ripercorrere i periodi di Fiume e del Vittoriale, con una particolare attenzione a quest'ultimo. Per quanto concerne l'occupazione fiumana, Serra non pretende di arrivare a comporre un quadro esaustivo di un caso tanto complesso; gli preme di illustrare come essa sia inscindibile dall'intera concezione della guerra di d'Annunzio (il celeberrimo «J'adore la guerre!») e soprattutto del ruolo di poeta-vate, inteso in senso dantesco - che rende dunque pienamente conto del suo diretto attivismo militare: 
Mais s'ils [Daudet, Barrès, Kipling, Mann] s'engagent à fond par la plume et par l'exemple, on les trouve rarement en première ligne, surtout à l'âge du volontaire D’Annunzio. "Ce dur petit soldat», comme l'appellera Barrès [...], sera combattant autant que poète et orateur, du début à la fin du conflit, au point de mériter quatre avancements, jusqu'au grade de général de brigade aérienne à titre honoraire. [...] Si on ajoute à cela une médaille d'or, cinq d'argent, une de bronze et plusieurs autres décorations, dont la croix de guerre française et la Military Cross britannique, on a le droit de se demander qui en a fait autant dans la communauté littéraire de la Grande Guerre, et non seulement. (365)

Un attivismo che quindi non solo preesiste al fascismo, ma che a esso si affianca soltanto in parte e solo per un certo periodo; la distanza di fondo, nella lettura di Serra, poteva d'altra parte essere già intuita proprio grazie ad alcuni caratteri dell'esperienza di Fiume: non a caso, sottolinea, fu il primo 'Stato' a riconoscere la futura URSS, guadagnandosi così l'attenzione dello stesso Lenin.

Anche in virtù dell'enorme risonanza internazionale dell'impresa fiumana e del ruolo che d'Annunzio assume nei primi anni del fascismo, Serra si concentra allora sulla fase crepuscolare della vita di d'Annunzio, fase che a suo parere è stata finora trattata con imbarazzo o con scherno, in entrambi i casi senza la dovuta profondità, e si interroga su quanto possa essere corretto il giudizio di chi ritiene abbia fatto la sua parte e sia già escluso dai giochi: «Joyce, en écrivant à Yeats, dira également que D’Annunzio était fini, mais qu' il fut pourtant à une époque un merveilleux poète'» (543). Per quanto indubbiamente meno frenetica e meno grandiosa che in passato, la vita di d'Annunzio non cambia di segno in pochi mesi. Serra indaga dunque con scrupolo l'evoluzione dell'uomo-politico: le visite di Gramsci e di Čičerin (commissario del popolo agli Esteri della Russia sovietica), l'avvicinamento e poi l'appoggio al fascismo, cui segue presto un progressivo distacco. Già nel 1923 infatti Mussolini gli suggerisce freddamente di occuparsi solo di poesia: un consiglio che, superata la delusione, lo porterà a concentrarsi nuovamente sulle sue opere, pianificandone l'edizione nazionale e fondando un istituto per la rappresentazione dei suoi drammi. Si rivela così un intento riguardante più la conservazione che la creazione ex novo; la caduta in disgrazia, per quanto smorzata, finisce per riflettersi sull'uomo-artista:

D’Annunzio, qui avait toujours su captiver son public d'une manière ou d'une autre, se rendit compte du coup que les jeunes tournaient le dos à son œuvre, comme ils abandonnaient les drapeaux légionnaires. Il disposait encore du socle des classes moyennes, qui achetaient ses livres et assistaient à ses représentations ; mais elles étaient plus attirées par le frisson peccamineux du d'annun- 
zianisme que par ses réelles qualités d'auteur. Le personnage qui avait nourri l'écrivain commençait à lui nuire, et la tendance ne s'est plus arrêtée jusqu'à nos jours.

Da questa constatazione Serra prosegue nel ripercorrere la curva discendente della parabola dannunziana, dalla posizione di frondista interno alle complicazioni famigliari fino alla «turpe, l'oscena vecchiezza», di cui il Libro segreto è perfetta emanazione e incarna il corrispettivo opposto del Piacere della sua giovinezza.

Il percorso che propone Serra, dunque, segue fedelmente la scansione cronologica degli eventi, senza per questo rinunciare a un taglio tematico che gli consente interessanti collegamenti e anticipazioni o riprese. L'opera si articola perciò in quattro parti, ognuna delle quali è tripartita e ha per titolo un appellativo eloquente: la prima, L'innocent, copre i primi trentatré anni di d'Annunzio seguendone la formazione e gli spostamenti tra Pescara, Roma, Napoli, Venezia e la Grecia. Nel primo capitolo, «Le démon de la dualité», viene presa in esame una scissione intrinseca alla natura di d'Annunzio, su cui poi Serra tornerà a più riprese nelle successive sezioni: una tensione mai pienamente risolta tra preziosismo e richiamo alla terra primigenia, tra l'esaltazione delle virtù e l'inseguimento delle pulsioni, tra d'Annunzio e Rapagnetta. La seconda parte, intitolata Le conquérant, si concentra sugli anni compresi tra il 1897 e il giugno 1914, dal suo impegno come deputato al periodo fiorentino, per arrivare poi al trasferimento in Francia e ai suoi rapporti con i maggiori nomi della cultura e della vita mondana di Parigi. La terza e la quarta sezione, come già in parte accennato, si incentrano invece sul periodo della Grande Guerra (in particolar modo di Fiume) e del Vittoriale: Le commandant copre dunque gli anni a partire dal luglio 1914, con la visita al fronte della Marna e l'arruolamento volontario, fino al cosiddetto Natale di sangue del 1920; L'agonisant ripercorre i restanti diciassette anni di vita di d'Annunzio, dalla decisione di fissare la proprio dimora sul lago di Garda al suo progressivo isolamento nella gabbia dorata del Vittoriale, passando attraverso il graduale distacco da Roma e da Mussolini, cui sconsiglia inutilmente l'alleanza con la Germania.

A queste parti fanno seguito alcuni strumenti per il lettore: una tavola cronologica, per ricostruire rapidamente i principali snodi temporali della biografia dannunziana, e una bibliografia delle opere (senza alcuna pretesa di esaustività). Va ricordato anche il breve epilogo, intitolato «Oh! Souvenirs, doux et déchirants! ...», in cui l'autore ribadisce la necessità di confrontarsi con la figura di d'Annunzio armati di vera onestà intellettuale per poter tentare un giudizio meno parziale: un giudizio che riconosca, ad esempio, come uno dei maggiori limiti dell'opera dannunziana sia l'assenza di due temi cardine della modernità, ovvero la memoria (ricordiamo la sua insoffe- 
renza per Proust) e il confronto con il male. Ma gli si dovrà altresì riconoscere una determinazione inflessibile nell'aver perseguito, per tutta la vita, un ideale di poesia come atto combattivo ineludibile, in grado di superare ogni barriera di genere e di tempo. Così si chiude allora la paziente ricostruzione di Serra, lungo tutte le cui pagine risuona l'augurio iniziale:

Si on ajoute que c'est surtout un grand, souvent un très grand écrivain, le moment n'est-il pas venu de relire sans préjugés ? Ce livre est donc dédié au public français s'aujourd'hui : non pas pour changer son jugement sur l'homme et l'œuvre, mais afin qu'il puisse s'en former un. Et situer à la place qui est, qui restera la sienne, ce D’Annunzio, malgré tout, magnifique. 

Rivista annuale

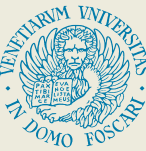

Università

Ca'Foscari

Venezia

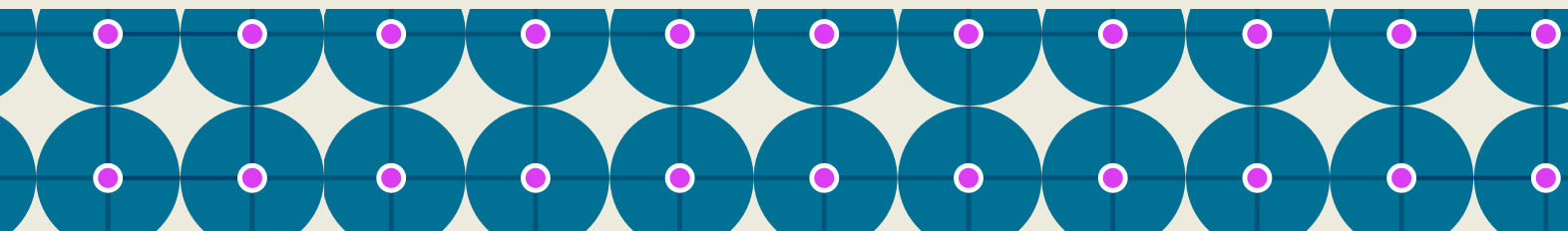

0 o 0 o 0 o 0

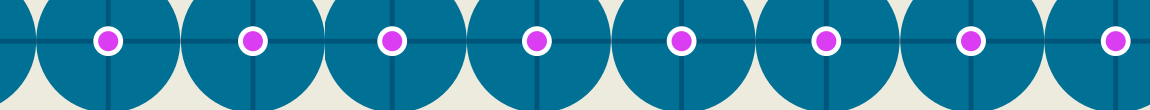

0 o 0 o 0 o 0

$\gamma$

0

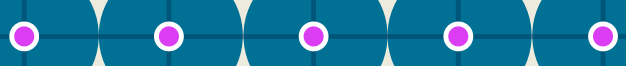

10

0

0

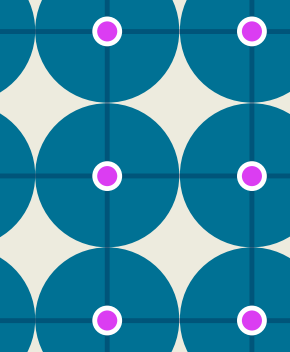

1000

$\begin{array}{lllllll}0 & 0 & 0 & 0 & 0 & 0\end{array}$

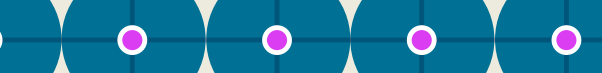

0 0

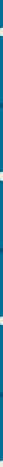

